(c) 2004 International Press

Adv. Theor. Math. Phys. 8 (2004) 217-317

\title{
Supersymmetric Kaluza-Klein reductions of AdS backgrounds
}

\author{
School of Mathematics, The University of Edinburgh, United Kingdom \\ j.m.figueroa@ed.ac.uk
}

\section{Joan Simón}

The Weizmann Institute of Physical Sciences, Rehovot, Israel

Department of Physics and Astronomy, University of Pennsylvania, United States

Kavli Institute of Theoretical Physics, University of California, Santa

Barbara, United States

jsimon@bokchoy.hep.upenn.edu

\begin{abstract}
This paper contains a classification of smooth Kaluza-Klein reductions (by one-parameter subgroups) of the maximally supersymmetric anti de Sitter backgrounds of supergravity theories. We present a classification of one-parameter subgroups of isometries of anti de Sitter spaces, discuss the causal properties of their orbits on these manifolds, and discuss their action on the space of Killing spinors. We analyse the problem of which quotients admit a spin structure. We then apply these results to write down the list of smooth everywhere spacelike supersymmetric quotients of $\mathrm{AdS}_{3} \times S^{3}\left(\times \mathbb{R}^{4}\right), \mathrm{AdS}_{4} \times S^{7}, \mathrm{AdS}_{5} \times S^{5}$ and $\mathrm{AdS}_{7} \times S^{4}$, and the fraction of supersymmetry preserved by each quotient. The results are summarised in tables which should be useful on their own. The paper also includes a discussion of supersymmetry of singular quotients.
\end{abstract}

e-print archive: http://lanl.arXiv.org/abs/hep-th/0401206 


\section{Introduction and motivation}

In [1] we started a programme of classification of quotients of supersymmetric backgrounds of M-theory, of which this paper is part. Our initial motivation was the exploration of the then novel sectors of type II string theory which emerged by the embedding of the Melvin universe [2] into string theory $[3,4,5,6,7,8,9,10,11,12,13,14,15,16,17]$. These are the so-called fluxbranes. Their supergravity description is in terms of the Kaluza-Klein reduction of eleven-dimensional Minkowski spacetime along the orbits of suitable one-parameter subgroups of the Poincaré group, and in [1] we classified all such reductions leading to a smooth quotient and, in particular, we identified those for which the quotient was supersymmetric. These we christened generalised supersymmetric fluxbranes. One such fluxbrane, the nullbrane, is obtained by quotienting by a combination of a translation and a null rotation and such a quotient, when discretised, gives rise to an exact time-dependent background of string theory [18, 19, 20, 21, 22] describing a regular stable supersymmetric cosmology interpolating smoothly between a big crunch phase and a big bang phase. It can be understood as a desingularisation of the parabolic orbifold of [23].

We continued our programme by classifying the supersymmetric KaluzaKlein reductions of the elementary half-BPS backgrounds of M-theory: the M2- and M5-branes [24] and the gravitational wave and the Kaluza-Klein monopole [25]. This yielded a number of novel string backgrounds of type II string theory which can be interpreted as intersections of type II branes with generalised fluxbranes. It also yielded two other classes of backgrounds without a clear physical interpretation. The first such class, which were termed 'exotic' in [24, 25], can be shown to suffer from causal pathologies such as closed lightlike and timelike curves [26], despite being obtained as quotients by a freely acting, everywhere spacelike Killing vector. The second class involves the action of a 'null translation' along the brane worldvolume plus rotations in transverse planes. Even though these are free from closed causal curves, their interpretation remains an open question. Crucial to this analysis is the asymptotic flatness of the spacetimes, which also holds in the case of intersecting branes, to which the analysis can be extended [27].

In this work, we shall extend this programme to the Kaluza-Klein reductions of Freund-Rubin vacua of the form $\operatorname{AdS}_{p+1} \times S^{q}$. These vacua can be understood as near-horizon geometries of the asymptotically flat brane backgrounds, and in this limit there is a symmetry enhancement giving rise to an increased number of allowed quotients. Some of these quotients are also allowed in the brane backgrounds $[24,25]$ themselves and can be thus 
interpreted as near-horizon geometries of the intersection of branes with fluxbranes and nullbranes.

Using the AdS/CFT correspondence [28], D-branes in fluxbrane backgrounds provide a connection with non-commutative (and non-local) gauge theories, the so-called dipole theories [29, 30, 31, 32, 33], whereas D-branes in the nullbrane vacuum [34] (or their dual descriptions) provide explicit time-dependent backgrounds in string theory. See $[35,36]$ for field theory dual proposals. Other interesting quotients are concerned with the possible existence of higher dimensional analogues of the family of BTZ black holes in asymptotically $\mathrm{AdS}_{3}$ spacetimes $[37,38]$. It is interesting to understand the geometry of these new quotients as a first step to extending the AdS/CFT correspondence to lorentzian orbifolds of AdS, in particular, in the context of time dependent backgrounds [36], as was done for the selfdual orbifolds [39] in [40].

The main result of this paper is then a classification of the smooth supersymmetric quotients of Freund-Rubin backgrounds of AdS type by oneparameter subgroups of isometries. This work, together with [41], completes some of the partial results in the literature concerning the geometry of quotients of $\operatorname{AdS}[36,42,43,44,45,46,47,48,49,50]$. Using the techniques developed in [41], it is straightforward to derive the explicit geometries of the corresponding Kaluza-Klein backgrounds associated with the one-parameter subgroups studied in the present paper. We choose not to do this in the present paper, but may return to it elsewhere.

At a purely technical level, the classification problem in this paper is solved by reducing to that of flat background, as was done in $[24,25,27]$, with one crucial difference. In the latter case the flat space in question is the asymptotic spacetime of the brane background and hence we could bring to bear the results of [1]. Here the flat spaces are $\mathbb{R}^{2, p}$ and $\mathbb{R}^{q+1}$ into which we can embed $\mathrm{AdS}_{p+1}$ (locally) and $S^{q}$ as quadrics, and this makes the classification problem more complicated as it is necessary to classify adjoint orbits of $\mathfrak{s o}(2, p)$. This has been done by Boubel [51] as a special case of a more general problem. Boubel's method is thus perhaps unnecessarily complex for the case at hand and partial results are obtained in [38] for $p=2$ and [52] for $p=3$. An elementary derivation for general $p$ appears in a recent paper of Madden and Ross [53]. We will base our classification on a refinement of these results.

Having obtained the possible quotients, the issue of supersymmetry is easily dealt with by exploiting the one-to-one equivariant correspondence between Killing spinors on $\mathrm{AdS}_{p+1} \times S^{q}$ and parallel spinors in a flat space, 
here $\mathbb{R}^{2, p} \times \mathbb{R}^{q+1}$. This follows from Bär's cone construction [54] and its lorentzian extension [55] as will be explained in a more general context in a forthcoming paper [56] devoted to a classification of supersymmetric FreundRubin backgrounds.

This paper is organised as follows. In Section 2 we set up the problem of classifying one-parameter groups of isometries of anti-de Sitter spaces. This is equivalent to the determination of the orbits in (the projectivisation of) the Lie algebra $\mathfrak{s o}(2, p)$ under the action of the adjoint group $\mathrm{SO}(2, p)$. This is in turn a special case of the problem of determining the normal forms of skew-symmetric linear transformations in a pseudo-euclidean space, which has been solved recently by Boubel [51], whose results we use. Each normal form is a direct sum of a finite number (with parameters) of elementary blocks, whose enumeration is the purpose of Section 3. Great care has been exercised in distinguishing between blocks which are related by an orientation-reversing transformation. Our results therefore constitute a (necessary) refinement of the results of [51] and of [53], and on the results of $[38,52]$ for low-dimensional anti-de Sitter spaces. The elementary blocks are displayed matricially in Tables 1,2 and 3 , and as elements of $\mathfrak{s o}(2, p)$ in Table 4. In Section 4 we classify the orbits of $\mathfrak{s o}(2, p)$ under $\mathrm{SO}(2, p)$ for the cases of interest $p=2,3,4,6$. For each such $p$, we determine the corresponding Killing vectors acting on $\mathrm{AdS}_{p+1}$ and determine their causal character on $\mathrm{AdS}_{p+1}$, paying close attention to those Killing vectors whose norm in $\mathrm{AdS}_{p+1}$ is bounded below, as only such vectors can give rise to quotients of the Freund-Rubin backgrounds of the type $\operatorname{AdS}_{p+1} \times S^{q}$ which are free from causal pathologies. At the end of this section we will have an enumeration of possible Killing vectors. We have been careful in enumerating them consistently with the embedding $\mathfrak{s o}(2, p) \subset \mathfrak{s o}(2, p+1)$ or equivalently with the foliation of $\mathrm{AdS}_{p+2}$ by $\mathrm{AdS}_{p+1}$ leaves. In Section 5 we analyse the issue of what happens to supersymmetry under quotients for Freund-Rubin backgrounds of the form $\mathrm{AdS}_{p+1} \times S^{q}$. This is done from the point of view of supergravity; although we do comment on the phenomenon of 'supersymmetry without supersymmetry' which illustrates the difference between supersymmetry in supergravity and in M-theory. We first derive a criterion for the existence of a spin structure in the quotient which reduces to a simple calculation in a Clifford algebra. We also summarise the representation-theoretical approach of [57] to determining the Killing spinors of an AdS background (summarised in Table 5 for the backgrounds of interest) in order to set up the problem of determining the supersymmetry preserved under a quotient. In Section 6 we apply the preceding technology to classify the smooth spacelike supersymmetric quotients of the Freund-Rubin backgrounds of several supergravity theories. In Section 6.1 we tackle the Freund-Rubin $\mathrm{AdS}_{3} \times S^{3}$ vacuum of six-dimensional $(1,0)$ and 
$(2,0)$ supergravities and (primarily) its half-BPS lift $\mathrm{AdS}_{3} \times S^{3} \times \mathbb{R}^{4}$ to IIB supergravity. In Sections 6.2, 6.3 and 6.4 we do the same for the FreundRubin vacua of eleven-dimensional and IIB supergravities. These results are summarised in Tables 6, 7, 8 and 9. For the benefit of the impatient reader, we summarise the notation in those tables as follows:

- $\boldsymbol{e}_{i j}=\boldsymbol{e}_{i} \wedge \boldsymbol{e}_{j} \in \Lambda^{2} \mathbb{R}^{2, p} \cong \mathfrak{s o}(2, p)$ with $\boldsymbol{e}_{1}$ and $\boldsymbol{e}_{2}$ timelike and the rest spacelike; and

- $R_{i j} \in \mathfrak{s o}(q+1)$ is the infinitesimal generator of rotations in the $(i j)$ plane in $\mathbb{R}^{q+1}$.

In this way it should be possible to use our results without the time-consumingalbeit ultimately rewarding - task of reading the rest of the paper.

The paper concludes in Section 7 with a discussion of supersymmetry for singular quotients. This section is not as detailed as the rest of the paper, and in it we simply state generic conditions which allow the preservation of supersymmetry in a quotient that is not necessarily smooth. The results are again summarised in tabular form.

\section{One-parameter subgroups of isometries of $\mathrm{AdS}_{p+1}$}

In this section we set up the classification problem of one-parameter subgroups of isometries of $\mathrm{AdS}_{p+1}$ and outline our strategy to solve it.

For the purposes of this paper, $\mathrm{AdS}_{p+1}$ shall denote a simply-connected lorentzian spaceform with negative constant sectional curvature. Equivalently, it is the universal covering space of the quadric

$$
-\left(t_{1}\right)^{2}-\left(t_{2}\right)^{2}+\left(x_{1}\right)^{2}+\cdots+\left(x_{p}\right)^{2}=-R^{2}
$$

in $\mathbb{R}^{2, p}=\left\{\left(t_{1}, t_{2}, x_{1}, \ldots, x_{p}\right)\right\}$. Strictly speaking, this is $\mathrm{AdS}_{p+1}$ with radius of curvature $R$, where $R$ can be any positive number. The quadric has closed timelike curves; for example,

$$
t \mapsto(R \cos t, R \sin t, 0, \ldots, 0)
$$

and in the universal covering space these curves are unwrapped. The isometry group of the quadric is $\mathrm{O}(2, p)$, which acts linearly on $\mathbb{R}^{2, p}$ and preserves the quadric. In the Freund-Rubin supergravity backgrounds which are the focus of this work, the AdS factor contains more information than 
just the metric: it also has an orientation, hence the true symmetry group of the quadric is $\mathrm{SO}(2, p)$. This, however, is not the isometry group of the simply-connected $\mathrm{AdS}_{p+1}$. The reason is simple. The group $\mathrm{SO}(2, p)$ has a maximal connected compact subgroup $\mathrm{SO}(2) \times \mathrm{SO}(p)$. The $\mathrm{SO}(2)$ factor is generated by the Killing vector $t_{1} \frac{\partial}{\partial t_{2}}-t_{2} \frac{\partial}{\partial t_{1}}$, of which the closed timelike curves above are orbits. However in $\mathrm{AdS}_{p+1}$ these curves are not closed, hence this Killing vector does not generate a circle subgroup but an $\mathbb{R}$ subgroup. Indeed, the symmetry group of $\mathrm{AdS}_{p+1}$ is actually the infinite cover $\widetilde{\mathrm{SO}}(2, p)$ of $\mathrm{SO}(2, p)$, in which the above $\mathrm{SO}(2)$ subgroup is unwrapped to an $\mathbb{R}$-subgroup. Of course, both $\mathrm{SO}(2, p)$ and $\widetilde{\mathrm{SO}}(2, p)$ share the same Lie algebra $\mathfrak{s o}(2, p)$ and, furthermore, their adjoint actions agree, since $\widetilde{\mathrm{SO}}(2, p)$ is a central extension of $\mathrm{SO}(2, p)$ (see the discussion in Section 5.1.2) and the adjoint representation is trivial for the centre, whence the action factors to $\mathrm{SO}(2, p)$.

Every one-parameter subgroup of isometries is generated by some element in the Lie algebra of isometries. Indeed, one-parameter subgroups of a Lie group $G$ are in one-to-one correspondence with the projectivised Lie algebra $\mathbb{P} \mathfrak{g}$. In other words, two elements $X$ and $Y$ of $\mathfrak{g}$ generate the same subgroup if they are collinear. Moreover, when $G$ acts by isometries on some space, we will also identify one-parameter groups which are related by conjugation in $G$ (or more generally by conjugation by the isometry group of the space in question, which may be larger than $G$ ) since the corresponding orbits will be isometric. This means that we are interested in classifying the equivalence classes of elements $X \in \mathfrak{g}$ under

$$
X \sim t g X g^{-1} \quad \text { where } t \in \mathbb{R}^{\times} \text {and } g \in G .
$$

In the case of interest, where $G=\widetilde{\mathrm{SO}}(2, p)$, it will actually be enough to classify the equivalence classes of elements $X \in \mathfrak{s o}(2, p)$ under (2.2) with $G=\mathrm{SO}(2, p)$. There are many partial results in the literature on this type of problem, culminating more or less with the work of Boubel [51].

Boubel has determined the normal forms for a (skew-)symmetric endomorphism $B$ relative to a nondegenerate (skew-)symmetric bilinear form $A$ in a $\mathbb{K}$-vector space. We are interested in the case where $\mathbb{K}=\mathbb{R}, A$ is symmetric and $B$ is skew-symmetric. Moreover we are interested in the case where $A$ has signature $(2, p)$. These normal forms can be decomposed into elementary blocks to which $A$ and $B$ restrict, with $A$ still nondegenerate. As a result the blocks have signature $(m, n)$ where $m \leq 2$ and $n \leq p$. The tables of Boubel (as reproduced by Neukirchner [58] and Leitner [59]) classify these building blocks up to the action of the orthogonal group of $A$; but in this analysis the orientation plays no role and as a result, for the present 
purposes, the classification contains discrete ambiguities which we will have to resolve.

Boubel departs from the observation, as did others who have also looked into this problem $[38,52]$, that if $\lambda \in \mathbb{C}$ is an eigenvalue of $B$, then so are $-\lambda, \bar{\lambda}$ and $-\bar{\lambda}$. Several cases are therefore possible, which can be labelled by the minimal polynomial $\mu(x)$ of $B$ :

1. $\lambda=0$, with $\mu(x)=x^{n}$;

2. $\lambda=\beta \in \mathbb{R}^{\times}$, with $\mu(x)=\left(x^{2}-\beta^{2}\right)^{n}$;

3. $\lambda=i \varphi \in i \mathbb{R}^{\times}$, with $\mu(x)=\left(x^{2}+\varphi^{2}\right)^{n}$; and

4. $\lambda=\beta+i \varphi \in \mathbb{C} \backslash(\mathbb{R} \cup i \mathbb{R})$, with $\mu(x)=\left(\left(x^{2}+\beta^{2}+\varphi^{2}\right)^{2}-4 \beta^{2} x^{2}\right)^{n}$.

To each of these minimal polynomials there are associated (real) Jordan normal forms. The actual form depends on the dimension of the block, which is a positive integer multiple $k$ of the index of nilpotency $n$ in the above expressions. Let $I_{k}$ denote the rank $k$ identity matrix.

The normal form with minimal polynomial $\mu(x)=x^{n}$ and characteristic polynomial $\chi(x)=x^{n k}$ is the $n k \times n k$ matrix:

$$
\left(\begin{array}{ccc}
0 & I_{k} & \\
& \ddots & I_{k} \\
& & 0
\end{array}\right),
$$

whereas the one with minimal polynomial $\mu(x)=\left(x^{2}-\beta^{2}\right)^{n}$ and characteristic polynomial $\chi(x)=\mu(x)^{k}$ is the $2 n k \times 2 n k$ matrix:

$$
\left(\begin{array}{ccc}
M_{2 k} & I_{2 k} & \\
& \ddots & I_{2 k} \\
& & M_{2 k}
\end{array}\right),
$$

where $M_{2 k}$ is the diagonal $2 k \times 2 k$ matrix with alternating entries $+\beta$ and $-\beta$.

Similarly, the normal form with minimal polynomial $\mu(x)=\left(x^{2}+\varphi^{2}\right)^{n}$ and characteristic polynomial $\chi(x)=\mu(x)^{k}$ is the $2 k n \times 2 k n$ matrix:

$$
\left(\begin{array}{ccc}
J_{2 k} & I_{2 k} & \\
& \ddots & I_{2 k} \\
& & J_{2 k}
\end{array}\right)
$$


where $J_{2 k}$ is the block-diagonal $2 k \times 2 k$ matrix consisting of $k$ equal $2 \times 2$ blocks consisting of the matrix $\left(\begin{array}{cc}0 & -\varphi \\ \varphi & 0\end{array}\right)$.

Finally the normal form with minimal polynomial $\mu(x)=\left(\left(x^{2}+\beta^{2}+\right.\right.$ $\left.\left.\varphi^{2}\right)^{2}-4 \beta^{2} x^{2}\right)^{n}$ and characteristic polynomial $\chi(x)=\mu(x)^{k}$ is the $4 n k \times 4 n k$ matrix:

$$
\left(\begin{array}{ccc}
L_{4 k} & I_{4 k} & \\
& \ddots & I_{4 k} \\
& & L_{4 k}
\end{array}\right)
$$

where $L_{4 k}(\beta, \varphi)$ is the block-diagonal $4 k \times 4 k$ matrix consisting of $k$ equal $4 \times 4$ blocks consisting of the matrix

$$
\left(\begin{array}{cccc}
\beta & -\varphi & & \\
\varphi & \beta & & \\
& & -\beta & \varphi \\
& & -\varphi & -\beta
\end{array}\right)
$$

The fact that $B$ is skew-symmetric implies conditions on the entries of the (nondegenerate) inner-product of the elements of the Jordan basis. One can then argue that by changing basis in such a way that the normal form remains unchanged, one can set to zero all inner products which are not constrained by the Jordan form of $B$. It is then a simple matter, albeit a little tedious, to select the possible normal forms with signature $(m, n)$ for $m \leq 2$. These blocks are tabulated in [51, 58, 59] and an elementary derivation can be found in [53]. Boubel's method is equivalent (for the case under consideration) to the one employed in $[38,52,53]$.

From the supergravity point of view, they all share the drawback that little attention is paid to whether one conjugates by $\mathrm{SO}(2, p)$ or $\mathrm{O}(2, p)$. We will therefore be forced to refine these results. This is done simply by taking each normal form in turn and investigating whether the effect of an orientation-reversing orthogonal transformation can be undone by an orientation-preserving orthogonal transformation. If this is not possible, then there are two normal forms up to the action of the special orthogonal group, whereas if it is possible then there is a single normal form. In the following section we summarise the results of these investigations. The calculations are routine. 


\section{The elementary blocks}

Let $\mathbb{V}=\mathbb{R}^{2, p}$ and $B$ be a skew-symmetric endomorphism of $\mathbb{V}$. Associated with $B$ there will be an orthogonal decomposition $\mathbb{V}=\mathbb{V}_{1} \oplus \cdots \oplus \mathbb{V}_{k}$ into nondegenerate subspaces stabilised by $B$. Moreover we can assume that the $\mathbb{V}_{k}$ are indecomposable, so that the restriction of $B$ to any of the $\mathbb{V}_{k}$ does not break down into further nondegenerate blocks. In this way every endomorphism $B$ will decompose into elementary blocks $B_{k}$, namely their restrictions to each of the $\mathbb{V}_{k}$, and conversely, from a knowledge of all the possible blocks, we can assemble all the possible endomorphisms $B$. We are interested in the normal forms of $B$ up to orientation-preserving isometries of $\mathbb{R}^{2, p}$; that is, up to the action of $\mathrm{SO}(2, p)$ and, as we will see, this requires knowing the normal forms of the elementary blocks of signature $(m, n)$ with $m \leq 2$ and $n \leq p$ up to the action of both $\mathrm{O}(m, n)$ and $\mathrm{SO}(m, n)$. We now describe the elementary blocks which are summarised in Tables 1, 2 and 3.

There are two possible trivial blocks of size 1, corresponding to signatures $(0,1)$ and $(1,0)$. Recall that the $\mathbb{V}_{k}$ are nondegenerate, hence if $B$ leaves invariant a null direction then this signals the existence of a larger elementary block - in fact, of dimension at least 3 . These two blocks are denoted $B^{(0,1)}$ and $B^{(1,0)}$, respectively.

There are three possible blocks of size 2, corresponding to signatures $(0,2),(1,1)$ and $(2,0)$. The normal forms are well-known, corresponding to a rotation in the case of definite signature or a boost in the case of signature $(1,1)$. We will follow the mnemonic convention that boost parameters will be denoted $\beta$ and rotation parameters (i.e., angles) will be denoted $\varphi$. These blocks are thus denoted $B^{(0,2)}(\varphi), B^{(1,1)}(\beta)$ and $B^{(2,0)}(\varphi)$, respectively, with the understanding that these parameters are never zero. We may often belabour the point by writing $B^{(0,2)}(\varphi \neq 0)$, etc. Let us now write these blocks explicitly. We will always write them relative to a pseudo-orthonormal basis ordered in such a way that the timelike directions appear first. We will refer to such a basis as an ordered frame. Hence relative to an ordered frame, the metric of a $(1,1)$ block is diagonal with entries $(-1,1)$, for a $(2,0)$ block it is diagonal with entries $(-1,-1)$ and for a $(0,2)$ block the diagonal entries are $(1,1)$. Notice that ordered frames are in general not compatible with the Jordan normal form of the endomorphism.

Rather than writing the endomorphisms we prefer to write the corresponding skew-symmetric bilinear forms; that is, the corresponding elements of $\mathfrak{s o}(m, n)$. To be precise, let $\left\{\boldsymbol{e}_{i}\right\}$ be an ordered frame. Let 
$B \boldsymbol{e}_{j}=\sum_{k} \boldsymbol{e}_{k} B^{k}$. Then the bilinear form associated to $B$ has entries

$$
B_{i j}=\left\langle\boldsymbol{e}_{i}, B \boldsymbol{e}_{j}\right\rangle=\sum_{k} \eta_{i k} B_{j}^{k}
$$

where $\langle-,-\rangle$ denotes the inner product and $\eta_{i k}=\left\langle\boldsymbol{e}_{i}, \boldsymbol{e}_{k}\right\rangle$ its components relative the frame. Recapitulating, then, $B^{(m, n)}$, with or without parameters, will label an element of $\mathfrak{s o}(m, n)$ which, under the vector space isomorphism $\mathfrak{s o}(m, n) \cong \Lambda^{2} \mathbb{R}^{m, n}$, can be represented as a skew-symmetric bilinear form. With these preliminaries we can now arrive at the first few entries in Table 1.

There are three possible three-dimensional signatures $(0,3),(1,2)$ and $(2,1)$ for a subspace of $\mathbb{R}^{2, p}$. Any skew-symmetric endomorphism in a threedimensional space will leave a direction invariant; if this direction is timelike or spacelike then it induces an orthogonal decomposition into smaller blocks; so we only have to consider the case of the direction being null, which can only happen in signatures $(1,2)$ or $(2,1)$. In terms of Lorentz transformations, such an endomorphism corresponds to a null rotation. These are easier to write down in a light-cone basis, but for uniformity we change basis to an ordered frame and arrive at the last two entries in Table 1.

There are three possible four-dimensional signatures for nondegenerate subspaces of $\mathbb{R}^{2, p}$, namely $(0,4),(1,3)$ and $(2,2)$. Clearly the first two break up into smaller blocks; so we only have to consider signature $(2,2)$, where our lorentzian intuition begins to be challenged. One possibility is a combination of a simultaneous boost in two orthogonal $(1,1)$-planes and a simultaneous rotation in two orthogonal $(2,0)$ - and $(0,2)$-planes. This gives rise to the blocks denoted $B_{ \pm}^{(2,2)}(\beta, \varphi)$ in Table 2 , where the parameters $\beta$ and $\varphi$ can both be chosen to be positive. The blocks denoted $B_{ \pm}^{(2,2)}$ are truly new to this signature and cannot be described in terms of Lorentz transformations: they describe transformations which relate two orthogonal null directions and hence they can first appear in signature $(2,2)$. The blocks $B_{ \pm}^{(2,2)}(\beta)$ consist of a deformation of $B_{ \pm}^{(2,2)}$ by a simultaneous boost in two orthogonal $(1,1)$ planes each containing one of the null directions in $B_{ \pm}^{(2,2)}$. Similarly the blocks $B_{ \pm}^{(2,2)}(\varphi)$ consist of a deformation of $B_{ \pm}^{(2,2)}$ by a simultaneous rotation in a $(2,0)$-plane and in an orthogonal $(0,2)$-plane.

There are three possible signatures for five-dimensional nondegenerate subspaces of $\mathbb{R}^{2, p}:(0,5),(1,4)$ and $(2,3)$. In the euclidean and lorentzian cases $B$ always stabilises a nondegenerate subspace, whence it decomposes into smaller blocks. There is only one possibility left, which is a $(2,3)$ block. There is only such possible indecomposable block up to $\mathrm{SO}(2,3)$ or indeed $\mathrm{O}(2,3)$, which we denote $B^{(2,3)}$ and appears in Table 3 . This corresponds to 


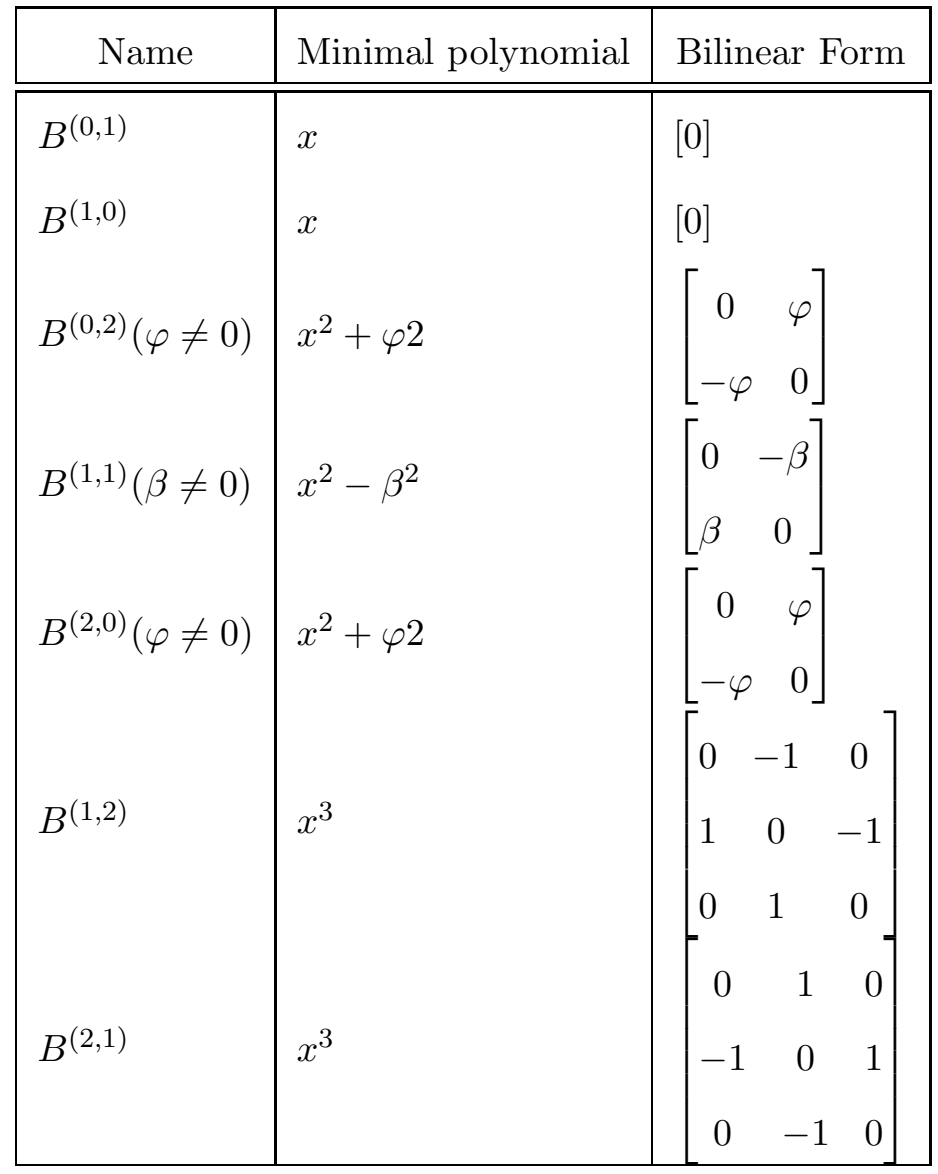

Table 1: Elementary blocks of size $\leq 3$ relative to an ordered frame.

a transformation of the type $B_{ \pm}^{(2,2)}$ together with a null rotation involving a conjugate null direction to one of the ones in $B_{ \pm}^{(2,2)}$ and a third spacelike direction.

Finally, there are three possible signatures for six-dimensional nondegenerate subspaces of $\mathbb{R}^{2, p}:(0,6),(1,5)$ and $(2,4)$. Again for the euclidean and lorentzian cases $B$ always stabilise a nondegenerate subspace, whence it decomposes into smaller blocks. Hence we must only consider the $(2,4)$ blocks. It perhaps comes as a surprise that there is an indecomposable endomorphism in this signature: it is a combination of a simultaneous rotation in each of three orthogonal planes with signature $(0,2),(2,0)$ and $(2,0)$ with a double null rotation in two orthogonal $(1,2)$-planes.

There are no indecomposable blocks of the relevant signatures and of size 


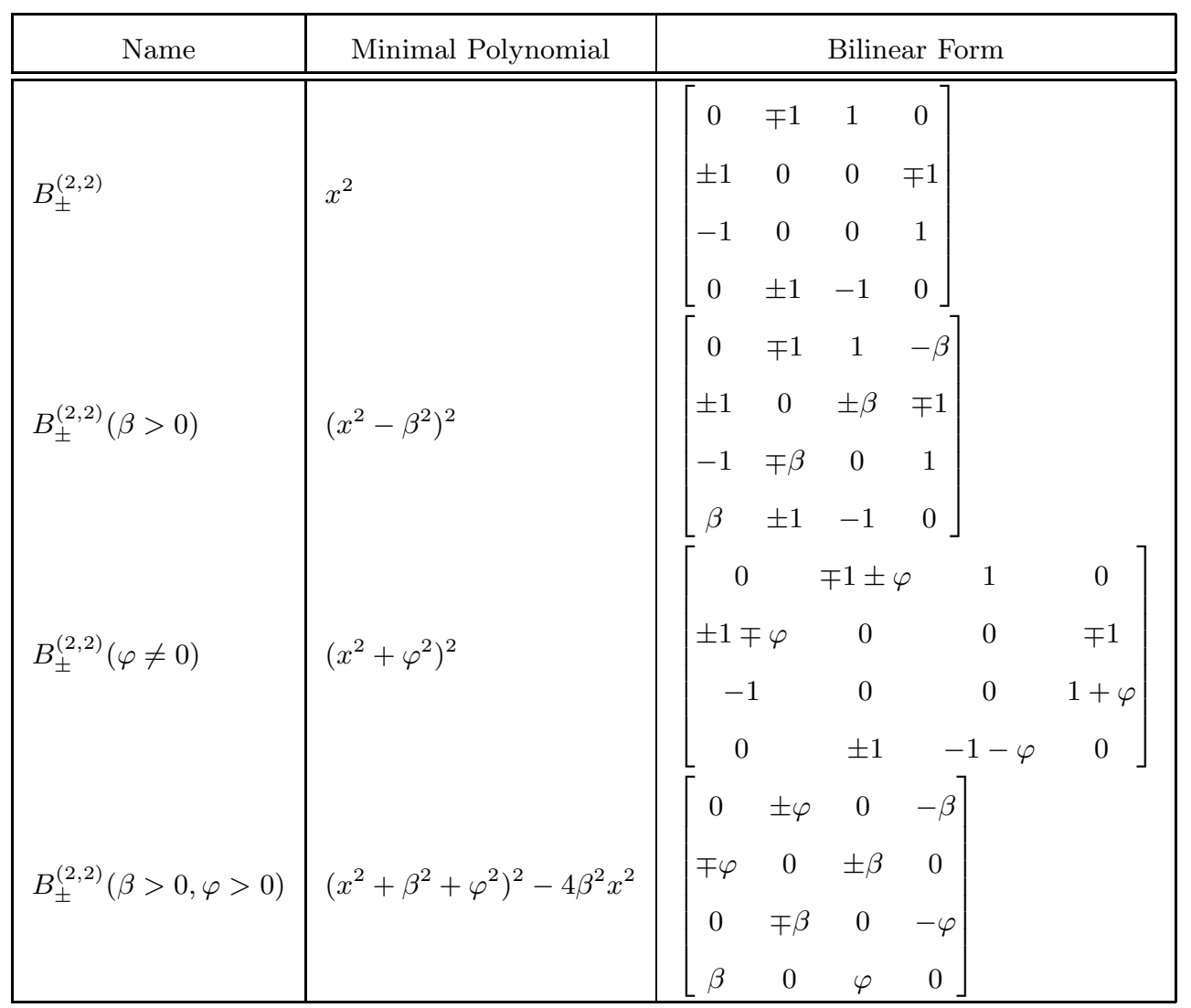

Table 2: Elementary blocks of signature $(2,2)$ relative to an ordered frame.

$>6$.

Finally we must check whether any of the above blocks are related by an orthogonal transformation which does not preserve the orientation. That is, whether two $(m, n)$ blocks which are not $\mathrm{SO}(m, n)$-related are $\mathrm{O}(m, n)$ related. This can only happen when $m+n$ is even, since when $m+n$ is odd, $-S$ preserves orientation whenever $S$ reverses it, yet clearly conjugation by $S$ and $-S$ is the same; whence if two endomorphisms are conjugate under $\mathrm{O}(m, n)$ they are also conjugate under $\mathrm{SO}(m, n)$. When $m+n$ is even, it is easy to investigate how the above blocks change under an orientationreversing orthogonal transformation. One finds the following relations:

- $B^{(0,2)}(\varphi) \sim B^{(0,2)}(-\varphi)$

- $B^{(1,1)}(\beta) \sim B^{(1,1)}(-\beta)$ 


\begin{tabular}{|c|c|c|c|c|c|c|c|}
\hline Name & Minimal Polynomial & \multicolumn{6}{|c|}{ Bilinear Form } \\
\hline \multirow{4}{*}{$B^{(2,3)}$} & \multirow{5}{*}{$x^{5}$} & 0 & 1 & -1 & 0 & - & \\
\hline & & -1 & 0 & 0 & 1 & & \\
\hline & & 1 & 0 & 0 & -1 & & \\
\hline & & 0 & -1 & 1 & 0 & - & \\
\hline \multirow{7}{*}{$B_{ \pm}^{(2,4)}(\varphi \neq 0)$} & & 1 & 0 & 0 & 1 & & \\
\hline & \multirow{6}{*}{$\left(x^{2}+\varphi^{2}\right)^{3}$} & 0 & $\mp \varphi$ & 0 & 0 & & 0 \\
\hline & & $\pm \varphi$ & 0 & 0 & 0 & & $\mp 1$ \\
\hline & & 0 & 0 & 0 & $\varphi$ & & 0 \\
\hline & & 0 & 0 & $-\varphi$ & 0 & & -1 \\
\hline & & 1 & 0 & 1 & 0 & & $\varphi$ \\
\hline & & 0 & \pm 1 & 0 & 1 & & 0 \\
\hline
\end{tabular}

Table 3: Elementary blocks of sizes 5 and 6 relative to an ordered frame.

- $B^{(2,0)}(\varphi) \sim B^{(2,0)}(-\varphi)$

- $B_{+}^{(2,2)} \sim B_{-}^{(2,2)}$

- $B_{+}^{(2,2)}(\varphi) \sim B_{-}^{(2,2)}(\varphi)$

- $B_{+}^{(2,2)}(\beta) \sim B_{-}^{(2,2)}(\beta)$

- $B_{+}^{(2,2)}(\beta, \varphi) \sim B_{-}^{(2,2)}(\beta, \varphi)$, and

- $B_{+}^{(2,4)}(\varphi) \sim B_{-}^{(2,4)}(\varphi)$.

In summary, ignoring orientation, we may ignore the \pm sign in the blocks $B_{ \pm}^{(m, n)}$ with or without parameters, whereas for blocks with parameters but without \pm signs, we may take the parameters to be positive.

It is convenient to write the bilinear forms appearing in each of the elementary blocks in terms of the usual basis for $\Lambda^{2} \mathbb{R}^{m, n} \cong \mathfrak{s o}(m, n)$ consisting of wedge products of the elements of the ordered frame. This information is displayed in Table 4 where we employ the following notation : $\boldsymbol{e}_{1}, \boldsymbol{e}_{2}$ will 
denote the timelike elements of an ordered frame and $\boldsymbol{e}_{3}, \ldots$ will denote the spacelike elements. In general, the two-form is given by $\frac{1}{2} \sum_{i, j} B^{i j} \boldsymbol{e}_{i} \wedge \boldsymbol{e}_{j}$, where $B^{i j}$ is obtained by raising the indices of $B_{i j}$ with the metric. This explains the apparent discrepancies in signs from some of the entries in Tables 1, 2, 3 and in Table 4.

\begin{tabular}{|l|l|}
\hline \multicolumn{1}{|c|}{ Name } & \multicolumn{1}{c|}{ Two-Form } \\
\hline \hline$B^{(0,1)}$ & 0 \\
$B^{(1,0)}$ & 0 \\
$B^{(0,2)}(\varphi \neq 0)$ & $\varphi \boldsymbol{e}_{34}$ \\
$B^{(1,1)}(\beta \neq 0)$ & $\beta \boldsymbol{e}_{13}$ \\
$B^{(2,0)}(\varphi \neq 0)$ & $\varphi \boldsymbol{e}_{12}$ \\
$B^{(1,2)}$ & $\boldsymbol{e}_{13}-\boldsymbol{e}_{34}$ \\
$B^{(2,1)}$ & $\boldsymbol{e}_{12}-\boldsymbol{e}_{23}$ \\
$B_{ \pm}^{(2,2)}$ & $\mp \boldsymbol{e}_{12}-\boldsymbol{e}_{13} \pm \boldsymbol{e}_{24}+\boldsymbol{e}_{34}$ \\
$B_{ \pm}^{(2,2)}(\beta>0)$ & $\mp \boldsymbol{e}_{12}-\boldsymbol{e}_{13} \pm \boldsymbol{e}_{24}+\boldsymbol{e}_{34}+\beta\left(\boldsymbol{e}_{14} \mp \boldsymbol{e}_{23}\right)$ \\
$B_{ \pm}^{(2,2)}(\varphi \neq 0)$ & $\mp \boldsymbol{e}_{12}-\boldsymbol{e}_{13} \pm \boldsymbol{e}_{24}+\boldsymbol{e}_{34}+\varphi\left( \pm \boldsymbol{e}_{12}+\boldsymbol{e}_{34}\right)$ \\
$B_{ \pm}^{(2,2)}(\beta>0, \varphi>0)$ & $\varphi\left( \pm \boldsymbol{e}_{12}-\boldsymbol{e}_{34}\right)+\beta\left(\boldsymbol{e}_{14} \mp \boldsymbol{e}_{23}\right)$ \\
$B^{(2,3)}$ & $\boldsymbol{e}_{12}+\boldsymbol{e}_{13}+\boldsymbol{e}_{15}-\boldsymbol{e}_{24}-\boldsymbol{e}_{34}-\boldsymbol{e}_{45}$ \\
$B_{ \pm}^{(2,4)}(\varphi \neq 0)$ & $\boldsymbol{e}_{15}-\boldsymbol{e}_{35} \pm \boldsymbol{e}_{26}-\boldsymbol{e}_{46}+\varphi\left(\mp \boldsymbol{e}_{12}+\boldsymbol{e}_{34}+\boldsymbol{e}_{56}\right)$ \\
\hline
\end{tabular}

Table 4: The elementary blocks as two-forms.

We are now ready to construct all the one-parameter subgroups of $\mathrm{SO}(2, p)$ for any $p$. We simply play Lego with these building blocks: assembling all the possible $(2, p)$ blocks out of them, taking care to identify the resulting blocks under $\mathrm{SO}(2, p)$, which may require identifying some of the building blocks under $\mathrm{O}(m, n)$. Finally, to obtain the subgroups, we may projectivise the resulting normal forms; although doing so here would be premature because we are ultimately interested in backgrounds of the form $\operatorname{AdS}_{p+1} \times S^{q}$, whence the projectivisation comes after adding a possible element of $\mathfrak{s o}(q+1)$. 


\section{One-parameter subgroups of $\mathrm{SO}(2, p)$}

In this section we will classify the one-parameter subgroups of $\mathrm{SO}(2, p)$ for $p=2,3,4,6$. The general case is no harder in principle, albeit of growing complexity as we will see already in low dimension.

\subsection{One-parameter subgroups of $\mathrm{SO}(2,2)$}

\subsubsection{Adjoint orbits of $\mathfrak{s o}(2,2)$}

The following decompositions are possible of a $(2,2)$-signature space:

- $(2,2)$

- $(2,1) \oplus(0,1)$

- $(1,2) \oplus(1,0)$

- $(2,0) \oplus(0,2)$

- $(2,0) \oplus 2(0,1)$

- $2(1,1)$

- $(1,1) \oplus(1,0) \oplus(0,1)$

- $(0,2) \oplus 2(1,0)$

- $2(0,1) \oplus 2(1,0)$

To each such decomposition there corresponds a block-diagonal decomposition of the endomorphism $B$ and we are instructed to make all possible block-diagonal decompositions and then make sure that no two decompositions can be related by an $\mathrm{SO}(2,2)$ transformation. There is always included in these decompositions the trivial case $B=0$, which in the above list occurs at the end. We will discard this case henceforth. Whenever there is a trivial factor in the decomposition, e.g., $(2,1) \oplus(0,1)$, there is at least one vector $\boldsymbol{v}$ in the kernel of $B$ which is either timelike or spacelike. The orthogonal transformation $\boldsymbol{v} \mapsto-\boldsymbol{v}$ does not change $B$ yet reverses orientation. This means that we can use it to compensate an orientation-reversing change of basis in the nontrivial block(s), here $(2,1)$. In other words, the possible endomorphisms $B$ with a block-diagonal decomposition $(2,1) \oplus(0,1)$ up to the action of $\mathrm{SO}(2,2)$ are in one-to-one correspondence with the possible 
$(2,1)$-blocks up to the action of $\mathrm{O}(2,1)$. We will write this as $(2,1)_{\mathrm{O}}$. More generally, the notation $(m, n)_{\mathrm{O}}$ will denote all possible $(m, n)$-blocks ignoring orientation. Similarly the notation $\left[(m, n) \oplus\left(m^{\prime}, n^{\prime}\right)\right]_{\text {SO }}$ means that we must take into account all possible combinations of $(m, n)$ - and $\left(m^{\prime}, n^{\prime}\right)$-blocks and then identify them by orientation-preserving transformations of the resulting $\left(m+m^{\prime}, n+n^{\prime}\right)$-block which however do not restrict to orientation-preserving transformation in each of the sub-blocks. With this notation, and discarding the trivial case $B=0$, we can write down the possible block-diagonal decompositions of $B$ up to $\mathrm{SO}(2,2)$ :

- $(2,2)$

- $(2,1)_{\mathrm{O}} \oplus(0,1)$

- $(1,2)_{\mathrm{O}} \oplus(1,0)$

- $[(2,0) \oplus(0,2)]_{\mathrm{SO}}$

- $(2,0)_{\mathrm{O}} \oplus 2(0,1)$

- $[2(1,1)]_{\mathrm{SO}}$

- $(1,1)_{\mathrm{O}} \oplus(1,0) \oplus(0,1)$

- $(0,2)_{\mathrm{O}} \oplus 2(1,0)$

We now need to work out the two cases: $[(2,0) \oplus(0,2)]_{\mathrm{SO}}$ and $[2(1,1)]_{\mathrm{SO}}$, since they are not given simply in terms of the elementary blocks we have already classified, but will involve a further restriction of parameters.

For $[(2,0) \oplus(0,2)]_{\text {SO }}$ we have a $(2,2)$-block with bilinear form

$$
\left[\begin{array}{cccc}
0 & \varphi_{1} & 0 & 0 \\
-\varphi_{1} & 0 & 0 & 0 \\
0 & 0 & 0 & \varphi_{2} \\
0 & 0 & -\varphi_{2} & 0
\end{array}\right]
$$

where $\varphi_{1} \varphi_{2} \neq 0$.

Under an $\mathrm{SO}(2,2)$ transformation not in $\mathrm{SO}(2,0) \times \mathrm{SO}(0,2)$ we can change the signs of the $\varphi_{i}$ simultaneously; which allows us to choose $\varphi_{1}>0$, say.

Similarly for $[2(1,1)]_{\text {SO }}$ we have a $(2,2)$-block with bilinear form

$$
\left[\begin{array}{cccc}
0 & 0 & -\beta_{1} & 0 \\
0 & 0 & 0 & -\beta_{2} \\
\beta_{1} & 0 & 0 & 0 \\
0 & \beta_{2} & 0 & 0
\end{array}\right] \quad \text { where } \beta_{1} \beta_{2} \neq 0
$$


which does not look block-diagonal because we had to reorder the basis in order to make the timelike directions appear first. Under $\mathrm{SO}(2,2)$ transformations not in $\mathrm{SO}(1,1) \times \mathrm{SO}(1,1)$ we can exchange $\beta_{1} \leftrightarrow \beta_{2}$ and also change their signs simultaneously. Hence we may choose $\beta_{1} \geq\left|\beta_{2}\right|>0$.

With these considerations behind us, and taking into account Tables 1, 2 and 3 of elementary blocks, we can finally list all the possible elements of $\mathfrak{s o}(2,2)$ modulo the action of $\mathrm{SO}(2,2)$ :

1. $B^{(2,0)}(\varphi>0) \oplus 2 B^{(0,1)}$

2. $B^{(1,1)}(\beta>0) \oplus B^{(1,0)} \oplus B^{(0,1)}$

3. $B^{(2,1)} \oplus B^{(0,1)}$

4. $B^{(0,2)}(\varphi>0) \oplus 2 B^{(1,0)}$

5. $B^{(1,2)} \oplus B^{(1,0)}$

6. $B_{ \pm}^{(2,2)}$

7. $B_{ \pm}^{(2,2)}(\beta>0)$

8. $B_{ \pm}^{(2,2)}(\varphi \neq 0)$

9. $B_{ \pm}^{(2,2)}(\beta>0, \varphi>0)$

10. $B^{(0,2)}\left(\varphi_{1}>0\right) \oplus B^{(2,0)}\left(\varphi_{2} \neq 0\right)$

11. $B^{(1,1)}\left(\beta_{1}\right) \oplus B^{(1,1)}\left(\beta_{2}\right)$, with $\beta_{1} \geq\left|\beta_{2}\right|>0$

We have reordered them to more easily reflect the natural embeddings $\mathfrak{s o}(2,2) \subset$ $\mathfrak{s o}(2,3) \subset \cdots$ later on.

It is convenient to rewrite this list in a more traditional notation. Let $\left\{\boldsymbol{e}_{1}, \ldots, \boldsymbol{e}_{4}\right\}$ be an ordered frame with $\boldsymbol{e}_{1}$ and $\boldsymbol{e}_{2}$ timelike and the rest spacelike. As usual we will write $\boldsymbol{e}_{i j}=\boldsymbol{e}_{i} \wedge \boldsymbol{e}_{j} \in \Lambda^{2} \mathbb{R}^{2,2} \cong \mathfrak{s o}(2,2)$. Then, using Table 4, the above elements can be written as follows:

1. $\varphi \boldsymbol{e}_{12},(\varphi>0)$;

2. $\beta \boldsymbol{e}_{13},(\beta>0)$.

3. $e_{12}-e_{23}$

4. $\varphi \boldsymbol{e}_{34},(\varphi>0)$; 

5. $e_{13}-e_{34}$
6. $\mp e_{12}-e_{13} \pm e_{24}+e_{34}$;
7. $\mp e_{12}-e_{13} \pm e_{24}+e_{34}+\beta\left(e_{14} \mp e_{23}\right),(\beta>0)$;
8. $\mp \boldsymbol{e}_{12}-\boldsymbol{e}_{13} \pm \boldsymbol{e}_{24}+\boldsymbol{e}_{34}+\varphi\left( \pm \boldsymbol{e}_{12}+\boldsymbol{e}_{34}\right),(\varphi \neq 0)$;
9. $\varphi\left( \pm e_{12}-e_{34}\right)+\beta\left(e_{14} \mp e_{23}\right),(\beta>0, \varphi>0)$;
10. $\varphi_{1} \boldsymbol{e}_{12}+\varphi_{2} \boldsymbol{e}_{34},\left(\varphi_{1}>0, \varphi_{2} \neq 0\right)$;
11. $\beta_{1} \boldsymbol{e}_{13}+\beta_{2} \boldsymbol{e}_{24},\left(\beta_{1} \geq\left|\beta_{2}\right|>0\right)$; and

Clearly by letting some of the parameters become 0 , we can subsume some of these cases into others; but we prefer not to do this at this stage. The above list is in one-to-one correspondence with the conjugacy classes of nonzero elements of $\mathfrak{s o}(2,2)$ under $\mathrm{SO}(2,2)$.

A direct comparison with the results of [38] is now possible. We do not think it relevant to perform this comparison in detail here. Let us merely observe that if we were to further identify elements of $\mathfrak{s o}(2,2)$ under the action of $\mathrm{O}(2,2)$, then we would obtain the results of [38] except for the fact that the parameters in their classes $\mathrm{I}_{a}, \mathrm{I}_{b}$ and $\mathrm{I}_{c}$ should then be constrained.

\subsubsection{Causal properties of orbits}

Next we determine the causal properties of the orbits in $\mathrm{AdS}_{3}$ under the oneparameter subgroups. We will do this by computing the norm of the vector field which generates the subgroup. These are easier to compute in the local model for $\mathrm{AdS}_{3}$ given by the quadric in $\mathbb{R}^{2,2}$, since the Killing vectors are the restriction to the quadric of linear vector fields in $\mathbb{R}^{2,2}$. The Killing vectors and also their norms lift to $\mathrm{AdS}_{3}$. This is perhaps most concretely realised by giving coordinates to $\mathrm{AdS}_{3}$ which are adapted to the action of the fundamental group of the quadric and noticing that the Killing vectors (and hence their norms) are invariant under this action, whence they lift trivially. In practical terms, what this means is that if the action of the elementary group is generated by translating a coordinate, $\tau$ say, by a some period $T$, then the local coordinate expressions for the Killing vectors will be periodic in $\tau$ with period $T$. The same will be true, of course, for anti-de Sitter spaces of higher dimension.

We introduce flat coordinates $x^{i}$ for $\mathbb{R}^{2,2}$ associated to the ordered frame $\boldsymbol{e}_{i}$, so that a point in $\mathbb{R}^{2}$ has coordinates $\boldsymbol{x}=\sum x^{i} \boldsymbol{e}_{i}$. In these coordinates 
the metric takes the form

$$
g=\eta_{i j} d x^{i} d x^{j}=-\left(d x^{1}\right)^{2}-\left(d x^{2}\right)^{2}+\left(d x^{3}\right)^{2}+\left(d x^{4}\right)^{2} .
$$

The Killing vector associated to the Lie algebra element $\boldsymbol{e}_{i j} \in \mathfrak{s o}(2,2)$ is $x_{i} \partial_{j}-x_{j} \partial_{i}$, and hence if $X=\frac{1}{2} B^{i j} \boldsymbol{e}_{i j}$, then the norm of the corresponding Killing vector $\xi_{X}=\frac{1}{2} B^{i j}\left(x_{i} \partial_{j}-x_{j} \partial_{i}\right)$ is given by

$$
\left|\xi_{X}\right|^{2}=\eta_{i j} B_{k}^{i} B_{\ell}^{j} x^{k} x^{\ell}=\eta^{i j} B_{i k} x^{k} B_{j \ell} x^{\ell},
$$

which we then restrict to the quadric

$$
-\left(x^{1}\right)^{2}-\left(x^{2}\right)^{2}+\left(x^{3}\right)^{2}+\left(x^{4}\right)^{2}=-R^{2} .
$$

Equation (4.1) is very easily implemented from the explicit expressions of the bilinear forms in Tables 1, 2 and 3: we simply apply the bilinear form as a matrix to the (column) vector $\left(x^{1}, x^{2}, x^{3}, x^{4}\right)=\left(-x_{1},-x_{2}, x_{3}, x_{4}\right)$ and then compute the Minkowski norm of the resulting vector. Doing so, we quickly arrive at the following result for the Killing vectors in the same order as that given above:

1. $|\xi|^{2}=-\varphi^{2}\left(R^{2}+x_{3}^{2}+x_{4}^{2}\right)$, which is unbounded below;

2. $|\xi|^{2}=\beta^{2}\left(R^{2}-x_{2}^{2}+x_{4}^{2}\right)$, which is unbounded below;

3. $|\xi|^{2}=-\left(x_{3}+x_{1}\right)^{2}$, which is unbounded below;

4. $|\xi|^{2}=\varphi^{2}\left(x_{3}^{2}+x_{4}^{2}\right) \geq 0$

5. $|\xi|^{2}=\left(x_{1}+x_{4}\right)^{2} \geq 0$;

6. $|\xi|^{2}=0$;

7. $|\xi|^{2}=\beta^{2} R^{2}+4 \beta\left(x_{1}+x_{4}\right)\left(x_{3} \pm x_{2}\right)$, which is unbounded below;

8. $|\xi|^{2}=-\varphi^{2} R^{2}+2 \varphi\left(\left(x_{1}+x_{4}\right)^{2}+\left(x_{3} \pm x_{2}\right)^{2}\right)$, which is $>-\varphi^{2} R^{2}$ if $\varphi>0$;

9. $|\xi|^{2}=\left(\beta^{2}-\varphi^{2}\right) R^{2}-4 \beta \varphi\left(x_{1} x_{3} \pm x_{2} x_{4}\right)$, which is unbounded below;

10. $|\xi|^{2}=-\varphi_{2}^{2} R^{2}+\left(\varphi_{1}^{2}-\varphi_{2}^{2}\right)\left(x_{3}^{2}+x_{4}^{2}\right)$, which is $\geq-\varphi_{2}^{2} R^{2}$ if $\varphi_{1}^{2} \geq \varphi_{2}^{2}$; and

11. $|\xi|^{2}=\beta_{1}^{2} R^{2}+\left(\beta_{2}^{2}-\beta_{1}^{2}\right)\left(x_{2}^{2}-x_{4}^{2}\right)$, which is positive if $\beta_{1}= \pm \beta_{2}$ and unbounded below otherwise.

Before going on to the next dimension, let us pause a moment to explain how these norms will be used. In future sections, we will be interested in quotients of $\mathrm{AdS}_{p+1} \times S^{q}$ by everywhere spacelike Killing vectors. The sphere 
being compact and riemannian, the norm of a Killing vector is non-negative and bounded above. Moreover if $q$ is odd there are Killing vectors whose norm on the sphere is positive and pinched away from zero. This means that in those cases we can allow Killing vectors whose norms are bounded below on $\mathrm{AdS}_{p+1}$ but not necessarily positive. Such reductions appeared for the first time in [24] in the context of reductions of elementary M-branes and are known to have closed causal curves [26]. The same phenomenon will happen here whenever the norm of the Killing vector acting on $\mathrm{AdS}_{p+1}$ is bounded from below and negative in some regions [41].

Notice that the property of the norm of a Killing vector "not being bounded below' in AdS is hereditary under the embeddings $\mathfrak{s o}(2,2) \subset \mathfrak{s o}(2,3) \subset$ ... Hence if a certain Killing vector cannot be used in $\mathrm{AdS}_{p+1}$ it cannot be used either in a higher-dimensional $\mathrm{AdS}$ space, since $\mathrm{AdS}_{n}$ contains $\mathrm{AdS}_{m}$ subspaces for $m<n$ where the norm can already be arbitrarily negative. On the other hand, the norm of a Killing vector in $\mathrm{AdS}_{p+1}$ which is bounded below may become unbounded upon embedding in a higher-dimensional AdS space.

\subsection{One-parameter subgroups of $\mathrm{SO}(2,3)$}

\subsubsection{Adjoint orbits of $\mathfrak{s o}(2,3)$}

The possible decompositions of a skew-symmetric endomorphism $B$ up to conjugation by $\mathrm{SO}(2,3)$ are as follows:

- $(2,3)$

- $(2,2)_{\mathrm{O}} \oplus(0,1)$

- $[(2,1) \oplus(0,2)]_{\mathrm{SO}}=(2,1) \oplus(0,2)_{\mathrm{O}}$

- $(2,1) \oplus 2(0,1)$

- $[(2,0) \oplus(0,2)]_{\mathrm{O}} \oplus(0,1)$

- $(2,0)_{\mathrm{O}} \oplus 3(0,1)$

- $[(1,2) \oplus(1,1)]_{\mathrm{SO}}=(1,2) \oplus(1,1)_{\mathrm{O}}$

- $(1,2) \oplus(1,0) \oplus(0,1)$

- $[2(1,1)]_{\mathrm{O}} \oplus(0,1)$

- $[(1,1) \oplus(0,2)]_{\mathrm{O}} \oplus(1,0)$ 
- $(1,1)_{\mathrm{O}} \oplus 2(0,1) \oplus(1,0)$

- $(0,2)_{\mathrm{O}} \oplus 2(1,0) \oplus(0,1)$

Here, as before, the notation $[2(m, n)]_{\mathrm{O}}$ denotes all possible pairs of $(m, n)$ blocks identified under transformations of $\mathrm{O}(2 m, 2 n)$ which do not belong to $\mathrm{O}(m, n) \times \mathrm{O}(m, n)$; namely the orthogonal transformation which interchanges the two blocks. Some of the above blocks are not elementary and must be separately investigated. For example, the block $[(2,0) \oplus(0,2)]_{\mathrm{O}}$ is represented by the bilinear form

$$
\left[\begin{array}{cccc}
0 & \varphi_{1} & 0 & 0 \\
-\varphi_{1} & 0 & 0 & 0 \\
0 & 0 & 0 & \varphi_{2} \\
0 & 0 & -\varphi_{2} & 0
\end{array}\right] \quad \text { where } \varphi_{1} \varphi_{2} \neq 0
$$

Under $\mathrm{O}(2,2)$ we can change the signs of $\varphi_{i}$ independently, whence we can choose them both positive. Similarly the block $[2(1,1)]_{\mathrm{O}}$ is represented by the bilinear form

$$
\left[\begin{array}{cccc}
0 & 0 & -\beta_{1} & 0 \\
0 & 0 & 0 & -\beta_{2} \\
\beta_{1} & 0 & 0 & 0 \\
0 & \beta_{2} & 0 & 0
\end{array}\right] \quad \text { where } \beta_{1} \beta_{2} \neq 0
$$

Under $\mathrm{O}(2,2)$ we can interchange $\beta_{1}$ and $\beta_{2}$ and change their signs independently; whence we can always choose $\beta_{1} \geq \beta_{2}>0$. Finally we have $[(1,1) \oplus(0,2)]_{\mathrm{O}}$, which is represented by the bilinear form

$$
\left[\begin{array}{cccc}
0 & -\beta & 0 & 0 \\
\beta & 0 & 0 & 0 \\
0 & 0 & 0 & \varphi \\
0 & 0 & -\varphi & 0
\end{array}\right] \quad \text { where } \beta \varphi \neq 0
$$

Under $\mathrm{O}(2,2)$ we can change the sign of $\beta$ and $\varphi$ independently, whence we can choose them positive.

We can now list all the possible elements of $\mathfrak{s o}(2,3)$ up to the action of $\mathrm{SO}(2,3)$ :

1. $B^{(2,0)}(\varphi>0) \oplus 3 B^{(0,1)}$

2. $B^{(1,1)}(\beta>0) \oplus 2 B^{(0,1)} \oplus B^{(1,0)}$

3. $B^{(2,1)} \oplus 2 B^{(0,1)}$ 
4. $B^{(0,2)}(\varphi>0) \oplus 2 B^{(1,0)} \oplus B^{(0,1)}$

5. $B^{(1,2)} \oplus B^{(1,0)} \oplus B^{(0,1)}$

6. $B_{+}^{(2,2)} \oplus B^{(0,1)}$

7. $B_{+}^{(2,2)}(\beta>0) \oplus B^{(0,1)}$

8. $B_{+}^{(2,2)}(\varphi \neq 0) \oplus B^{(0,1)}$

9. $B_{+}^{(2,2)}(\beta>0, \varphi>0) \oplus B^{(0,1)}$

10. $B^{(2,0)}\left(\varphi_{1}>0\right) \oplus B^{(0,2)}\left(\varphi_{2}>0\right) \oplus B^{(0,1)}$

11. $B^{(1,1)}\left(\beta_{1}\right) \oplus B^{(1,1)}\left(\beta_{2}\right) \oplus B^{(0,1)}$, with $\beta_{1} \geq \beta_{2}>0$

12. $B^{(1,1)}(\beta>0) \oplus B^{(0,2)}(\varphi>0) \oplus B^{(1,0)}$

13. $B^{(2,3)}$

14. $B^{(2,1)} \oplus B^{(0,2)}(\varphi>0)$

15. $B^{(1,2)} \oplus B^{(1,1)}(\beta>0)$

where again we have reordered them in such a way that the numbering makes the embedding $\mathfrak{s o}(2,2) \subset \mathfrak{s o}(2,3)$ manifest; that is, each of the cases (1)-(11) is the embedding in $\mathfrak{s o}(2,3)$ of the corresponding case in $\mathfrak{s o}(2,2)$; except that sometimes the extra freedom in conjugating by the larger group $\mathrm{SO}(2,3)$ results in a further constraint on the parameters.

Again we rewrite this list in a more traditional notation. Let $\left\{\boldsymbol{e}_{1}, \ldots, \boldsymbol{e}_{5}\right\}$ be an ordered frame with $\boldsymbol{e}_{1}$ and $\boldsymbol{e}_{2}$ timelike and the rest spacelike. Then, using Table 4, the above elements can be written as follows:

1. $\varphi \boldsymbol{e}_{12},(\varphi>0)$;

2. $\beta \boldsymbol{e}_{13},(\beta>0)$; and

3. $e_{12}-e_{23}$

4. $\varphi \boldsymbol{e}_{34},(\varphi>0)$.

5. $e_{13}-e_{34}$

6. $-e_{12}-e_{13}+e_{24}+e_{34}$

7. $-\boldsymbol{e}_{12}-\boldsymbol{e}_{13}+\boldsymbol{e}_{24}+\boldsymbol{e}_{34}+\beta\left(\boldsymbol{e}_{14}-\boldsymbol{e}_{23}\right),(\beta>0)$; 

8. $-\boldsymbol{e}_{12}-\boldsymbol{e}_{13}+\boldsymbol{e}_{24}+\boldsymbol{e}_{34}+\varphi\left(\boldsymbol{e}_{12}+\boldsymbol{e}_{34}\right),(\varphi \neq 0)$;
9. $\varphi\left(\boldsymbol{e}_{12}-\boldsymbol{e}_{34}\right)+\beta\left(\boldsymbol{e}_{14}-\boldsymbol{e}_{23}\right),(\beta>0, \varphi>0) ;$
10. $\varphi_{1} \boldsymbol{e}_{12}+\varphi_{2} \boldsymbol{e}_{34},\left(\varphi_{i}>0\right)$;
11. $\beta_{1} \boldsymbol{e}_{13}+\beta_{2} \boldsymbol{e}_{24},\left(\beta_{1} \geq \beta_{2}>0\right)$;
12. $\beta \boldsymbol{e}_{13}+\varphi \boldsymbol{e}_{45},(\beta>0, \varphi>0)$;
13. $e_{12}+e_{13}+e_{15}-e_{24}-e_{34}-e_{45}$
14. $\boldsymbol{e}_{12}-\boldsymbol{e}_{23}+\varphi \boldsymbol{e}_{45},(\varphi>0)$;
15. $\boldsymbol{e}_{13}-\boldsymbol{e}_{34}+\beta \boldsymbol{e}_{25},(\beta>0)$;

Again by letting some of the parameters become 0, we can subsume some of these cases into others; but we prefer not to do this at this stage. The above list is in one-to-one correspondence with the conjugacy classes of nonzero elements of $\mathfrak{s o}(2,3)$ under $\mathrm{SO}(2,3)$.

A direct comparison with the results of [52] is now also possible and as in the case of $\mathfrak{s o}(2,2)$, our results morally agree with those of [52] provided we identify elements under the action of $\mathrm{O}(2,3)$.

\subsubsection{Causal properties of orbits}

We compute the norms of the Killing vectors as we did for $\mathfrak{s o}(2,2)$. Notice that we can read off the norms of the Killing vectors (1)-(11) coming from $\mathfrak{s o}(2,2)$ simply by noticing that whenever $R^{2}$ appears in the calculation of the norms for $\mathrm{AdS}_{3}$ we now have $R^{2}+x_{5}^{2}$. Similarly we can read off the norms of decomposable blocks by adding the norms of each of the blocks, taken care that the coordinates should correspond. These considerations and a simple calculation yield immediately the following norms:

1. $|\xi|^{2}=-\varphi^{2}\left(R^{2}+x_{3}^{2}+x_{4}^{2}+x_{5}^{2}\right)$, which is unbounded below;

2. $|\xi|^{2}=\beta^{2}\left(R^{2}-x_{2}^{2}+x_{4}^{2}+x_{5}^{2}\right)$, which is unbounded below;

3. $|\xi|^{2}=-\left(x_{3}+x_{1}\right)^{2}$, which is unbounded below;

4. $|\xi|^{2}=\varphi^{2}\left(x_{3}^{2}+x_{4}^{2}\right) \geq 0 ;$

5. $|\xi|^{2}=\left(x_{1}+x_{4}\right)^{2} \geq 0$; 
6. $|\xi|^{2}=0$;

7. $|\xi|^{2}=\beta^{2}\left(R^{2}+x_{5}^{2}\right)+4 \beta\left(x_{1}+x_{4}\right)\left(x_{3}+x_{2}\right)$, which is unbounded below;

8. $|\xi|^{2}=-\varphi^{2}\left(R^{2}+x_{5}^{2}\right)+2 \varphi\left(\left(x_{1}+x_{4}\right)^{2}+\left(x_{2}+x_{3}\right)^{2}\right)$, which is unbounded below because there are points in $\mathrm{AdS}_{4}$ with arbitrarily small $\left|x_{1}+x_{4}\right|$ and $\left|x_{2}+x_{3}\right|$ and arbitrarily large $R^{2}+x_{5}^{2}=\left(x_{1}+x_{4}\right)\left(x_{1}-x_{4}\right)+\left(x_{2}+\right.$ $\left.x_{3}\right)\left(x_{2}-x_{3}\right)$;

9. $|\xi|^{2}=\left(\beta^{2}-\varphi^{2}\right)\left(R^{2}+x_{5}^{2}\right)-4 \beta \varphi\left(x_{1} x_{3}+x_{2} x_{4}\right)$, which is unbounded below;

10. $|\xi|^{2}=-\varphi_{2}^{2}\left(R^{2}+x_{5}^{2}\right)+\left(\varphi_{1}^{2}-\varphi_{2}^{2}\right)\left(x_{3}^{2}+x_{4}^{2}\right)$, which is unbounded below since in the subspace of $\mathrm{AdS}_{4}$ where $x_{3}=x_{4}=0,\left|x_{5}\right|$ is not bounded;

11. $|\xi|^{2}=\beta_{1}^{2}\left(R^{2}+x_{5}^{2}\right)+\left(\beta_{2}^{2}-\beta_{1}^{2}\right)\left(x_{2}^{2}-x_{4}^{2}\right)$, which is $\geq \beta_{1}^{2} R^{2}$ if $\beta_{1}=\beta_{2}$ and unbounded below otherwise;

12. $|\xi|^{2}=\beta^{2}\left(x_{1}^{2}-x_{3}^{2}\right)+\varphi^{2}\left(x_{4}^{2}+x_{5}^{2}\right)$, which is unbounded below;

13. $|\xi|^{2}=\left(x_{4}-x_{1}\right)^{2}-4\left(x_{2}+x_{3}\right) x_{5}$, which is unbounded below;

14. $|\xi|^{2}=-\left(x_{1}+x_{3}\right)^{2}+\varphi^{2}\left(x_{4}^{2}+x_{5}^{2}\right)$, which is unbounded below; and

15. $|\xi|^{2}=\left(x_{1}+x_{4}\right)^{2}+\beta^{2}\left(x_{2}^{2}-x_{5}^{2}\right)$, which is also unbounded below.

Since we will be applying these results to the reductions of $\mathrm{AdS}_{4} \times S^{7}$ and $S^{7}$ does possess Killing vectors without zeroes we will be needing those Killing vectors on $\mathrm{AdS}_{4}$ whose norms are bounded below. From the above list we see immediately that these are (4), (5), (6) and (11) for $\beta_{1}=\beta_{2}$.

\subsection{One-parameter subgroups of $\mathrm{SO}(2,4)$}

\subsubsection{Adjoint orbits of $\mathfrak{s o}(2,4)$}

Up to conjugation by $\mathrm{SO}(2,4)$ a skew-symmetric endomorphism $B$ can have the following block-diagonal decompositions:

- $(2,4)$

- $(2,3) \oplus(0,1)$

- $[(2,2) \oplus(0,2)]_{\mathrm{SO}}=(2,2)_{\mathrm{O}} \oplus(0,2)$

- $(2,2)_{\mathrm{O}} \oplus 2(0,1)$ 
- $(2,1) \oplus(0,2)_{\mathrm{O}} \oplus(0,1)$

- $(2,1) \oplus 3(0,1)$

- $2(1,2)$

- $(1,2) \oplus(1,1)_{\mathrm{O}} \oplus(0,1)$

- $(1,2) \oplus(0,2)_{\mathrm{O}} \oplus(1,0)$

- $(1,2) \oplus(1,0) \oplus 2(0,1)$

- $[(2,0) \oplus 2(0,2)]_{\mathrm{SO}}=(2,0) \oplus[2(0,2)]_{\mathrm{O}}$

- $(2,0)_{\mathrm{O}} \oplus(0,2)_{\mathrm{O}} \oplus 2(0,1)$

- $(2,0)_{\mathrm{O}} \oplus 4(0,1)$

- $[2(1,1) \oplus(0,2)]_{\mathrm{SO}}=[2(1,1)]_{\mathrm{O}} \oplus(0,2)$

- $[2(1,1)]_{\mathrm{O}} \oplus 2(0,1)$

- $(1,1)_{\mathrm{O}} \oplus(0,2)_{\mathrm{O}} \oplus(0,1) \oplus(1,0)$

- $(1,1)_{\mathrm{O}} \oplus(1,0) \oplus 3(0,1)$

- $[2(0,2)]_{\mathrm{O}} \oplus 2(1,0)$

- $(0,2)_{\mathrm{O}} \oplus 2(1,0) \oplus 2(0,1)$

We still have to work out $[2(0,2)]_{\mathrm{O}}$ which is represented by a bilinear form of the type

$$
\left[\begin{array}{cccc}
0 & \varphi_{1} & 0 & 0 \\
-\varphi_{1} & 0 & 0 & 0 \\
0 & 0 & 0 & \varphi_{2} \\
0 & 0 & -\varphi_{2} & 0
\end{array}\right] \quad \text { where } \varphi_{1} \varphi_{2} \neq 0
$$

Under $O(2,2)$ we can interchange $\varphi_{1}$ and $\varphi_{2}$ and change their signs independently; whence we choose $\varphi_{1} \geq \varphi_{2}>0$.

With these considerations behind us, we can now list all the possible elements of $\mathfrak{s o}(2,4)$ up to the action of $\mathrm{SO}(2,4)$, where we have again ordered them in such a way that (1)-(15) correspond to the embedding in $\mathfrak{s o}(2,4)$ of the corresponding elements of $\mathfrak{s o}(2,3)$ :

1. $B^{(2,0)}(\varphi>0) \oplus 4 B^{(0,1)}$

2. $B^{(1,1)}(\beta>0) \oplus 3 B^{(0,1)} \oplus B^{(1,0)}$ 
3. $B^{(2,1)} \oplus 3 B^{(0,1)}$

4. $B^{(0,2)}(\varphi>0) \oplus 2 B^{(1,0)} \oplus 2 B^{(0,1)}$.

5. $B^{(1,2)} \oplus 2 B^{(0,1)} \oplus B^{(1,0)}$

6. $B_{+}^{(2,2)} \oplus 2 B^{(0,1)}$

7. $B_{+}^{(2,2)}(\beta>0) \oplus 2 B^{(0,1)}$

8. $B_{+}^{(2,2)}(\varphi \neq 0) \oplus 2 B^{(0,1)}$

9. $B_{+}^{(2,2)}(\beta>0, \varphi>0) \oplus 2 B^{(0,1)}$

10. $B^{(2,0)}\left(\varphi_{1}>0\right) \oplus B^{(0,2)}\left(\varphi_{2}>0\right) \oplus 2 B^{(0,1)}$

11. $B^{(1,1)}\left(\beta_{1}\right) \oplus B^{(1,1)}\left(\beta_{2}\right) \oplus 2 B^{(0,1)}$, with $\beta_{1} \geq \beta_{2}>0$

12. $B^{(1,1)}(\beta>0) \oplus B^{(0,2)}(\varphi>0) \oplus B^{(0,1)} \oplus B^{(1,0)}$

13. $B^{(2,3)} \oplus B^{(0,1)}$

14. $B^{(2,1)} \oplus B^{(0,2)}(\varphi>0) \oplus B^{(0,1)}$

15. $B^{(1,2)} \oplus B^{(1,1)}(\beta>0) \oplus B^{(0,1)}$

16. $B^{(0,2)}\left(\varphi_{1}\right) \oplus B^{(0,2)}\left(\varphi_{2}\right) \oplus 2 B^{(1,0)}$, with $\varphi_{1} \geq \varphi_{2}>0$

17. $B^{(1,2)} \oplus B^{(0,2)}(\varphi>0) \oplus B^{(1,0)}$

18. $B_{ \pm}^{(2,4)}(\varphi \neq 0)$

19. $B_{+}^{(2,2)}(\beta>0) \oplus B^{(0,2)}(\varphi \neq 0)$

20. $B_{+}^{(2,2)}\left(\varphi_{1} \neq 0\right) \oplus B^{(0,2)}\left(\varphi_{2} \neq 0\right)$

21. $B_{+}^{(2,2)} \oplus B^{(0,2)}(\varphi \neq 0)$

22. $B_{+}^{(2,2)}\left(\beta>0, \varphi_{1}>0\right) \oplus B^{(0,2)}\left(\varphi_{2} \neq 0\right)$

23. $2 B^{(1,2)}$

24. $B^{(2,0)}\left(\varphi_{1} \neq 0\right) \oplus B^{(0,2)}\left(\varphi_{2}\right) \oplus B^{(0,2)}\left(\varphi_{3}\right)$, with $\varphi_{2} \geq \varphi_{3}>0$

25. $B^{(1,1)}\left(\beta_{1}\right) \oplus B^{(1,1)}\left(\beta_{2}\right) \oplus B^{(0,2)}(\varphi \neq 0)$, with $\beta_{1} \geq \beta_{2}>0$

Again, let $\left\{\boldsymbol{e}_{1}, \ldots, \boldsymbol{e}_{6}\right\}$ be an ordered frame with $\boldsymbol{e}_{1}$ and $\boldsymbol{e}_{2}$ timelike and the rest spacelike. Then, using Table 4 , the above elements can be written as follows: 
1. $\varphi \boldsymbol{e}_{12},(\varphi>0)$;

2. $\beta \boldsymbol{e}_{13},(\beta>0)$;

3. $e_{12}-e_{23}$

4. $\varphi \boldsymbol{e}_{34},(\varphi>0)$

5. $e_{13}-e_{34}$

6. $-e_{12}-e_{13}+e_{24}+e_{34}$

7. $-e_{12}-e_{13}+e_{24}+e_{34}+\beta\left(e_{14}-e_{23}\right),(\beta>0)$;

8. $-\boldsymbol{e}_{12}-\boldsymbol{e}_{13}+\boldsymbol{e}_{24}+\boldsymbol{e}_{34}+\varphi\left(\boldsymbol{e}_{12}+\boldsymbol{e}_{34}\right),(\varphi \neq 0)$;

9. $\varphi\left(\boldsymbol{e}_{12}-\boldsymbol{e}_{34}\right)+\beta\left(\boldsymbol{e}_{14}-\boldsymbol{e}_{23}\right),(\beta>0, \varphi>0)$;

10. $\varphi_{1} \boldsymbol{e}_{12}+\varphi_{2} \boldsymbol{e}_{34},\left(\varphi_{i}>0\right)$;

11. $\beta_{1} \boldsymbol{e}_{13}+\beta_{2} \boldsymbol{e}_{24},\left(\beta_{1} \geq \beta_{2}>0\right)$;

12. $\beta \boldsymbol{e}_{13}+\varphi \boldsymbol{e}_{45},(\beta>0, \varphi>0)$;

13. $\boldsymbol{e}_{12}+\boldsymbol{e}_{13}+\boldsymbol{e}_{15}-\boldsymbol{e}_{24}-\boldsymbol{e}_{34}-\boldsymbol{e}_{45}$;

14. $\boldsymbol{e}_{12}-\boldsymbol{e}_{23}+\varphi \boldsymbol{e}_{45},(\varphi>0)$;

15. $\boldsymbol{e}_{13}-\boldsymbol{e}_{34}+\beta \boldsymbol{e}_{25},(\beta>0)$;

16. $\varphi_{1} \boldsymbol{e}_{34}+\varphi_{2} \boldsymbol{e}_{56},\left(\varphi_{1} \geq \varphi_{2}>0\right)$;

17. $\boldsymbol{e}_{13}-\boldsymbol{e}_{34}+\varphi \boldsymbol{e}_{56},(\varphi>0)$;

18. $\varphi\left(\mp e_{12}+e_{34}+e_{56}\right)+e_{15}-e_{35} \pm e_{26}-e_{46},(\varphi \neq 0)$

19. $-\boldsymbol{e}_{12}-\boldsymbol{e}_{13}+\boldsymbol{e}_{24}+\boldsymbol{e}_{34}+\beta\left(\boldsymbol{e}_{14}-\boldsymbol{e}_{23}\right)+\varphi \boldsymbol{e}_{56},(\beta>0, \varphi \neq 0)$;

20. $-\boldsymbol{e}_{12}-\boldsymbol{e}_{13}+\boldsymbol{e}_{24}+\boldsymbol{e}_{34}+\varphi_{1}\left(\boldsymbol{e}_{12}+\boldsymbol{e}_{34}\right)+\varphi_{2} \boldsymbol{e}_{56},\left(\varphi_{i} \neq 0\right)$;

21. $-e_{12}-e_{13}+e_{24}+e_{34}+\varphi e_{56},(\varphi \neq 0)$;

22. $\varphi_{1}\left(\boldsymbol{e}_{12}-\boldsymbol{e}_{34}\right)+\beta\left(\boldsymbol{e}_{14}-\boldsymbol{e}_{23}\right)+\varphi_{2} \boldsymbol{e}_{56},\left(\beta>0, \varphi_{1}>0, \varphi_{2} \neq 0\right)$;

23. $\boldsymbol{e}_{13}-\boldsymbol{e}_{34}+\boldsymbol{e}_{25}-\boldsymbol{e}_{56}$;

24. $\varphi_{1} \boldsymbol{e}_{12}+\varphi_{2} \boldsymbol{e}_{34}+\varphi_{3} \boldsymbol{e}_{56},\left(\varphi_{2} \geq \varphi_{3}>0, \varphi_{1} \neq 0\right)$;

25. $\beta_{1} \boldsymbol{e}_{13}+\beta_{2} \boldsymbol{e}_{24}+\varphi \boldsymbol{e}_{56},\left(\varphi \neq 0, \beta_{1} \geq \beta_{2}>0\right)$;

Clearly by letting some of the parameters become 0 , we can subsume some of these cases into others; but we prefer not to do this at this stage. The above list is in one-to-one correspondence with the conjugacy classes of nonzero elements of $\mathfrak{s o}(2,4)$ under $\mathrm{SO}(2,4)$. 


\subsubsection{Causal properties of orbits}

Similar considerations as those explained in the previous section on norms allow us to immediately write the norms of the first 15 Killing vector fields with the proviso that $R^{2}$ becomes $R^{2}+x_{6}^{2}$ with respect to the $\mathrm{AdS}_{4}$ norms. All cases but (18) involve no new computations, just adding results of previous computations. At the end of the day, one obtains the following norms:

1. $|\xi|^{2}=-\varphi^{2}\left(R^{2}+x_{3}^{2}+x_{4}^{2}+x_{5}^{2}+x_{6}^{2}\right)$, which is unbounded below;

2. $|\xi|^{2}=\beta^{2}\left(R^{2}-x_{2}^{2}+x_{4}^{2}+x_{5}^{2}+x_{6}^{2}\right)$, which is unbounded below;

3. $|\xi|^{2}=-\left(x_{3}+x_{1}\right)^{2}$, which is unbounded below;

4. $|\xi|^{2}=\varphi^{2}\left(x_{3}^{2}+x_{4}^{2}\right) \geq 0$;

5. $|\xi|^{2}=\left(x_{1}+x_{4}\right)^{2} \geq 0 ;$

6. $|\xi|^{2}=0$;

7. $|\xi|^{2}=\beta^{2}\left(R^{2}+x_{5}^{2}+x_{6}^{2}\right)+4 \beta\left(x_{1}+x_{4}\right)\left(x_{3}+x_{2}\right)$, which is unbounded below;

8. $|\xi|^{2}=-\varphi^{2}\left(R^{2}+x_{5}^{2}+x_{6}^{2}\right)+2 \varphi\left(\left(x_{1}+x_{4}\right)^{2}+\left(x_{3}+x_{2}\right)^{2}\right)$, which is unbounded below;

9. $|\xi|^{2}=\left(\beta^{2}-\varphi^{2}\right)\left(R^{2}+x_{5}^{2}+x_{6}^{2}\right)-4 \beta \varphi\left(x_{1} x_{3}+x_{2} x_{4}\right)$, which is unbounded below;

10. $|\xi|^{2}=-\varphi_{2}^{2}\left(R^{2}+x_{5}^{2}+x_{6}^{2}\right)+\left(\varphi_{1}^{2}-\varphi_{2}^{2}\right)\left(x_{3}^{2}+x_{4}^{2}\right)$, which is unbounded below;

11. $|\xi|^{2}=\beta_{1}^{2}\left(R^{2}+x_{5}^{2}+x_{6}^{2}\right)+\left(\beta_{2}^{2}-\beta_{1}^{2}\right)\left(x_{2}^{2}-x_{4}^{2}\right)$, which is $\geq \beta_{1}^{2} R^{2}$ if $\beta_{1}=\beta_{2}$ and unbounded below otherwise;

12. $|\xi|^{2}=\beta^{2}\left(x_{1}^{2}-x_{3}^{2}\right)+\varphi^{2}\left(x_{4}^{2}+x_{5}^{2}\right)$, which is unbounded below;

13. $|\xi|^{2}=\left(x_{1}-x_{4}\right)^{2}-4\left(x_{2}+x_{3}\right) x_{5}$, which is unbounded below;

14. $|\xi|^{2}=-\left(x_{1}+x_{3}\right)^{2}+\varphi^{2}\left(x_{4}^{2}+x_{5}^{2}\right)$, which is unbounded below;

15. $|\xi|^{2}=\left(x_{1}+x_{4}\right)^{2}+\beta^{2}\left(x_{2}^{2}-x_{5}^{2}\right)$, which is unbounded below;

16. $|\xi|^{2}=\varphi_{1}^{2}\left(x_{3}^{2}+x_{4}^{2}\right)+\varphi_{2}^{2}\left(x_{5}^{2}+x_{6}^{2}\right) \geq 0 ;$

17. $|\xi|^{2}=\left(x_{1}+x_{4}\right)^{2}+\varphi^{2}\left(x_{5}^{2}+x_{6}^{2}\right) \geq 0 ;$ 
18. $|\xi|^{2}=-\varphi^{2} R^{2}+\left(x_{1}-x_{3}\right)^{2}+\left(x_{4} \mp x_{2}\right)^{2}-4 \varphi\left(\left(x_{4} \mp x_{2}\right) x_{5}+\left(x_{1}-x_{3}\right) x_{6}\right)$, which is unbounded below, since in the subspace of $\mathrm{AdS}_{5}$ where $x_{4} \mp$ $x_{2}=0$ and $x_{1}-x_{3}=-2 \varphi x_{6}$ we can take $\left|x_{6}\right|$ as large as desired;

19. $|\xi|^{2}=\beta^{2} R^{2}+4 \beta\left(x_{1}+x_{4}\right)\left(x_{2}+x_{3}\right)+\left(\varphi^{2}+\beta^{2}\right)\left(x_{5}^{2}+x_{6}^{2}\right)$, which is unbounded below;

20. $\left.|\xi|^{2}=-\varphi_{1}^{2} R^{2}+2 \varphi_{1}\left(\left(x_{1}+x_{4}\right)^{2}+\left(x_{2}+x_{3}\right)^{2}\right)\right)+\left(\varphi_{2}^{2}-\varphi_{1}^{2}\right)\left(x_{5}^{2}+x_{6}^{2}\right)$, which is $\geq-\varphi_{1}^{2} R^{2}$ provided that $\left|\varphi_{2}\right| \geq \varphi_{1}>0$ and unbounded below otherwise;

21. $|\xi|^{2}=\varphi^{2}\left(x_{5}^{2}+x_{6}^{2}\right) \geq 0 ;$

22. $|\xi|^{2}=\left(\beta^{2}-\varphi_{1}^{2}\right) R^{2}+\left(\beta^{2}-\varphi_{1}^{2}+\varphi_{2}^{2}\right)\left(x_{5}^{2}+x_{6}^{2}\right)-4 \beta \varphi_{1}\left(x_{1} x_{3}+x_{2} x_{4}\right)$, which is unbounded below;

23. $|\xi|^{2}=\left(x_{1}+x_{4}\right)^{2}+\left(x_{2}+x_{6}\right)^{2}>0$;

24. $|\xi|^{2}=-\varphi_{1}^{2} R^{2}+\left(\varphi_{2}^{2}-\varphi_{1}^{2}\right)\left(x_{3}^{2}+x_{4}^{2}\right)+\left(\varphi_{3}^{2}-\varphi_{1}^{2}\right)\left(x_{5}^{2}+x_{6}^{2}\right)$, which is $\geq-\varphi_{1}^{2} R^{2}$ provided that $\varphi_{3} \geq \varphi_{2} \geq\left|\varphi_{1}\right|>0$; and

25. $|\xi|^{2}=\beta_{1}^{2} R^{2}+\left(\beta_{2}^{2}-\beta_{1}^{2}\right)\left(x_{2}^{2}-x_{4}^{2}\right)+\left(\varphi^{2}+\beta_{1}^{2}\right)\left(x_{5}^{2}+x_{6}^{2}\right)$, which is $\geq \beta_{1}^{2} R^{2}$ if $\beta_{2}=\beta_{1}$ and unbounded below otherwise.

Since we will be applying these results to the reductions of $\mathrm{AdS}_{5} \times S^{5}$ and $S^{5}$ does possess Killing vectors without zeroes, we will be needing those Killing vectors on $\mathrm{AdS}_{5}$ whose norms are bounded below. From the above list we see immediately that these are (4), (5), (6), (11) for $\beta_{1}=\beta_{2},(16)$, (17), (20) for $\left|\varphi_{2}\right| \geq \varphi_{1}>0$, (21), (23), (24) for $\varphi_{2} \geq \varphi_{3} \geq\left|\varphi_{1}\right|>0$, and (25) for $\beta_{1}=\beta_{2}$.

\subsection{One-parameter subgroups of $\mathrm{SO}(2,6)$}

\subsubsection{Adjoint orbits of $\mathfrak{s o}(2,6)$}

The following decompositions are possible for a skew-symmetric endomorphism $B$ up to conjugation by $\mathrm{SO}(2,6)$ :

- $[(2,4) \oplus(0,2)]_{\mathrm{SO}}=(2,4)_{\mathrm{O}} \oplus(0,2)$

- $(2,4)_{\mathrm{O}} \oplus 2(0,1)$

- $(2,3) \oplus(0,2)_{\mathrm{O}} \oplus(0,1)$ 
- $(2,3) \oplus 3(0,1)$

- $[(2,2) \oplus 2(0,2)]_{\mathrm{SO}}=(2,2)_{\mathrm{O}} \oplus[2(0,2)]_{\mathrm{SO}}$

- $(2,2)_{\mathrm{O}} \oplus(0,2) \oplus 2(0,1)$

- $(2,2)_{\mathrm{O}} \oplus 4(0,1)$

- $(2,1) \oplus[2(0,2)]_{\mathrm{O}} \oplus(0,1)$

- $(2,1) \oplus(0,2)_{\mathrm{O}} \oplus 3(0,1)$

- $(2,1) \oplus 5(0,1)$

- $2(1,2) \oplus(0,2)_{\mathrm{O}}$

- $2(1,2) \oplus 2(0,1)$

- $(1,2) \oplus[2(0,2)]_{\mathrm{O}} \oplus(1,0)$

- $(1,2) \oplus(1,1)_{\mathrm{O}} \oplus(0,2)_{\mathrm{O}} \oplus(0,1)$

- $(1,2) \oplus(1,1)_{\mathrm{O}} \oplus 3(0,1)$

- $(1,2) \oplus(0,2)_{\mathrm{O}} \oplus(1,0) \oplus 2(0,1)$

- $(1,2) \oplus(1,0) \oplus 4(0,1)$

- $(2,0) \oplus[3(0,2)]_{\mathrm{O}}$

- $(2,0)_{\mathrm{O}} \oplus[2(0,2)]_{\mathrm{O}} \oplus 2(0,1)$

- $(2,0)_{\mathrm{O}} \oplus(0,2)_{\mathrm{O}} \oplus 4(0,1)$

- $(2,0)_{\mathrm{O}} \oplus 6(0,1)$

- $[2(1,1) \oplus 2(0,2)]_{\mathrm{SO}}$

- $[2(1,1)]_{\mathrm{O}} \oplus 4(0,1)$

- $[2(1,1) \oplus(0,2)]_{\mathrm{O}} \oplus 2(0,1)$

- $[(1,1) \oplus 2(0,2)]_{\mathrm{O}} \oplus(1,0) \oplus(0,1)$

- $[(1,1) \oplus(0,2)]_{\mathrm{O}} \oplus(1,0) \oplus 3(0,1)$

- $(1,1)_{\mathrm{O}} \oplus(1,0) \oplus 5(0,1)$

- $[3(0,2)]_{\mathrm{O}} \oplus 2(1,0)$

- $[2(0,2)]_{\mathrm{O}} \oplus 2(1,0) \oplus 2(0,1)$ 
- $(0,2)_{\mathrm{O}} \oplus 2(1,0) \oplus 4(0,1)$

We still have to work out a few of the blocks which are not elementary, namely $[2(0,2)]_{\mathrm{SO}},[3(0,2)]_{\mathrm{O}},[2(1,1) \oplus 2(0,2)]_{\mathrm{SO}},[2(1,1) \oplus(0,2)]_{\mathrm{O}},[(1,1) \oplus$ $2(0,2)]_{\mathrm{O}}$ and $[(1,1) \oplus(0,2)]_{\mathrm{O}}$. We will simply state the results, which are easily verified as was done in previous cases already treated in detail:

- $[2(0,2)]_{\mathrm{SO}}$ is represented by $B^{(0,2)}\left(\varphi_{1}\right) \oplus B^{(0,2)}\left(\varphi_{2}\right)$, where $\varphi_{1} \geq\left|\varphi_{2}\right|>$ 0 ;

- $[3(0,2)]_{\mathrm{O}}$ is represented by $B^{(0,2)}\left(\varphi_{1}\right) \oplus B^{(0,2)}\left(\varphi_{2}\right) \oplus B^{(0,2)}\left(\varphi_{3}\right)$, where $\varphi_{1} \geq \varphi_{2} \geq \varphi_{3}>0$

- $[2(1,1) \oplus 2(0,2)]_{\text {SO }}$ is represented by $B^{(1,1)}\left(\beta_{1}\right) \oplus B^{(1,1)}\left(\beta_{2}\right) \oplus B^{(0,2)}\left(\varphi_{1}\right) \oplus$ $B^{(0,2)}\left(\varphi_{2}\right)$, where $\beta_{1} \geq \beta_{2}>0$ and $\varphi_{1} \geq\left|\varphi_{2}\right|>0$;

- $[2(1,1) \oplus(0,2)]_{\mathrm{O}}$ is represented by $B^{(1,1)}\left(\beta_{1}\right) \oplus B^{(1,1)}\left(\beta_{2}\right) \oplus B^{(0,2)}(\varphi)$, where $\beta_{1} \geq \beta_{2}>0$ and $\varphi>0$;

- $[(1,1) \oplus 2(0,2)]_{\mathrm{O}}$ is represented by $B^{(1,1)}(\beta) \oplus B^{(0,2)}\left(\varphi_{1}\right) \oplus B^{(0,2)}\left(\varphi_{2}\right)$, where $\beta>0$ and $\varphi_{1} \geq \varphi_{2}>0$; and

- $[(1,1) \oplus(0,2)]_{\mathrm{O}}$ is represented by $B^{(1,1)}(\beta) \oplus B^{(0,2)}(\varphi)$, where $\beta>0$ and $\varphi>0$.

We are now able to finally list all the possible elements of $\mathfrak{s o}(2,6)$ up to the action of $\mathrm{SO}(2,6)$ :

1. $B^{(2,0)}\left(\varphi_{1}>0\right) \oplus 6 B^{(0,1)}$

2. $B^{(1,1)}(\beta>0) \oplus 5 B^{(0,1)} \oplus B^{(1,0)}$

3. $B^{(2,1)} \oplus 5 B^{(0,1)}$

4. $B^{(0,2)}(\varphi>0) \oplus 2 B^{(1,0)} \oplus 4 B^{(0,1)}$

5. $B^{(1,2)} \oplus B^{(1,0)} \oplus 4 B^{(0,1)}$

6. $B_{+}^{(2,2)} \oplus 4 B^{(0,1)}$

7. $B_{+}^{(2,2)}(\beta>0) \oplus 4 B^{(0,1)}$

8. $B_{+}^{(2,2)}(\varphi \neq 0) \oplus 4 B^{(0,1)}$

9. $B_{+}^{(2,2)}(\beta>0, \varphi>0) \oplus 4 B^{(0,1)}$ 
10. $B^{(2,0)}\left(\varphi_{1}>0\right) \oplus B^{(0,2)}\left(\varphi_{2}>0\right) \oplus 4 B^{(0,1)}$

11. $B^{(1,1)}\left(\beta_{1}\right) \oplus B^{(1,1)}\left(\beta_{2}\right) \oplus 4 B^{(0,1)}$ with $\beta_{1} \geq \beta_{2}>0$

12. $B^{(1,1)}(\beta>0) \oplus B^{(0,2)}(\varphi>0) \oplus 3 B^{(0,1)} \oplus B^{(1,0)}$

13. $B^{(2,3)} \oplus 3 B^{0,1}$

14. $B^{(2,1)} \oplus B^{(0,2)}(\varphi>0) \oplus 3 B^{(0,1)}$

15. $B^{(1,2)} \oplus B^{(1,1)}(\beta>0) \oplus 3 B^{(0,1)}$

16. $B^{(0,2)}\left(\varphi_{1}\right) \oplus B^{(0,2)}\left(\varphi_{2}\right) \oplus 2 B^{(0,1)} \oplus 2 B^{(1,0)}$, where $\varphi_{1} \geq \varphi_{2}>0$

17. $B^{(1,2)} \oplus B^{(0,2)}(\varphi>0) \oplus B^{(1,0)} \oplus 2 B^{(0,1)}$

18. $B_{+}^{(2,4)}(\varphi \neq 0) \oplus 2 B^{(0,1)}$

19. $B_{+}^{(2,2)}(\beta>0) \oplus B^{(0,2)}(\varphi \neq 0) \oplus 2 B^{(0,1)}$

20. $B_{+}^{(2,2)}\left(\varphi_{1} \neq 0\right) \oplus B^{(0,2)}\left(\varphi_{2} \neq 0\right) \oplus 2 B^{(0,1)}$

21. $B_{+}^{(2,2)} \oplus B^{(0,2)}(\varphi \neq 0) \oplus 2 B^{(0,1)}$

22. $B_{+}^{(2,2)}\left(\beta>0, \varphi_{1}>0\right) \oplus B^{(0,2)}\left(\varphi_{2} \neq 0\right) \oplus 2 B^{(0,1)}$

23. $2 B^{(1,2)} \oplus 2 B^{(0,1)}$

24. $B^{(2,0)}\left(\varphi_{1}>0\right) \oplus B^{(0,2)}\left(\varphi_{2}\right) \oplus B^{(0,2)}\left(\varphi_{3}\right) \oplus 2 B^{(0,1)}$ with $\varphi_{2} \geq \varphi_{3}>0$

25. $B^{(1,1)}\left(\beta_{1}\right) \oplus B^{(1,1)}\left(\beta_{2}\right) \oplus B^{(0,2)}(\varphi>0) \oplus 2 B^{(0,1)}$, with $\beta_{1} \geq \beta_{2}>0$

26. $B^{(1,1)}(\beta>0) \oplus B^{(0,2)}\left(\varphi_{1}\right) \oplus B^{(0,2)}\left(\varphi_{2}\right) \oplus B^{(0,1)} \oplus B^{(1,0)}$, with $\varphi_{1} \geq \varphi_{2}>0$

27. $B^{(2,3)} \oplus B^{(0,2)}(\varphi>0) \oplus B^{0,1}$

28. $B^{(2,1)} \oplus B^{(0,2)}\left(\varphi_{1}\right) \oplus B^{(0,2)}\left(\varphi_{2}\right) \oplus B^{(0,1)}$ with $\varphi_{1} \geq \varphi_{2}>0$

29. $B^{(1,2)} \oplus B^{(1,1)}(\beta>0) \oplus B^{(0,2)}(\varphi>0) \oplus B^{(0,1)}$

30. $B^{(0,2)}\left(\varphi_{1}\right) \oplus B^{(0,2)}\left(\varphi_{2}\right) \oplus B^{(0,2)}\left(\varphi_{3}\right) \oplus 2 B^{(1,0)}$, where $\varphi_{1} \geq \varphi_{2} \geq \varphi_{3}>0$

31. $B^{(1,2)} \oplus B^{(0,2)}\left(\varphi_{1}\right) \oplus B^{(0,2)}\left(\varphi_{2}\right) \oplus B^{(1,0)}$, with $\varphi_{1} \geq \varphi_{2}>0$

32. $B_{+}^{(2,4)}\left(\varphi_{1} \neq 0\right) \oplus B^{(0,2)}\left(\varphi_{2} \neq 0\right)$

33. $B_{+}^{(2,2)} \oplus B^{(0,2)}\left(\varphi_{1}\right) \oplus B^{(0,2)}\left(\varphi_{2}\right)$ with $\varphi_{1} \geq\left|\varphi_{2}\right|>0$

34. $B_{+}^{(2,2)}(\beta>0) \oplus B^{(0,2)}\left(\varphi_{1}\right) \oplus B^{(0,2)}\left(\varphi_{2}\right)$ with $\varphi_{1} \geq\left|\varphi_{2}\right|>0$ 
35. $B_{+}^{(2,2)}\left(\varphi_{1} \neq 0\right) \oplus B^{(0,2)}\left(\varphi_{2}\right) \oplus B^{(0,2)}\left(\varphi_{3}\right)$ with $\varphi_{2} \geq\left|\varphi_{3}\right|>0$

36. $B_{+}^{(2,2)}\left(\beta>0, \varphi_{1}>0\right) \oplus B^{(0,2)}\left(\varphi_{2}\right) \oplus B^{(0,2)}\left(\varphi_{3}\right)$ with $\varphi_{2} \geq\left|\varphi_{3}\right|>0$

37. $2 B^{(1,2)} \oplus B^{(0,2)}(\varphi>0)$

38. $B^{(2,0)}\left(\varphi_{1} \neq 0\right) \oplus B^{(0,2)}\left(\varphi_{2}\right) \oplus B^{(0,2)}\left(\varphi_{3}\right) \oplus B^{(0,2)}\left(\varphi_{4}\right)$ with $\varphi_{2} \geq \varphi_{3} \geq$ $\varphi_{4}>0$

39. $B^{(1,1)}\left(\beta_{1}\right) \oplus B^{(1,1)}\left(\beta_{2}\right) \oplus B^{(0,2)}\left(\varphi_{1}\right) \oplus B^{(0,2)}\left(\varphi_{2}\right)$, with $\beta_{1} \geq \beta_{2}>0$ and $\varphi_{1} \geq\left|\varphi_{2}\right|>0$

where we have again reordered them in such a way that the embeddings $\mathfrak{s o}(2,2) \subset \mathfrak{s o}(2,3) \subset \mathfrak{s o}(2,4) \subset \mathfrak{s o}(2,6)$ are compatible with the labelling; in particular, cases (1)-(25) correspond to the embedding in $\mathfrak{s o}(2,6)$ of the corresponding elements in $\mathfrak{s o}(2,4)$.

Again we rewrite this list in a more traditional notation. Let $\left\{\boldsymbol{e}_{1}, \ldots, \boldsymbol{e}_{8}\right\}$ be an ordered frame with $\boldsymbol{e}_{1}$ and $\boldsymbol{e}_{2}$ timelike and the rest spacelike. Then, using Table 4, the above elements can be written as follows:
1. $\varphi \boldsymbol{e}_{12},(\varphi>0)$;
2. $\beta \boldsymbol{e}_{13},(\beta>0)$;
3. $e_{12}-e_{23}$
4. $\varphi \boldsymbol{e}_{34},(\varphi>0)$;
5. $e_{13}-e_{34}$
6. $-e_{12}-e_{13}+e_{24}+e_{34}$
7. $-e_{12}-e_{13}+e_{24}+e_{34}+\beta\left(e_{14}-e_{23}\right),(\beta>0)$;
8. $-\boldsymbol{e}_{12}-\boldsymbol{e}_{13}+\boldsymbol{e}_{24}+\boldsymbol{e}_{34}+\varphi\left(\boldsymbol{e}_{12}+\boldsymbol{e}_{34}\right)(\varphi \neq 0)$;
9. $\varphi\left(\boldsymbol{e}_{12}-\boldsymbol{e}_{34}\right)+\beta\left(\boldsymbol{e}_{14}-\boldsymbol{e}_{23}\right),(\beta>0, \varphi>0)$;
10. $\varphi_{1} \boldsymbol{e}_{12}+\varphi_{2} \boldsymbol{e}_{34},\left(\varphi_{i}>0\right)$;
11. $\beta_{1} \boldsymbol{e}_{13}+\beta_{2} \boldsymbol{e}_{24},\left(\beta_{1} \geq \beta_{2}>0\right)$;
12. $\beta \boldsymbol{e}_{13}+\varphi \boldsymbol{e}_{45},(\varphi>0, \beta>0)$;
13. $e_{12}+e_{13}+e_{15}-e_{24}-e_{34}-e_{45}$
14. $\boldsymbol{e}_{12}-\boldsymbol{e}_{23}+\varphi \boldsymbol{e}_{45},(\varphi>0)$; 
15. $\boldsymbol{e}_{13}-\boldsymbol{e}_{34}+\beta \boldsymbol{e}_{25},(\beta>0)$

16. $\varphi_{1} \boldsymbol{e}_{34}+\varphi_{2} \boldsymbol{e}_{56},\left(\varphi_{1} \geq \varphi_{2}>0\right)$;

17. $\boldsymbol{e}_{13}-\boldsymbol{e}_{34}+\varphi \boldsymbol{e}_{56},(\varphi>0)$;

18. $\varphi\left(-\boldsymbol{e}_{12}+\boldsymbol{e}_{34}+\boldsymbol{e}_{56}\right)+\boldsymbol{e}_{15}-\boldsymbol{e}_{35}+\boldsymbol{e}_{26}-\boldsymbol{e}_{46},(\varphi \neq 0)$;

19. $-\boldsymbol{e}_{12}-\boldsymbol{e}_{13}+\boldsymbol{e}_{24}+\boldsymbol{e}_{34}+\beta\left(\boldsymbol{e}_{14}-\boldsymbol{e}_{23}\right)+\varphi \boldsymbol{e}_{56},(\beta>0, \varphi \neq 0)$;

20. $-\boldsymbol{e}_{12}-\boldsymbol{e}_{13}+\boldsymbol{e}_{24}+\boldsymbol{e}_{34}+\varphi_{1}\left(\boldsymbol{e}_{12}+\boldsymbol{e}_{34}\right)+\varphi_{2} \boldsymbol{e}_{56},\left(\varphi_{i} \neq 0\right)$;

21. $-e_{12}-e_{13}+e_{24}+e_{34}+\varphi e_{56},(\varphi \neq 0)$;

22. $\varphi_{1}\left(\boldsymbol{e}_{12}-\boldsymbol{e}_{34}\right)+\beta\left(\boldsymbol{e}_{14}-\boldsymbol{e}_{23}\right)+\varphi_{2} \boldsymbol{e}_{56},\left(\beta>0, \varphi_{1}>0, \varphi_{2} \neq 0\right)$;

23. $e_{13}-e_{34}+e_{25}-e_{56}$;

24. $\varphi_{1} \boldsymbol{e}_{12}+\varphi_{2} \boldsymbol{e}_{34}+\varphi_{3} \boldsymbol{e}_{56},\left(\varphi_{1}>0, \varphi_{2} \geq \varphi_{3}>0\right)$;

25. $\beta_{1} \boldsymbol{e}_{13}+\beta_{2} \boldsymbol{e}_{24}+\varphi \boldsymbol{e}_{56},\left(\varphi>0, \beta_{1} \geq \beta_{2}>0\right)$;

26. $\beta \boldsymbol{e}_{13}+\varphi_{1} \boldsymbol{e}_{56}+\varphi_{2} \boldsymbol{e}_{78},\left(\varphi_{1} \geq \varphi_{2}>0, \beta>0\right)$;

27. $\boldsymbol{e}_{12}+\boldsymbol{e}_{13}+\boldsymbol{e}_{15}-\boldsymbol{e}_{24}-\boldsymbol{e}_{34}-\boldsymbol{e}_{45}+\varphi \boldsymbol{e}_{78},(\varphi>0)$;

28. $\boldsymbol{e}_{12}-\boldsymbol{e}_{23}+\varphi_{1} \boldsymbol{e}_{45}+\varphi_{2} \boldsymbol{e}_{67},\left(\varphi_{1} \geq \varphi_{2}>0\right)$;

29. $\boldsymbol{e}_{13}-\boldsymbol{e}_{34}+\beta \boldsymbol{e}_{25}+\varphi \boldsymbol{e}_{67},(\beta>0, \varphi>0)$;

30. $\varphi_{1} \boldsymbol{e}_{34}+\varphi_{2} \boldsymbol{e}_{56}+\varphi_{3} \boldsymbol{e}_{78},\left(\varphi_{1} \geq \varphi_{2} \geq \varphi_{3}>0\right)$;

31. $\boldsymbol{e}_{13}-\boldsymbol{e}_{34}+\varphi_{1} \boldsymbol{e}_{56}+\varphi_{2} \boldsymbol{e}_{78},\left(\varphi_{1} \geq \varphi_{2}>0\right)$;

32. $\varphi_{1}\left(-\boldsymbol{e}_{12}+\boldsymbol{e}_{34}+\boldsymbol{e}_{56}\right)+\boldsymbol{e}_{15}-\boldsymbol{e}_{35}+\boldsymbol{e}_{26}-\boldsymbol{e}_{46}+\varphi_{2} \boldsymbol{e}_{78},\left(\varphi_{i} \neq 0\right)$;

33. $-\boldsymbol{e}_{12}-\boldsymbol{e}_{13}+\boldsymbol{e}_{24}+\boldsymbol{e}_{34}+\varphi_{1} \boldsymbol{e}_{56}+\varphi_{2} \boldsymbol{e}_{78},\left(\varphi_{1} \geq\left|\varphi_{2}\right|>0\right)$;

34. $-\boldsymbol{e}_{12}-\boldsymbol{e}_{13}+\boldsymbol{e}_{24}+\boldsymbol{e}_{34}+\beta\left(\boldsymbol{e}_{14}-\boldsymbol{e}_{23}\right)+\varphi_{1} \boldsymbol{e}_{56}+\varphi_{2} \boldsymbol{e}_{78},\left(\beta>0, \varphi_{1} \geq\right.$ $\left.\left|\varphi_{2}\right|>0\right)$;

35. $-\boldsymbol{e}_{12}-\boldsymbol{e}_{13}+\boldsymbol{e}_{24}+\boldsymbol{e}_{34}+\varphi_{1}\left(\boldsymbol{e}_{12}+\boldsymbol{e}_{34}\right)+\varphi_{2} \boldsymbol{e}_{56}+\varphi_{3} \boldsymbol{e}_{78},\left(\varphi_{1} \neq 0\right.$, $\left.\varphi_{2} \geq\left|\varphi_{3}\right|>0\right)$

36. $\varphi_{1}\left(\boldsymbol{e}_{12}-\boldsymbol{e}_{34}\right)+\beta\left(\boldsymbol{e}_{14}-\boldsymbol{e}_{23}\right)+\varphi_{2} \boldsymbol{e}_{56}+\varphi_{3} \boldsymbol{e}_{78},\left(\beta>0, \varphi_{1}>0, \varphi_{2} \geq\right.$ $\left.\left|\varphi_{3}\right|>0\right)$

37. $\boldsymbol{e}_{13}-\boldsymbol{e}_{34}+\boldsymbol{e}_{25}-\boldsymbol{e}_{56}+\varphi \boldsymbol{e}_{78},(\varphi>0)$

38. $\varphi_{1} \boldsymbol{e}_{12}+\varphi_{2} \boldsymbol{e}_{34}+\varphi_{3} \boldsymbol{e}_{56}+\varphi_{4} \boldsymbol{e}_{78},\left(\varphi_{1} \neq 0, \varphi_{2} \geq \varphi_{3} \geq \varphi_{4}>0\right)$; 
39. $\beta_{1} \boldsymbol{e}_{13}+\beta_{2} \boldsymbol{e}_{24}+\varphi_{1} \boldsymbol{e}_{56}+\varphi_{2} \boldsymbol{e}_{78},\left(\varphi_{1} \geq\left|\varphi_{2}\right|>0, \beta_{1} \geq \beta_{2}>0\right)$;

Again it is clear that by letting some of the parameters become 0, we can subsume some of these cases into others; but we prefer not to do this at this stage. The above list is in one-to-one correspondence with the conjugacy classes of nonzero elements of $\mathfrak{s o}(2,6)$ under $\mathrm{SO}(2,6)$.

\subsubsection{Causal properties of orbits}

Similar considerations as those explained in the previous section on norms allow us to immediately write the norms of the first 25 Killing vector fields with the proviso that $R^{2}$ becomes $R^{2}+x_{7}^{2}+x_{8}^{2}$ with respect to the $\mathrm{AdS}_{5}$ norms. All other cases involve no new computations, just adding results of previous computations. At the end of the day (or night!), one obtains the following norms:

1. $|\xi|^{2}=-\varphi^{2}\left(R^{2}+x_{3}^{2}+x_{4}^{2}+x_{5}^{2}+x_{6}^{2}+x_{7}^{2}+x_{8}^{2}\right)$, which is unbounded below;

2. $|\xi|^{2}=\beta^{2}\left(R^{2}-x_{2}^{2}+x_{4}^{2}+x_{5}^{2}+x_{6}^{2}+x_{7}^{2}+x_{8}^{2}\right)$, which is unbounded below;

3. $|\xi|^{2}=-\left(x_{3}+x_{1}\right)^{2}$, which is unbounded below;

4. $|\xi|^{2}=\varphi^{2}\left(x_{3}^{2}+x_{4}^{2}\right) \geq 0$;

5. $|\xi|^{2}=\left(x_{1}+x_{4}\right)^{2} \geq 0$

6. $|\xi|^{2}=0$;

7. $|\xi|^{2}=\beta^{2}\left(R^{2}+x_{5}^{2}+x_{6}^{2}+x_{7}^{2}+x_{8}^{2}\right)+4 \beta\left(x_{1}+x_{4}\right)\left(x_{3}+x_{2}\right)$, which is unbounded below;

8. $|\xi|^{2}=-\varphi^{2}\left(R^{2}+x_{5}^{2}+x_{6}^{2}+x_{7}^{2}+x_{8}^{2}\right)+2 \varphi\left(\left(x_{1}+x_{4}\right)^{2}+\left(x_{3}+x_{2}\right)^{2}\right)$, which is unbounded below;

9. $|\xi|^{2}=\left(\beta^{2}-\varphi^{2}\right)\left(R^{2}+x_{5}^{2}+x_{6}^{2}+x_{7}^{2}+x_{8}^{2}\right)-4 \beta \varphi\left(x_{1} x_{3}+x_{2} x_{4}\right)$, which is unbounded below;

10. $|\xi|^{2}=-\varphi_{2}^{2}\left(R^{2}+x_{5}^{2}+x_{6}^{2}+x_{7}^{2}+x_{8}^{2}\right)+\left(\varphi_{1}^{2}-\varphi_{2}^{2}\right)\left(x_{3}^{2}+x_{4}^{2}\right)$, which is unbounded below;

11. $|\xi|^{2}=\beta_{1}^{2}\left(R^{2}+x_{5}^{2}+x_{6}^{2}+x_{7}^{2}+x_{8}^{2}\right)+\left(\beta_{2}^{2}-\beta_{1}^{2}\right)\left(x_{2}^{2}-x_{4}^{2}\right)$, which is $\geq \beta_{1}^{2} R^{2}$ if $\beta_{1}=\beta_{2}$ and unbounded below otherwise;

12. $|\xi|^{2}=\beta^{2}\left(x_{1}^{2}-x_{3}^{2}\right)+\varphi^{2}\left(x_{4}^{2}+x_{5}^{2}\right)$, which is unbounded below; 
13. $|\xi|^{2}=\left(x_{4}-x_{1}\right)^{2}-4\left(x_{2}+x_{3}\right) x_{5}$, which is unbounded below;

14. $|\xi|^{2}=-\left(x_{1}+x_{3}\right)^{2}+\varphi^{2}\left(x_{4}^{2}+x_{5}^{2}\right)$, which is unbounded below;

15. $|\xi|^{2}=\left(x_{1}+x_{4}\right)^{2}+\beta^{2}\left(x_{2}^{2}-x_{5}^{2}\right)$, which is unbounded below;

16. $|\xi|^{2}=\varphi_{1}^{2}\left(x_{3}^{2}+x_{4}^{2}\right)+\varphi_{2}^{2}\left(x_{5}^{2}+x_{6}^{2}\right) \geq 0 ;$

17. $|\xi|^{2}=\left(x_{1}+x_{4}\right)^{2}+\varphi^{2}\left(x_{5}^{2}+x_{6}^{2}\right) \geq 0$;

18. $|\xi|^{2}=-\varphi^{2}\left(R^{2}+x_{7}^{2}+x_{8}^{2}\right)+\left(x_{1}-x_{3}\right)^{2}+\left(x_{4}-x_{2}\right)^{2}-4 \varphi\left(\left(x_{4}-x_{2}\right) x_{5}+\right.$ $\left.\left(x_{1}-x_{3}\right) x_{6}\right)$, which is unbounded below;

19. $|\xi|^{2}=\beta^{2}\left(R^{2}+x_{7}^{2}+x_{8}^{2}\right)+4 \beta\left(x_{1}+x_{4}\right)\left(x_{2}+x_{3}\right)+\left(\varphi^{2}+\beta^{2}\right)\left(x_{5}^{2}+x_{6}^{2}\right)$, which is unbounded below;

20. $\left.|\xi|^{2}=-\varphi_{1}^{2}\left(R^{2}+x_{7}^{2}+x_{8}^{2}\right)+2 \varphi_{1}\left(\left(x_{1}+x_{4}\right)^{2}+\left(x_{2}+x_{3}\right)^{2}\right)\right)+\left(\varphi_{2}^{2}-\varphi_{1}^{2}\right)\left(x_{5}^{2}+\right.$ $\left.x_{6}^{2}\right)$, which is unbounded below;

21. $|\xi|^{2}=\varphi^{2}\left(x_{5}^{2}+x_{6}^{2}\right) \geq 0$;

22. $|\xi|^{2}=\left(\beta^{2}-\varphi_{1}^{2}\right)\left(R^{2}+x_{7}^{2}+x_{8}^{2}\right)+\left(\beta^{2}-\varphi_{1}^{2}+\varphi_{2}^{2}\right)\left(x_{5}^{2}+x_{6}^{2}\right)-4 \beta \varphi_{1}\left(x_{1} x_{3}+\right.$ $\left.x_{2} x_{4}\right)$, which is unbounded below;

23. $|\xi|^{2}=\left(x_{1}+x_{4}\right)^{2}+\left(x_{2}+x_{6}\right)^{2}>0$;

24. $|\xi|^{2}=-\varphi_{1}^{2}\left(R^{2}+x_{7}^{2}+x_{8}^{2}\right)+\left(\varphi_{2}^{2}-\varphi_{1}^{2}\right)\left(x_{3}^{2}+x_{4}^{2}\right)+\left(\varphi_{3}^{2}-\varphi_{1}^{2}\right)\left(x_{5}^{2}+x_{6}^{2}\right)$, which is $\geq-\varphi_{1}^{2} R^{2}$ provided that $\varphi_{2} \geq \varphi_{3} \geq\left|\varphi_{1}\right|>0$;

25. $|\xi|^{2}=\beta_{1}^{2}\left(R^{2}+x_{7}^{2}+x_{8}^{2}\right)+\left(\beta_{2}^{2}-\beta_{1}^{2}\right)\left(x_{2}^{2}-x_{4}^{2}\right)+\left(\varphi^{2}+\beta_{1}^{2}\right)\left(x_{5}^{2}+x_{6}^{2}\right)$, which is $\geq \beta_{1}^{2} R^{2}$ if $\beta_{2}=\beta_{1}$ and unbounded below otherwise;

26. $|\xi|^{2}=\beta^{2}\left(R^{2}-x_{2}^{2}+x_{4}^{2}\right)+\left(\varphi_{1}^{2}+\beta^{2}\right)\left(x_{5}^{2}+x_{6}^{2}\right)+\left(\varphi_{2}^{2}+\beta^{2}\right)\left(x_{7}^{2}+x_{8}^{2}\right)$, which is unbounded below;

27. $|\xi|^{2}=\left(x_{4}-x_{1}\right)^{2}-4 x_{5}\left(x_{2}+x_{3}\right)+\varphi^{2}\left(x_{7}^{2}+x_{8}^{2}\right)$, which is unbounded below;

28. $|\xi|^{2}=-\left(x_{1}+x_{3}\right)^{2}+\varphi_{1}\left(x_{4}^{2}+x_{5}^{2}\right)+\varphi_{2}\left(x_{6}^{2}+x_{7}^{2}\right)$, which is unbounded below;

29. $|\xi|^{2}=\left(x_{1}+x_{4}\right)^{2}+\beta^{2}\left(x_{2}^{2}-x_{5}^{2}\right)+\varphi^{2}\left(x_{6}^{2}+x_{7}^{2}\right)$, which is unbounded below;

30. $|\xi|^{2}=\varphi_{1}^{2}\left(x_{3}^{2}+x_{4}^{2}\right)+\varphi_{2}^{2}\left(x_{5}^{2}+x_{6}^{2}\right)+\varphi_{3}^{2}\left(x_{7}^{2}+x_{8}^{2}\right) \geq 0 ;$

31. $|\xi|^{2}=\left(x_{1}+x_{4}\right)^{2}+\varphi_{1}^{2}\left(x_{5}^{2}+x_{6}^{2}\right)+\varphi_{2}^{2}\left(x_{7}^{2}+x_{8}^{2}\right) \geq 0$;

32. $|\xi|^{2}=-\varphi_{1}^{2}\left(R^{2}+x_{7}^{2}+x_{8}^{2}\right)+\left(x_{1}-x_{3}\right)^{2}+\left(x_{4}-x_{2}\right)^{2}-4 \varphi_{1}\left(\left(x_{4}-x_{2}\right) x_{5}+\right.$ $\left.\left(x_{1}-x_{3}\right) x_{6}\right)+\varphi_{2}^{2}\left(x_{7}^{8}+x_{8}^{2}\right)$, which is unbounded below; 
33. $|\xi|^{2}=\varphi_{1}^{2}\left(x_{5}^{2}+x_{6}^{2}\right)+\varphi_{2}^{2}\left(x_{7}^{2}+x_{8}^{2}\right) \geq 0 ;$

34. $|\xi|^{2}=\beta^{2} R^{2}+4 \beta\left(x_{1}+x_{4}\right)\left(x_{2}+x_{3}\right)+\left(\varphi_{1}^{2}+\beta^{2}\right)\left(x_{5}^{2}+x_{6}^{2}\right)+\left(\varphi_{2}^{2}+\beta^{2}\right)\left(x_{7}^{2}+x_{8}^{2}\right)$, which is unbounded below;

35. $|\xi|^{2}=-\varphi_{1}^{2} R^{2}+2 \varphi_{1}\left(\left(x_{1}+x_{4}\right)^{2}+\left(x_{2}+x_{3}\right)^{2}\right)+\left(\varphi_{2}^{2}-\varphi_{1}^{2}\right)\left(x_{5}^{2}+x_{6}^{2}\right)+\left(\varphi_{3}^{2}-\right.$ $\left.\varphi_{1}^{2}\right)\left(x_{7}^{2}+x_{8}^{2}\right)$, which is $\geq-\varphi_{1}^{2} R^{2}$ provided that $\varphi_{2} \geq\left|\varphi_{3}\right| \geq \varphi_{1}>0$ and unbounded below otherwise;

36. $|\xi|^{2}=\left(\beta^{2}-\varphi_{1}^{2}\right) R^{2}+\left(\beta^{2}+\varphi_{2}^{2}-\varphi_{1}^{2}\right)\left(x_{5}^{2}+x_{6}^{2}\right)+\left(\beta^{2}+\varphi_{3}^{2}-\varphi_{1}^{2}\right)\left(x_{7}^{2}+\right.$ $\left.x_{8}^{2}\right)-4 \beta \varphi_{1}\left(x_{1} x_{3}+x_{2} x_{4}\right)$, which is unbounded below;

37. $|\xi|^{2}=\left(x_{1}+x_{4}\right)^{2}+\left(x_{2}+x_{6}\right)^{2}+\varphi^{2}\left(x_{7}^{2}+x_{8}^{2}\right)>0$;

38. $|\xi|^{2}=-\varphi_{1}^{2} R^{2}+\left(\varphi_{2}^{2}-\varphi_{1}^{2}\right)\left(x_{3}^{2}+x_{4}^{2}\right)+\left(\varphi_{3}^{2}-\varphi_{1}^{2}\right)\left(x_{5}^{2}+x_{6}^{2}\right)+\left(\varphi_{4}^{2}-\varphi_{1}^{2}\right)\left(x_{7}^{2}+x_{8}^{2}\right)$, which is $\geq-\varphi^{2} R^{2}$ for $\varphi_{2} \geq \varphi_{3} \geq \varphi_{4} \geq\left|\varphi_{1}\right|>0$; and

39. $|\xi|^{2}=\beta_{1}^{2} R^{2}+\left(\beta_{2}^{2}-\beta_{1}^{2}\right)\left(x_{2}^{2}-x_{4}^{2}\right)+\left(\varphi_{1}^{2}+\beta_{1}^{2}\right)\left(x_{5}^{2}+x_{6}^{2}\right)+\left(\varphi_{2}^{2}+\beta_{1}^{2}\right)\left(x_{7}^{2}+\right.$ $\left.x_{8}^{2}\right)$, which is $\geq \beta_{1}^{2} R^{2}$ provided that $\beta_{2}=\beta_{1}$ and unbounded below otherwise.

In this case, since we will be applying these results to the reductions of $\operatorname{AdS}_{7} \times S^{4}$ and the hair on $S^{4}$ cannot be combed, we will only be needing those Killing vectors on $\mathrm{AdS}_{7}$ whose norms are positive. From the above list we see immediately that these are (11), (25) and (39) all three with $\beta_{1}=\beta_{2}$, (23) and (37).

\section{Supersymmetry}

In this section we discuss the conditions under which a particular reduction of a Freund-Rubin background of the form $\operatorname{AdS}_{p+1} \times S^{q}$ preserves any of the (maximal) supersymmetry. This is a subtle issue, for which we have to distinguish between M-theory and supergravity. Supersymmetry in the supergravity limit is realised geometrically in terms of Killing spinors. In Mtheory this cannot be the full story. In fact, as illustrated in $[60,61,62]$ and lucidly explained more recently in [63], a background such as the FreundRubin vacuum $\mathrm{AdS}_{5} \times S^{5}$ in type IIB string theory, whose supersymmetry is fully realised geometrically, is T-dual (and hence equivalent) to a type IIA string background $\operatorname{AdS}_{5} \times \mathbb{C P}^{2} \times S^{1}$, which does not even have a spin structure.

At the supergravity level, T-duality defines a correspondence between certain backgrounds of type IIA and type IIB supergravities: two back- 
grounds being T-dual if their Kaluza-Klein reductions to $d=9 N=2$ supergravity coincide:

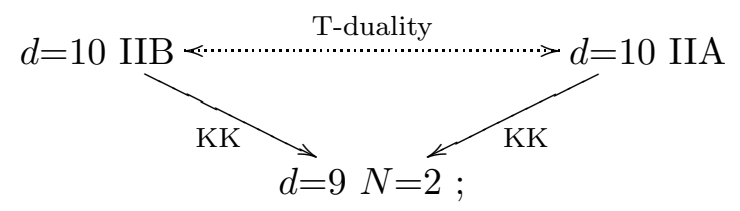

for instance,

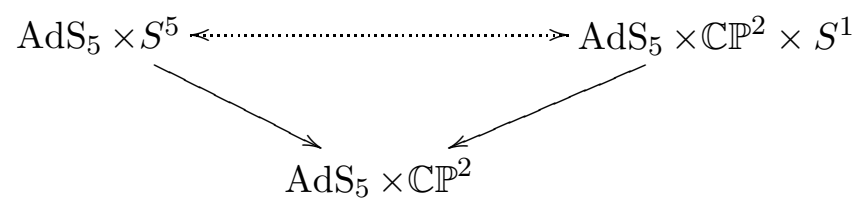

In string or M-theory we must keep all the Kaluza-Klein modes, and not just the zero modes. Supersymmetries which would appear broken from the supergravity point of view due to the non-invariance of the Killing spinors, manifest themselves among the nonzero Kaluza-Klein modes. Under Tduality these in turn become winding modes of the dual background. Indeed, the spinors in $\mathrm{AdS}_{5} \times S^{5}$ transform nontrivially under the circle subgroup along which the T-duality is being performed and hence the supersymmetry in the T-dual picture is manifested in winding modes.

From the M-theory point of view the existence of a spin structure in the quotient is therefore somewhat of a red herring, since the Killing spinors of the original background will descend in some fashion to the Kaluza-Klein reduction, even if not necessarily as spinors. From a supergravity perspective, however, it is only those Killing spinors which are invariant which do descend to Killing spinors of the reduced background and as we will see a necessary condition for the existence of invariant Killing spinors is the existence of a spin structure in the quotient.

Our primary focus in this paper being the supergravity backgrounds, we will take the point of view that when we talk about the supersymmetry preserved in a background, we will mean the geometrically realised supersymmetry of the supergravity background and hence it will be that problem which we will study. This section is thus divided into two parts. In the first part we analyse, for the geometries of interest, the problem of whether a quotient of a spin manifold is again spin. We will arrive at a criterion which can be tested by a simple calculation in a Clifford algebra. In the second part we analyse the problem of whether the background (before reduction) allows invariant Killing spinors. 


\subsection{Spin structures}

Given a Freund-Rubin background $M$, let $\Gamma$ be the one-parameter subgroup of isometries by which we are reducing. When is $M / \Gamma$ spin?

We will generalise and consider $\left(M^{1+n}, g\right)$ a simply-connected lorentzian spin manifold and $\Gamma$ a one-parameter subgroup of spacelike isometries, and ask under what conditions the quotient (assumed smooth) is spin.

Let $P_{\mathrm{SO}}(M) \rightarrow M$ denote the bundle of oriented orthonormal frames on $M$. It is a principal bundle with structure group $\mathrm{SO}(1, n)$. Since $M$ is spin and simply-connected, it has a unique spin structure $\theta: P_{\mathrm{Spin}}(M) \rightarrow$ $P_{\mathrm{SO}}(M)$. The bundle $P_{\mathrm{Spin}}(M) \rightarrow M$ is a principal bundle with group $\operatorname{Spin}(1, n)$ and the bundle map $\theta$ above is a double cover which agrees on each fibre with the standard double-cover $\theta_{0}: \operatorname{Spin}(1, n) \rightarrow \mathrm{SO}(1, n)$.

Let $\xi$ denote the Killing vector which generates the action of $\Gamma$. By assumption, it is everywhere spacelike, with norm $|\xi|>0$. Let $P \subset P_{\mathrm{SO}}(M)$ be the sub-bundle consisting of those frames which have $\xi /|\xi|$ as the first vector. This is again a principal bundle, but now with structure group $\mathrm{SO}(1, n-1)$, this being isomorphic to the isotropy subgroup of the spacelike vector $\xi /|\xi|$ at any given point.

The action of $\Gamma$ on $M$ induces an action on $P_{\mathrm{SO}}(M)$, an element $\gamma \in \Gamma$ taking an oriented orthonormal frame at the point $x \in M$ to an oriented orthonormal frame at the point $\gamma \cdot x \in M$. If the frame at $x$ is in $P$, so that it has the normalised Killing vector as the first vector, then so will be the transformed frame. This is because the Killing vector $\xi$ is $\Gamma$-invariant. The action of $\Gamma$ on $P_{\mathrm{SO}}(M)$ commutes with the natural action of $\mathrm{SO}(1, n)$, whence we have a free action of $\mathrm{SO}(1, n) \times \Gamma$ on $P_{\mathrm{SO}}(M)$. Restricting to the sub-bundle $P$ we have a free action of $\mathrm{SO}(1, n-1) \times \Gamma$. We can therefore take the quotient $P / \Gamma$ to obtain a principal $\mathrm{SO}(1, n-1)$-bundle over $M / \Gamma$. Since oriented orthonormal frames of $M / \Gamma$ are in one-to-one correspondence with oriented orthonormal frames on $M$ with $\xi /|\xi|$ as first vector, we see that indeed $P / \Gamma \rightarrow M / \Gamma$ is the bundle of oriented orthonormal frames on $M / \Gamma$.

Now consider the bundle $\theta^{-1} P \rightarrow M$, which is a sub-bundle of $P_{\text {Spin }}(M)$ with structure group $\operatorname{Spin}(1, n-1)=\theta_{0}^{-1} \mathrm{SO}(1, n-1)$. If the action of $\Gamma$ on $P_{\mathrm{SO}}(M)$ lifts to $P_{\mathrm{Spin}}(M)$ in such a way that $\theta$ is $\Gamma$-equivariant, then it preserves $\theta^{-1} P$ (by equivariance) and the quotient $\theta^{-1} P / \Gamma$ is a principal $\operatorname{Spin}(1, n-1)$ bundle over $M / \Gamma$-indeed, it is the spin lift of $P / \Gamma$. In other words, it is a spin bundle on $M / \Gamma$. Conversely, any spin bundle $P_{\text {Spin }}(M / \Gamma)$ 
on $M / \Gamma$ pulls back via the projection $\pi: M \rightarrow M / \Gamma$ to a spin bundle

$$
\pi^{*} P_{\operatorname{Spin}}(M / \Gamma) \times_{\operatorname{Spin}(1, n-1)} \operatorname{Spin}(1, n) \rightarrow M
$$

on $M$ after enlarging the structure group to $\operatorname{Spin}(1, n)$, with an equivariant $\Gamma$ action.

In other words, $M / \Gamma$ is spin if and only if the action of $\Gamma$ lifts to an action on the spin bundle $P_{\text {Spin }}(M)$. If it does, the spin bundle on $M$ is called projectable, a term introduced in [64] and discussed in more detail in [65].

There are two possible topologies for the one-parameter group $\Gamma$ : either $\mathbb{R}$ or $S^{1}$. In the former case, $\Gamma$ is simply-connected and one obtains an action of $\Gamma$ on $P_{\text {Spin }}(M)$ simply by integrating the infinitesimal action. In this case the spin bundle is always projectable. However when $\Gamma \cong S^{1}$ it may be that the infinitesimal action only lifts to an action of a double cover of $\Gamma$. Therefore the question of $M / \Gamma$ being spin only arises for reductions by circle subgroups.

If the spin bundle $P_{\text {Spin }}(M)$ is not projectable, then the action of $\Gamma$ $\left(\cong S^{1}\right)$ on the frame bundle does not lift. Instead the spin bundle admits an action of a double cover $\widetilde{\Gamma} \cong S^{1}$ of $\Gamma$ :

$$
1 \longrightarrow \mathbb{Z}_{2} \longrightarrow \widetilde{\Gamma} \stackrel{\sigma}{\longrightarrow} \Gamma \longrightarrow 1
$$

defined so that if $\tilde{p} \in P_{\text {Spin }}(M)$, then we have

$$
\theta(\tilde{\gamma} \cdot \tilde{p})=\sigma(\tilde{\gamma}) \cdot \theta(\tilde{p})
$$

for every $\tilde{\gamma} \in \widetilde{\Gamma}$. This shows that the action is free, since so is the action of $\Gamma$ on $P_{\mathrm{SO}}(M)$.

Let $P \subset P_{\mathrm{SO}}(M)$ be as above and consider the $\operatorname{Spin}(1, n-1)$-bundle $\theta^{-1} P$. Since $\Gamma$ preserves $P$, it follows from (5.1) that $\widetilde{\Gamma}$ preserves $\theta^{-1} P$.

Therefore $\theta^{-1} P$ admits a free action of $\operatorname{Spin}(1, n-1)$ on the right and a commuting free action of $\widetilde{\Gamma}$ on the left. This action is not effective, however, and there is a normal $\mathbb{Z}_{2}$ subgroup which acts trivially. To see this, notice that this subgroup must belong to the kernel of the homomorphism

$$
\operatorname{Spin}(1, n-1) \times \widetilde{\Gamma} \stackrel{\theta_{0} \times \sigma}{\longrightarrow} \operatorname{SO}(1, n-1) \times \Gamma,
$$

which is isomorphic to $\mathbb{Z}_{2} \times \mathbb{Z}_{2}$. Indeed, if $\tilde{\gamma} \in \widetilde{\Gamma}, \tilde{p} \in \theta^{-1} P$ and $\tilde{g} \in$ $\operatorname{Spin}(1, n-1)$, then

$$
\theta(\tilde{\gamma} \cdot \tilde{p} \cdot \tilde{g})=\sigma(\tilde{\gamma}) \cdot \theta(\tilde{p}) \cdot \theta_{0}(\tilde{g})
$$


whence if $\tilde{\gamma} \cdot \tilde{p} \cdot \tilde{g}=\tilde{p}$, then $\sigma(\tilde{\gamma}) \cdot \theta(\tilde{p}) \cdot \theta_{0}(\tilde{g})=\theta(\tilde{p})$, which implies that $\sigma(\tilde{\gamma})=1$ and $\theta_{0}(\tilde{g})=1$, as $\mathrm{SO}(1, n-1) \times \Gamma$ acts freely on $P$. Conversely, if $(\tilde{g}, \tilde{\gamma})$ belongs to the kernel of $\theta_{0} \times \sigma$ then $\theta(\tilde{\gamma} \cdot \tilde{p} \cdot \tilde{g})=\theta(\tilde{p})$, whence $\tilde{\gamma} \cdot \tilde{p} \cdot \tilde{g}$ and $\tilde{p}$ are in the same fibre of $\theta^{-1} P \rightarrow P$. Since $\theta$ is a double cover, this fibre consists of two points. Clearly $\mathbb{Z}_{2} \times \mathbb{Z}_{2}$, a group of order 4 , cannot act effectively in a set with two points. The kernel of this action cannot contain $(-1,1)$ or $(1,-1)$ since this would imply that either $\operatorname{Spin}(1, n-1)$ or $\widetilde{\Gamma}$ did not act effectively, which they do. On the other hand, being a two-point set, the actions of both $(-1,1)$ and $(1,-1)$ on any point $\tilde{p}$ must yield the other point $\tilde{p}^{\prime}$ in the same fibre as $\tilde{p}$; whence their product $(-1,-1)$ preserves all points $\tilde{p}$. In summary, $\theta^{-1} P$ admits a free effective action of the group

$$
\operatorname{Spin}(1, n-1)^{c}:=\operatorname{Spin}(1, n-1) \times_{\mathbb{Z}_{2}} \widetilde{\Gamma}
$$

obtained by quotienting $\operatorname{Spin}(1, n-1) \times \widetilde{\Gamma}$ by the $\mathbb{Z}_{2}$ subgroup generated by $(-1,-1)$.

In summary, the bundle $\theta^{-1} P$ can be viewed as a $\operatorname{Spin}(1, n-1)^{c}$-bundle over $M / \Gamma$; that is, it yields a $\operatorname{spin}^{c}$-structure in the quotient.

We shall not attempt here to give general conditions for a spin bundle on a manifold $M$ to yield a spin or a $\operatorname{spin}^{c}$ structure in the quotient by a circle action. Instead we shall concentrate on the geometries of interest, namely a product of space forms. Let us start with the spheres.

\subsubsection{Spin structures on sphere quotients}

The $q$-sphere $S^{q} \cong \mathrm{SO}(q+1) / \mathrm{SO}(q)$ is a homogeneous space of $\mathrm{SO}(q+1)$ and (the total space of) its oriented orthonormal frame bundle can be identified with $\mathrm{SO}(q+1)$ itself. Let $\vartheta: \operatorname{Spin}(q+1) \rightarrow \mathrm{SO}(q+1)$ be the two-fold covering map. It is a bundle map over $S^{q+1}$ and either composing these two maps or noticing that $S^{q} \cong \operatorname{Spin}(q+1) / \operatorname{Spin}(q)$ is also a homogeneous space for the Spin group, we see that $\operatorname{Spin}(q+1)$ can be identified with the total space of the spin bundle over $S^{q}$.

The usefulness of this description is that the action of isometries on the frame bundle is very natural; namely, the action of $\mathrm{SO}(q+1)$ on $S^{q}$ is induced from left multiplication on $\mathrm{SO}(q+1)$. This action is well defined on $S^{q}=\mathrm{SO}(q+1) / \mathrm{SO}(q)$ because left and right multiplications commute. Therefore the action of a subgroup $\Gamma \subset \mathrm{SO}(q+1)$ on $S^{q}$ is induced by left multiplication by $\Gamma$ itself in $\mathrm{SO}(q+1)$. A lift of this action to the spin bundle is then simply a subgroup $\widehat{\Gamma} \subset \operatorname{Spin}(q+1)$ acting on $\operatorname{Spin}(q+1)$ by left multiplication and projecting isomorphically to $\Gamma$ via $\vartheta$. In summary, 
spin structures over $S^{q} / \Gamma$ are in one-to-one correspondence with subgroups $\widehat{\Gamma} \subset \operatorname{Spin}(q+1)$ such that $\vartheta(\widehat{\Gamma})=\Gamma$ is an isomorphism. The one-parameter groups $\Gamma$ we will consider are obtained by applying the exponential map to a given line $\mathbb{R} X \subset \mathfrak{s o}(q+1)$. By embedding the Lie algebra $\mathfrak{s o}(q+1) \subset \mathrm{C} \ell(q+1)$ in the Clifford algebra and exponentiating $t X$ there, we obtain a subgroup $\widehat{\Gamma} \subset \operatorname{Spin}(q+1)$. It is then a simple matter to check whether or not $\vartheta: \widehat{\Gamma} \rightarrow \Gamma$ is an isomorphism. By construction it is a local diffeomorphism, so the question is whether $\widehat{\Gamma}$ covers $\Gamma$ precisely once.

Let us illustrate this for the complex projective spaces. Let $S^{2 n-1} \subset$ $\mathbb{C}^{n}$ be the unit sphere and let $\Gamma \subset \mathrm{SO}(2 n)$ be the circle subgroup acting diagonally on $\mathbb{C}^{n}$ via

$$
\left(z_{1}, \ldots, z_{n}\right) \mapsto\left(e^{i t} z_{1}, \ldots, e^{i t} z_{n}\right)
$$

This action is clearly free and the resulting quotient is the complex projective space $\mathbb{C P}^{n-1}$. We would like to know whether there exists a subgroup $\widehat{\Gamma} \subset$ $\operatorname{Spin}(2 n)$ isomorphic to $\Gamma$ and such that $\vartheta(\widehat{\Gamma})=\Gamma$. We will answer this by working in the Clifford algebra $\mathrm{C} \ell(2 n)$. The subgroup $\Gamma$ is generated infinitesimally by

$$
X=\Gamma_{12}+\cdots+\Gamma_{2 n-1,2 n} \in \mathfrak{s o}(2 n) \subset \mathrm{C} \ell(2 n)
$$

Exponentiating the line containing $X$ in $\mathrm{C} \ell(2 n)$ we arrive at a subgroup $\widehat{\Gamma} \subset \operatorname{Spin}(2 n) \subset \mathrm{C} \ell(2 n)$ consisting of elements

$$
\widehat{g}(t):=\exp (t / 2 X)=\left(\nVdash \cos \frac{t}{2}+\Gamma_{12} \sin \frac{t}{2}\right) \cdots\left(\nVdash \cos \frac{t}{2}+\Gamma_{2 n-1,2 n} \sin \frac{t}{2}\right),
$$

which clearly projects to $\Gamma \subset \mathrm{SO}(2 n): \widehat{g}(t) \mapsto g(t)$, where

$$
g(t)=R_{12}(t) \cdots R_{2 n-1,2 n}(t)
$$

where $R_{i j}(t)$ is the rotation by an angle $t$ in the $(i j)$-plane. The question is whether the map $\widehat{g}(t) \mapsto g(t)$ is an isomorphism. Locally it is clearly an isomorphism, but the question is whether it is one-to-one or two-to-one. Equivalently, since the pre-image of the identity in $\mathrm{SO}(2 n)$ consists of $\pm \nVdash$, the question is whether the circle $\widehat{g}(t)$ passes or not through the point $-\nVdash$. Clearly this may only happen at $t=2 \pi$ where

$$
\widehat{g}(2 \pi)=(-1)^{n} \nVdash
$$

whence $\widehat{\Gamma}$ contains $-\nVdash$ if and only if $n$ is odd. Therefore if $n$ is even, $\mathbb{C} \mathbb{P}^{n-1}$ admits a spin structure, whereas if $n$ is odd, it only admits a $\operatorname{spin}^{c}$ structure. 


\subsubsection{Spin structures on quotients of AdS}

Now we turn our attention to the AdS spaces. The situation here is complicated by the fact that the isometry group of AdS space is not a matrix group; that is, it has no faithful finite-dimensional linear representations. Recall that in this paper AdS is simply-connected, whereas the quadric $Q_{p+1} \subset \mathbb{R}^{2, p}$ is a quotient by the action of the fundamental group, which is isomorphic to $\mathbb{Z}$ for $p>2$ and to $\mathbb{Z} \oplus \mathbb{Z}$ for $p=2$. The quadric $Q_{p+1}$ is a homogeneous space for the group $\mathrm{SO}(2, p)$, whereas $\mathrm{AdS}_{p+1}$ is a homogeneous space for the group $\widetilde{\mathrm{SO}(2, p)}$ which is the central extension of $\mathrm{SO}(2, p)$ by the fundamental group of the quadric. Let $\tau \in \widehat{\mathrm{SO}(2, p)}$ denote the generator of the fundamental group of $Q_{p}+1$, for $p>2$ and let $C_{p}=\langle\tau\rangle \cong \mathbb{Z}$ be the infinite cyclic subgroup of $\widehat{\mathrm{SO}(2, p)}$ generated by $\tau$, for $p>2$. For $p=2$ we have two generators $\tau_{i} \in \widehat{\mathrm{SO}(2,2)}$ for $i=1,2$ and we let $C_{2}=\left\langle\tau_{1}, \tau_{2}\right\rangle \cong \mathbb{Z} \oplus \mathbb{Z}$ denote the abelian subgroup generated by $\tau_{1}, \tau_{2}$. Then we have the following exact sequence of groups

$$
0 \longrightarrow C_{p} \longrightarrow \widetilde{\mathrm{SO}(2, p)} \stackrel{\pi}{\longrightarrow} \mathrm{SO}(2, p) \longrightarrow 1,
$$

making $\widetilde{\mathrm{SO}(2, p)}$ into a central extension by $C_{p}$ of $\mathrm{SO}(2, p)$. The above sequence does not split; that is, there is no subgroup $\mathrm{SO}(2, p)$ of $\widetilde{\mathrm{SO}(2, p)}$ which is a section of $\pi$. Therefore $\widetilde{\mathrm{SO}(2, p)}$ is a nontrivial central extension and is thus characterised by a group 2-cocycle $\gamma$ of $\mathrm{SO}(2, p)$ with values in $C_{p}$. Group elements in $\widehat{\mathrm{SO}(2, p)}$ are given by pairs $(c, g)$ where $c \in C_{p}$ and $g \in \mathrm{SO}(2, p)$ with product

$$
\left(c_{1}, g_{1}\right)\left(c_{2}, g_{2}\right)=\left(c_{1}+c_{2}+\gamma\left(g_{1}, g_{2}\right), g_{1} g_{2}\right),
$$

where the cocycle $\gamma$ is normalised so that $\gamma(e, g)=\gamma(g, e)=0$ for $e \in$ $\mathrm{SO}(2, p)$ the identity element. Associativity of the above multiplication is equivalent to the cocycle condition for $\gamma$. It should be possible to derive an explicit formula for this cocycle in local coordinates adapted to an Iwasawa decomposition of $\mathrm{SO}(2, p)$, but we do not believe it to be particularly useful for our purposes.

The isotropy of a point of the quadric $Q_{p+1}$ is isomorphic to $\mathrm{SO}(1, p)$ and, as we will now show, the same is true for $\operatorname{AdS}_{p+1}$. Let $x \in \operatorname{AdS}_{p+1}$ be a point and let $G_{x}$ denote the corresponding isotropy subgroup of $\widehat{\mathrm{SO}(2, p)}$. Now let $\bar{x} \in Q_{p+1}$ denote the corresponding point on the quadric and $G_{\bar{x}} \subset \mathrm{SO}(2, p)$ its isotropy group. The projection $\pi: \widetilde{\mathrm{SO}(2, p)} \rightarrow \mathrm{SO}(2, p)$ restricts to a covering map $\pi: G_{x} \rightarrow G_{\bar{x}}$. We wish to show that this map is actually oneto-one. To see this notice that if $g_{1}, g_{2} \in G_{x}$ project to the same element of 
$G_{\bar{x}}$, then $g_{1} g_{2}^{-1}$ belongs to the kernel of $\pi$, which is the central subgroup $C_{p}$. However no nontrivial element of $C_{p}$ fixes the point $x$ : the fundamental group acts via deck transformations which act freely and properly discontinuous. Therefore $g_{1} g_{2}^{-1}=e$ and hence $g_{1}=g_{2}$.

This means that we have two principal $\mathrm{SO}(1, p)$ fibrations, shown by the vertical arrows of the following commutative diagram:

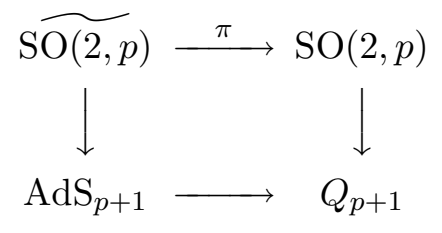

Indeed, as in the case of the sphere, these fibrations define the oriented orthonormal frame bundles of $\mathrm{AdS}_{p+1}$ and $Q_{p+1}$, respectively. Any subgroup $\Gamma \subset \widetilde{\mathrm{SO}(2, p)}$ acting on $\mathrm{AdS}_{p+1}$ acts naturally on the frame bundle by left multiplication in the group $\widetilde{\mathrm{SO}(2, p)}$.

As in the case of the sphere, the spin bundles are similarly described by composing the above fibrations with the spin double covers $\widetilde{\vartheta}: \widetilde{\operatorname{Spin}(2, p)} \rightarrow$ $\widetilde{\mathrm{SO}(2, p)}$ and $\vartheta: \mathrm{Spin}(2, p) \rightarrow \mathrm{SO}(2, p)$, yielding the following commutative diagram

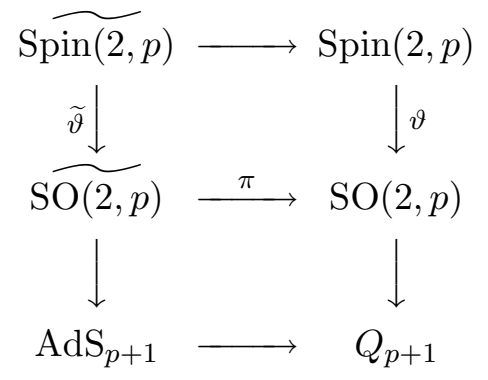

The action of a subgroup $\Gamma \subset \widetilde{\mathrm{SO}(2, p)}$ on $\mathrm{AdS}_{p+1}$ lifts to the spin bundle if and only if there is a subgroup $\widehat{\Gamma} \subset \widehat{\operatorname{Spin}(2, p)}$ such that $\widetilde{\vartheta}$ maps $\widehat{\Gamma}$ isomorphically to $\Gamma$. The difficulty in testing for the existence of such a subgroup is that, unlike $\operatorname{Spin}(2, p)$, the spin group $\widetilde{\operatorname{Spin}(2, p)}$ admits no finite-dimensional faithful representations, whence in particular it cannot be embedded in, say, a Clifford algebra. We will show, however, that the subgroups of interest in this paper are actually subgroups of $\operatorname{Spin}(2, p)$ and hence we can (and will) work with the Clifford algebra. 


\subsubsection{Spin structures on quotients of Freund-Rubin backgrounds}

Finally let us consider the product $\operatorname{AdS}_{p+1} \times S^{q}$. The oriented orthonormal frame bundle of this product, which is a principal $\mathrm{SO}(1, q+p)$ bundle, admits a reduction to an $\mathrm{SO}(1, p) \times \mathrm{SO}(q)$ bundle, whose total space is the Lie group $\widetilde{\mathrm{SO}(2, p)} \times \mathrm{SO}(q+1)$. Similarly the spin bundle admits a reduction to the subgroup of $\operatorname{Spin}(1, p+q)$ obtained as the image of $\operatorname{Spin}(1, p) \times \operatorname{Spin}(q)$ under the canonical lift (denoted by the dotted line)

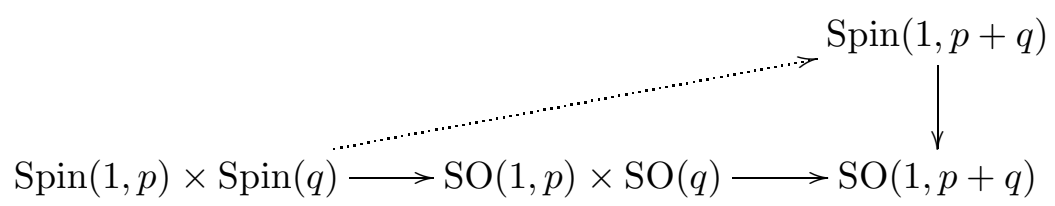

of the map obtained by composing the natural projection to $\mathrm{SO}(1, p) \times \mathrm{SO}(q)$ with the natural inclusion of this group into $\mathrm{SO}(1, p+q)$. The lift exists because $\operatorname{Spin}(1, p) \times \operatorname{Spin}(q)$ is simply-connected (for the values of $p$ and $q$ that we are interested in and restricting implicitly to the identity component) and hence any map to a space - here $\mathrm{SO}(1, p+q)$ - lifts to its universal cover. The lift (5.2) is not an embedding however; but actually factors through a $\mathbb{Z}_{2}$-quotient. This can be seen more transparently in the Clifford algebra. We can embed $\operatorname{Spin}(1, p)$ and $\operatorname{Spin}(q)$ into the Clifford algebras $\mathrm{C} \ell(1, p)$ and $\mathrm{C} \ell(q)$, respectively. The lift $(5.2)$ is then induced by the natural isomorphism $\mathrm{C} \ell(1, p) \widehat{\otimes} \mathrm{C} \ell(q) \cong \mathrm{C} \ell(1, p+q)$, where $\widehat{\otimes}$ is the $\mathbb{Z}_{2}$-graded tensor product. (This just means that the $\gamma$-matrices of $\mathrm{C} \ell(1, p)$ anticommute with those of $\mathrm{C} \ell(q)$.) Under this embedding we see that the element $(-\nVdash,-\nVdash)$ in $\operatorname{Spin}(1, p) \times \operatorname{Spin}(q)$ gets mapped to the identity in $\operatorname{Spin}(1, p+q)$. Therefore the lift (5.2) factors through the group

$$
\operatorname{Spin}(1, p) \times_{\mathbb{Z}_{2}} \operatorname{Spin}(q)
$$

where $\mathbb{Z}_{2}$ is the subgroup generated by $(-\nVdash,-\nVdash)$.

The total space of the reduction of the spin bundle to the above group is then precisely the quotient of $\widehat{\operatorname{Spin}(2, p)} \times \operatorname{Spin}(q+1)$ by the $\mathbb{Z}_{2}$-subgroup of $\operatorname{Spin}(1, p) \times \operatorname{Spin}(q)$ generated by $(-\nVdash,-\nVdash)$, now embedded in $\widehat{\operatorname{Spin}(2, p)} \times$ $\operatorname{Spin}(q+1)$.

A subgroup $\Gamma \subset \widetilde{\mathrm{SO}(2, p)} \times \mathrm{SO}(q+1)$ acts on the frame bundle by left multiplication and its action lifts to the spin bundle if there is a subgroup $\widehat{\Gamma} \subset \widehat{\operatorname{Spin}(2, p)} \times_{\mathbb{Z}_{2}} \operatorname{Spin}(q+1)$ which projects isomorphically to $\Gamma$ under the spin covering map

$$
\widetilde{\operatorname{Spin}(2, p)} \times_{\mathbb{Z}_{2}} \operatorname{Spin}(q+1) \longrightarrow \widetilde{\mathrm{SO}(2, p)} \times \mathrm{SO}(q+1),
$$


which is a double cover.

We are interested in one-parameter subgroups $\Gamma$ and these are obtained by exponentiating a one-dimensional subspace of the Lie algebra. There are two possible topologies for $\Gamma$ : either the real line or the circle. If $\Gamma$ is diffeomorphic to the real line, then the lift $\widehat{\Gamma}$ is also diffeomorphic to the real line, since it has to be a connected cover of the real line, which is simplyconnected; hence $\widehat{\Gamma} \cong \Gamma$. Only when $\Gamma$ is diffeomorphic to the circle, can we have an obstruction.

Circle subgroups of $\widetilde{\mathrm{SO}(2, p)} \times \mathrm{SO}(q+1)$ are contained in a maximal compact subgroup, which is isomorphic to $\mathrm{SO}(p) \times \mathrm{SO}(q+1)$. Moreover, the $\mathrm{SO}(p)$ component is contained in the isotropy $\mathrm{SO}(1, p)$ of a point in $\mathrm{AdS}_{p+1}$, which as we showed above projects isomorphically to the isotropy of a point in $Q_{p+1}$. In other words, the maximal compact subgroups $\mathrm{SO}(p) \times \mathrm{SO}(q+1)$ are diffeomorphic to their image under the projection $\pi$ and this means that we can effectively work in the quotient group $\mathrm{SO}(2, p) \times \mathrm{SO}(q+1)$. This simplifies the analysis considerably because $\operatorname{Spin}(2, p) \times_{\mathbb{Z}_{2}} \operatorname{Spin}(q+1)$ can be embedded in the Clifford algebra $\mathrm{C} \ell(2, p+q+1)$. A circle subgroup $\Gamma \subset \mathrm{SO}(2, p) \times \mathrm{SO}(q+1)$ is obtained by exponentiating a line $\mathbb{R} X \subset \mathfrak{s o}(2, p) \oplus$ $\mathfrak{s o}(q+1)$ in the Lie algebra, and exponentiating this same line in the Clifford algebra $\mathrm{C} \ell(2, p+q+1)$ yields a circle subgroup $\widehat{\Gamma} \subset \operatorname{Spin}(2, p) \times_{\mathbb{Z}_{2}} \operatorname{Spin}(q+1)$, which covers $\Gamma$. We must determine if $\widehat{\Gamma}$ is isomorphic to $\Gamma$ or if, on the contrary, it is a double cover. This will only be the case if $\widehat{\Gamma}$ contains the point other than the identity in $\operatorname{Spin}(2, p) \times_{\mathbb{Z}_{2}} \operatorname{Spin}(q+1)$ which projects to the identity in $\mathrm{SO}(2, p) \times \mathrm{SO}(q+1)$; equivalently, if $\widehat{\Gamma} \subset \mathrm{C} \ell(2, p+q+1)$ contains the point $-\nVdash$. This can then be checked in the same way as was illustrated earlier for the complex projective spaces.

\section{$5.2 \quad$ Killing spinors}

Let $S$ denote the representation of $\operatorname{Spin}(1, n)$ corresponding to the spinors in the supergravity theory on $M$. The spinor bundle associated to this representation is defined to be

$$
S(M):=P_{\operatorname{Spin}}(M) \times_{\operatorname{Spin}(1, n)} S .
$$

If the spin bundle is projectable, then $\Gamma$ acts naturally on $S(M)$, since it acts on $P_{\text {Spin }}(M)$ and commutes with the action of $\operatorname{Spin}(1, n)$. The supergravity Killing spinors on $M$ are sections of $S(M)$ which are parallel with respect to a connection

$$
D: S(M) \rightarrow T^{*} M \otimes S(M)
$$


which is $\Gamma$-invariant because $D$ depends on the bosonic fields of the background which are left invariant by the action of $\Gamma$. This means that the space of Killing spinors is a representation of $\Gamma$. The $\Gamma$-invariant Killing spinors are the Killing spinors of the quotient.

Let us consider the case of a circle action, for definiteness. Associated to the principal circle bundle $M \rightarrow M / \Gamma$ there is a complex line bundle

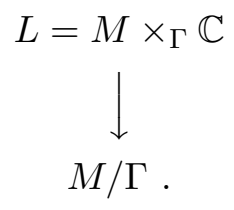

This line bundle is equipped with a natural connection coming from the connection on $M \rightarrow M / \Gamma$ which declares those vectors orthogonal to the Killing vector $\xi$ as horizontal. In the case where $M$ is a background of elevendimensional supergravity, the connection one-form on the IIA background $M / \Gamma$ is the Ramond-Ramond one-form potential.

Sections of $L$ are identified with the first Kaluza-Klein mode of a complex valued function $f: M \rightarrow \mathbb{C}$. In other words, a function which obeys $\xi f=$ if. More generally, any function $f: M \rightarrow \mathbb{C}$ admits a decomposition into Kaluza-Klein modes

$$
f=\sum_{n \in \mathbb{Z}} f_{n}
$$

where $\xi f_{n}=i n f_{n}$. This is nothing but the Fourier decomposition of the function $f$ along the orbits of $\Gamma$. From the point of view of the quotient, $f_{n}$ defines a section through $L^{n}$, where $L^{-1}=L^{*}$ is the dual bundle and $L^{0}$ is the trivial bundle. Similarly we can decompose a complex spinor $\psi$ into its Kaluza-Klein modes

$$
\psi=\sum_{n \in \mathbb{Z}} \psi_{n}
$$

where $\mathcal{L}_{\xi} \psi_{n}=i n \psi_{n}$, with $\mathcal{L}_{\xi}$ the spinorial Lie derivative. From the point of view of the quotient, $\psi_{n}$ is a section of the bundle $S(M / \Gamma) \otimes L^{n}$, where $S(M / \Gamma)$ is the bundle $\theta^{-1} P \times_{\operatorname{Spin}(1, n-1)} S$. The Kaluza-Klein zero modes are spinors of $M / \Gamma$, whereas the nonzero modes are the 'charged spinors' of [63].

The covariant derivative $D$ defining the Killing spinors commutes with the action of $\Gamma$, whence it preserves the Fourier decomposition. This means that we will be able to write the (complexified) Killing spinors as a finite linear combination of Fourier modes. The zero modes will be Killing spinors on the quotient, which correspond to the geometrically realised supersymmetries. It is not clear to us whether there is any supergravity interpretation 
of the nonzero modes. In M/string-theory and under T-duality, these modes become winding (or brane) modes in the dual background and are the ones responsible for the 'supersymmetry without supersymmetry' of [60].

If the spin bundle on $M$ is not projectable we have a similar situation with the difference that the infinitesimal action of $\xi$ on spinors integrates to an action of the double cover $\widetilde{\Gamma}$. In terms of the eigenvalues of $\mathcal{L}_{\xi}$ this means that they are now $i \lambda$ with $\lambda \in \mathbb{Z}+\frac{1}{2}$. It may be surprising at first that there are no integral eigenvalues - after all this is still a representation of $\widetilde{\Gamma}$, albeit one with a kernel. The reason is topological. A spinor on $M$ with eigenvalue in with $n \in \mathbb{Z}$ defines a section through a bundle $S^{\prime} \otimes L^{n}$, where $S^{\prime}$ would be a spinor bundle on $M / \Gamma$, but this bundle does not exist. Instead a spinor with eigenvalue $i\left(n+\frac{1}{2}\right)$ defines a section through $\left(S^{\prime} \otimes L^{1 / 2}\right) \otimes L^{n}$, where the bundles $S^{\prime}$ and $L^{1 / 2}$ do not exist individually but their tensor product $S^{c}(M / \Gamma)=S^{\prime} \otimes L^{1 / 2}$ does-indeed, it is the bundle of $\operatorname{spin}^{c}$ spinors, as the notation suggests. Killing spinors on $M$ decompose into Fourier modes to give rise to sections of $S^{c}(M / \Gamma) \otimes L^{n}$ for some $n$. We see therefore that even when $n=0$, the sections of $S^{c}(M / \Gamma)$ carry (fractional) charge under $\Gamma$, and hence there are no zero modes. The supergravity interpretation of these charged spinors is also not clear, although some speculations are presented in the paper [63].

These remarks give another way to test for the existence of a spin structure in the quotient in the case of a circle action, which is the only case where there is a possible obstruction in the groups we are considering. We simply analyse the representation of the circle group (normalised to $\mathbb{R} / 2 \pi \mathbb{Z}$ ) on the Killing spinors and check whether the charges are integral, in which case there is a spin structure in the quotient, or half-integral, in which case there is not and only a $\operatorname{spin}^{c}$ structure.

It should be clear that this analysis requires determining the action of isometries on the Killing spinors of the backgrounds in question. It is not always appreciated that this question can be answered without ever having to compute a Killing spinor explicitly and, although doing so might provide a concrete check, the problem is simply one of group theory. The upshot of the analysis, to be presented in detail in [56] as part of a general study of smooth Freund-Rubin backgrounds, is that Killing spinors on $\operatorname{AdS}_{p+1} \times S^{q}$ are in one-to-one correspondence with the tensor product of half-spin representations of $\operatorname{Spin}(2, p)$ and $\operatorname{Spin}(q+1)$ (and possibly the R-symmetry group) and that this correspondence is equivariant with respect to the action of the isometry group [57] and the R-symmetry group.

Let us be more precise. As a representation of the symmetry (i.e., isom- 
etry $\times$ R-symmetry) group, the space of Killing spinors of the different Freund-Rubin vacua considered in this paper is isomorphic to the ones in Table 5 . Let us explain the notation in the table. The notation $\Delta^{m, n}$ stands for the spinorial representation of $\operatorname{Spin}(m, n)$, and $\Delta_{ \pm}^{m, n}$ for the chiral spinor representations, whenever they exist. Therefore, $\Delta_{-}^{2,2}$ is the real 2-dimensional representation of $\operatorname{Spin}(2,2)$ consisting of negative chirality spinors, whereas $\Delta_{-}^{4,0}$ denotes the negative chirality spinor representation of $\operatorname{Spin}(4)$, which is quaternionic of (complex) dimension 2. $\Delta$ is the fundamental representation of $\operatorname{Sp}(1)$, which is also quaternionic and of (complex) dimension $2 . \Delta^{2,3}$ is the 4-dimensional spinorial representation of $\operatorname{Spin}(2,3)$ and $\Delta_{-}^{8,0}$ is one of the two 8-dimensional spinorial representations of $\operatorname{Spin}(8)$. Both representations are real. Similarly, $\Delta^{5,0}$ is the fundamental representation of $\operatorname{Sp}(2) \cong \operatorname{Spin}(5)$, whence it is quaternionic and of complex dimension $4 . \Delta_{-}^{2,6}$, which is also quaternionic but of complex dimension 8 , is one of the two spinorial representations of $\operatorname{Spin}(2,6)$. The tensor product of two quaternionic representations has a real structure and the notation $[R]$ denotes the underlying real representation. Finally, $\Delta^{2,4}$ is one of the 4-dimensional complex spinor representations of $\operatorname{Spin}(2,4)$ and $\Delta^{6,0}$ is the fundamental representation of $\mathrm{SU}(4) \cong \operatorname{Spin}(6)$, which is also complex and 4-dimensional. The notation $\llbracket R \rrbracket$ means the underlying real representation of $R \oplus \bar{R}$, which has a natural real structure. We remind the reader that $\operatorname{dim}_{\mathbb{R}}[R]=\operatorname{dim}_{\mathbb{C}} R$ whereas $\operatorname{dim}_{\mathbb{R}} \llbracket R \rrbracket=2 \operatorname{dim}_{\mathbb{C}} R$.

\begin{tabular}{|c|l|l|}
\hline Vacuum & \multicolumn{1}{|c|}{ Symmetry group } & \multicolumn{1}{|c|}{ Killing spinors } \\
\hline \hline $\operatorname{AdS}_{3} \times S^{3}$ & $\operatorname{Spin}(2,2) \times \operatorname{Spin}(4) \times \operatorname{Sp}(1)$ & $\Delta_{-}^{2,2} \otimes\left[\Delta_{-}^{4,0} \otimes \Delta\right]$ \\
$\operatorname{AdS}_{4} \times S^{7}$ & $\operatorname{Spin}(2,3) \times \operatorname{Spin}(8)$ & $\Delta^{2,3} \otimes \Delta_{-}^{8,0}$ \\
$\operatorname{AdS}_{5} \times S^{5}$ & $\operatorname{Spin}(2,4) \times \operatorname{Spin}(6)$ & $\llbracket \Delta^{2,4} \otimes \Delta^{6,0} \rrbracket$ \\
$\operatorname{AdS}_{7} \times S^{4}$ & $\operatorname{Spin}(2,6) \times \operatorname{Spin}(5)$ & {$\left[\Delta_{-}^{2,6} \otimes \Delta^{5,0}\right]$} \\
\hline
\end{tabular}

Table 5: Killing spinors of Freund-Rubin vacua as representations of the symmetry group. (See the main text for an explanation of the notation.)

\section{Supersymmetric quotients of Freund-Rubin back- grounds}

In this section we bring together the technology developed in the previous sections and classify the supersymmetric smooth $S^{1}$ or $\mathbb{R}$ quotients of the 
maximally supersymmetric Freund-Rubin vacua of type IIB superstring and M-theory, as well as (the trivial lift to IIB supergravity of) the Freund-Rubin vacuum of $(1,0)$ six-dimensional supergravity. The discrete version of these and other quotients will be studied in more detail in a separate publication [41].

To this end we would like to classify all the (connected) one-parameter subgroups $\Gamma \subset \mathrm{SO}(2, p) \times \mathrm{SO}(q+1)$ such that $\left(\operatorname{AdS}_{p+1} \times S^{q}\right) / \Gamma$ is a smooth supersymmetric background of the relevant supergravity theory in $p+q+1$ dimensions, for $(p, q)=(2,3),(3,7),(4,5),(6,4)$. Since $\Gamma$ is generated by a Killing vector $\xi$, these conditions on the quotient become conditions on $\xi$ and on its integral curves. First of all, we demand that $\xi$ be everywhere spacelike: this implies in particular that it never vanishes, whence the action of $\Gamma$ is locally free. For the quotient to be smooth, we demand in addition that every point should have trivial stabiliser. For the quotient to be a supergravity background we require that it be spin, which as discussed in the previous section requires the spin structure in $\mathrm{AdS}_{p+1} \times S^{q}$ to be projectable, so that it is acted upon by $\Gamma$. Finally, supersymmetry requires that some of the Killing vectors in $\mathrm{AdS}_{p+1} \times S^{q}$ should be $\Gamma$-invariant.

Following the discussion in $[1,24,25]$, the amount of supersymmetry preserved by the reduction by a one-parameter subgroup $\Gamma$ of isometries generated by a Killing vector $\xi$ is the dimension of the vector space of solutions to the equation

$$
\mathcal{L}_{\xi} \varepsilon=\nabla_{\xi} \varepsilon+\frac{1}{4} d \xi^{b} \cdot \varepsilon=0
$$

where $\varepsilon$ is a Killing spinor and $\mathcal{L}_{\xi}$ is the Lie derivative operator along the Killing vector field $\xi$ introduced in [66]. For the backgrounds under consideration, this is simply the action of the element $\xi$ in the Lie algebra of the isometry group on the relevant spinorial representation. These representations appear in Table 5 and the action of $\xi$ on them follows in many cases by a simple weight analysis. The method has been explained in detail in [24, Appendix A] and we will simply apply it here to the cases at hand.

Finally, we stress again that we shall not discuss whether the everywhere spacelike quotients we classify have closed causal curves, even though we are aware that some of them do. Such considerations will appear in the forthcoming paper [41]. 


\subsection{Supersymmetric quotients of $\mathrm{AdS}_{3} \times S^{3}\left(\times \mathbb{R}^{4}\right)$}

The $\mathrm{AdS}_{3} \times S^{3}$ vacuum of six-dimensional $(1,0)$ supergravity corresponds geometrically to a simply-connected parallelised Lie group: $\widetilde{\mathrm{SL}}(2, \mathbb{R}) \times \mathrm{SU}(2)$, where $\widetilde{\mathrm{SL}}(2, \mathbb{R})$ is the universal covering group of $\mathrm{SL}(2, \mathbb{R})$. Both factors have equal radii of curvature, whence their scalar curvatures have equal magnitude but opposite sign. Letting $s>0$ denote the scalar curvature of the sphere, the 3 -form is then given by

$$
H=\sqrt{\frac{2}{3} s}\left(\operatorname{dvol}_{A}-\operatorname{dvol}_{S}\right)
$$

where $\operatorname{dvol}_{A}$ and $\mathrm{dvol}_{S}$ are the volume forms of $\mathrm{AdS}_{3}$ and $S^{3}$, respectively. This vacuum solution has eight supersymmetries. It is also a vacuum solution of the $(2,0)$ supergravity, this time with 16 supersymmetries [67]. We remark here, once and for all, that in this and other Freund-Rubin backgrounds we will study in this paper, the volume forms $\mathrm{dvol}_{A}$ and/or $\mathrm{dvol}_{S}$ are the volume forms of the actual metrics which appear in the supergravity solution. In particular, they are not normalised to unit radius of curvature.

This background can be lifted to IIB supergravity giving rise to nondilatonic backgrounds of the form $\mathrm{AdS}_{3} \times S^{3} \times X$, where supersymmetry implies that $X$ admits parallel spinors. We will focus on the simply-connected half-BPS background where $X=\mathbb{R}^{4}$. The space of Killing spinors for this background is isomorphic to

$$
\left(\Delta_{+}^{2,2} \otimes\left[\Delta_{+}^{4,0} \otimes \Delta_{+}^{0,4}\right]\right) \oplus\left(\Delta_{-}^{2,2} \otimes\left[\Delta_{-}^{4,0} \otimes \Delta_{+}^{0,4}\right]\right)
$$

as a representation of $\operatorname{Spin}(2,2) \times \operatorname{Spin}(4) \times \operatorname{Spin}(4)$, in the notation introduced and explained at the end of the previous section. We will moreover restrict our attention to quotients of this IIB background by one-parameter subgroups of the isometries of the $\mathrm{AdS}_{3} \times S^{3}$ geometry. The general case can be treated along similar lines without any additional difficulty, but it falls somewhat outside the scope of this paper thematically. Restricting to these quotients has the added virtue that we can then simply read off the supersymmetric quotients of the $\operatorname{AdS}_{3} \times S^{3}$ vacuum of $(1,0)$ supergravity in six dimensions, whose Killing spinors are in one-to-one correspondence with the second factor in the above decomposition, after identifying the $\operatorname{Sp}(1) \subset \operatorname{Spin}(4)$ subgroup acting nontrivially on $\Delta_{+}^{0,4}$ with the R-symmetry group of the six-dimensional theory. Similarly we can work in $(2,0)$ supergravity by substituting $\operatorname{Sp}(1)$ for $\operatorname{Sp}(2)$ and essentially doubling the number of supersymmetries. This means that the supersymmetry fraction of the quotient is the same for $(1,0)$ and $(2,0)$ supergravities. 
The Killing vector $\xi$ generating the action of $\Gamma$ decomposes as

$$
\xi=\xi_{A}+\xi_{S},
$$

where $\xi_{A}$ is the component on $\mathrm{AdS}_{3}$ and $\xi_{S}$ is the component on $S^{3}$. Moreover its norm is the sum of the norms of these two components.

Let $S^{3}$ denote the sphere of radius $R_{S}$ in $\mathbb{R}^{4}$. Up to conjugation by $\mathrm{SO}(4)$, the most general Killing vector can be written as

$$
\xi_{S}=\theta_{1} R_{12}+\theta_{2} R_{34},
$$

where $\theta_{i}$ are real parameters and where $R_{i j}$ is the infinitesimal generator of rotations in the $i j$-plane. Strictly speaking we still have to quotient by the Weyl group, which acts on the $\theta_{i}$ by permuting them and changing both their signs simultaneously. This means that we can always arrange the $\theta_{i}$ in such a way that $\theta_{1} \geq\left|\theta_{2}\right|$. We will take this ordering to hold from now on. Notice that $\theta_{2}$ need not be non-negative.

The norm of the Killing vector $\xi_{S}$ is bounded both above and below in the sphere:

$$
\theta_{2}^{2} R_{S}^{2} \leq\left|\xi_{S}\right|^{2} \leq \theta_{1}^{2} R_{S}^{2}
$$

It is not hard to see that these bounds are sharp. As a consequence, the norm of $\xi=\xi_{A}+\xi_{S}$ obeys the bounds

$$
|\xi|^{2} \geq\left|\xi_{A}\right|^{2}+\theta_{2}^{2} R_{S}^{2}
$$

whence $\xi$ will be always spacelike provided that

$$
\left|\xi_{A}\right|^{2}>-\theta_{2}^{2} R_{S}^{2}
$$

From the results in Section 4.1, the only Killing vectors $\xi_{A}$ on $\mathrm{AdS}_{3}$ which satisfy this condition without any requirement are the ones labelled (4), (5), (6) and (11) with $\beta_{1}= \pm \beta_{2}$. Recall that for this vacuum solution, $R_{S}=R_{A}=R$. Thus, the cases labelled (8) and (10), which are bounded from below whenever $\varphi>0$ and $\varphi_{1}^{2} \geq \varphi_{2}^{2}$, respectively, will also satisfy the above condition if $\theta_{2}^{2}>\varphi^{2}$ and $\theta_{2}^{2}>\varphi_{2}^{2}$, respectively. The resulting Killing vectors are everywhere spacelike with norms bounded below by $\theta_{2}^{2} R^{2}$ for cases (4), (5) and (6); by $\left(\theta_{2}^{2}+\beta_{1}^{2}\right) R^{2}$ for case (11); by $\left(\theta_{2}^{2}-\varphi^{2}\right) R^{2}(\varphi>0)$ for case (8); and by $\left(\theta_{2}^{2}-\varphi_{2}^{2}\right) R^{2}$ (where $\varphi_{1}^{2} \geq \varphi_{2}^{2}$ ) for case (10). We now proceed to analyse each case in more detail.

Notice that in principle given a Killing vector field $\xi$, what we have is a pencil of Killing vectors: a linear combination of a Killing vector on $\mathrm{AdS}_{3}$ and a Killing vector on $S^{3}$ which is then projectivised. This means that one or the 
other of the two Killing vectors (but not both) may vanish. However since we are only interested in those Killing vectors being spacelike everywhere, and the norm of $\xi_{S}$ is bounded from above, it is clear that we shall only consider the subset of $\xi_{A}$ being bounded from below. In particular, the sphere component must always be present in cases (4), (5), (6), (8) and (10). As explained above, in cases (8) and (10) even the infinitesimal parameters in $\xi_{S}$ can no longer be arbitrary but have to satisfy some requirements. On the other hand, this sphere component may be absent in case (11) with $\beta_{1}= \pm \beta_{2}$. We start by analysing the case when only the spherical component is present.

\subsection{1 $\xi=\xi_{S}$}

For $\xi$ to be everywhere nonvanishing (and hence spacelike) we require $\theta_{2}>0$. This guarantees that the action of $\Gamma$ is locally free. To have a smooth quotient, the stabilisers must be trivial. To check when this is the case it is convenient to think of $S^{3}$ as embedded in $\mathbb{C}^{2}$ with standard coordinates $z_{i}$, $a=1,2$ as the quadric

$$
\left|z_{1}\right|^{2}+\left|z_{2}\right|^{2}=R^{2}
$$

The action of $\xi$ integrates to

$$
\left(z_{1}, z_{2}\right) \mapsto\left(e^{i \theta_{1} t} z_{1}, e^{i \theta_{2} t} z_{2}\right)
$$

The quotient will not be Hausdorff unless the integral curves are periodic (compare with the discussion in [25, Section 3.2]), whence there is a smallest positive number $T$ for which

$$
\left(e^{i \theta_{1} T} z_{1}, e^{i \theta_{2} T} z_{2}\right)=\left(z_{1}, z_{2}\right)
$$

for all $\left(z_{1}, z_{2}\right)$. This implies that there are integers $p_{i}$ such that $\theta_{i} T=2 \pi p_{i}$ and since $T$ is the smallest such positive integer, the $p_{i}$ are coprime. Using the freedom to rescale the Killing vector, we can take

$$
\xi=p_{1} R_{12}+p_{2} R_{34} \quad \text { with } \operatorname{gcd}\left(p_{1}, p_{2}\right)=1,
$$

which integrates to a circle action

$$
\left(z_{1}, z_{2}\right) \mapsto\left(e^{i p_{1} t} z_{1}, e^{i p_{2} t} z_{2}\right)
$$

with period $2 \pi$. The action has trivial stabilisers if for all $\left(z_{1}, z_{2}\right)$ obeying (6.3), the equation

$$
\left(z_{1}, z_{2}\right)=\left(e^{i p_{1} t} z_{1}, e^{i p_{2} t} z_{2}\right)
$$


holds only for $t \in 2 \pi \mathbb{Z}$. By looking at the points $(2 R, 0),(0,2 R)$, we see that $p_{i}$ have to be \pm 1 giving $2^{2}=4$ possibilities which, taking into account the action of the Weyl group, are reduced to only two: $p_{1}=1$ and $p_{2}= \pm 1$. In either case the resulting quotient is $\mathbb{C P}^{1}$ (with a different orientation for each quotient), which as we saw above, admits a spin structure.

Let us analyse whether supersymmetry is preserved when taking this quotient. As discussed above, the Killing spinors of this background are in a representation $(6.2)$ of $\operatorname{Spin}(2,2) \times \operatorname{Spin}(4) \times \operatorname{Spin}(4)$. Complexifying this representation and computing the weights of the element in the first $\mathfrak{s o}(4)$ corresponding to $\xi^{ \pm}=R_{12} \pm R_{34}$, we see that

$$
\Delta_{ \pm}^{2,2} \otimes \Delta_{ \pm}^{4,0} \otimes \Delta_{+}^{0,4}
$$

has all eight weights equal to 0 for $\xi^{ \pm}$, and has weights $(1,-1)$, each with multiplicity 4 , for $\xi^{\mp}$. We conclude that either quotient preserves one half of the sixteen supersymmetries of the original background. In other words, these quotients have eight supersymmetries, or equivalently, they preserve a fraction $\nu=\frac{1}{4}$ of the IIB supersymmetry.

As backgrounds of $(1,0)$ and $(2,0) d=6$ supergravities, the quotient by $\xi^{+}$breaks all supersymmetry, whereas the one by $\xi^{-}$preserves all. The existence of nontrivial quotients preserving all supersymmetry was explained in [67], and is intimately linked to the fact that these vacua can be interpreted as anti-selfdual lorentzian Lie groups. The resulting five-dimensional backgrounds were also classified in [67] and further elucidated in [50].

We now turn our attention to the four possible families of reductions where the anti-de Sitter component of the Killing vector is nonzero.

\subsection{2 $\xi=\xi_{A}^{(4)}+\xi_{S}$}

This case is very similar to the previous one, with the possibility of adding a spatial rotation $\xi_{A}^{(4)}=\varphi \boldsymbol{e}_{34}$ to $\xi$. The same analysis as before shows that $\varphi T=2 \pi n$ for some integer $n$ which is coprime to the $p_{i}$, whence after rescaling the Killing vector it becomes

$$
\xi=n \boldsymbol{e}_{34}+p_{1} R_{12}+p_{2} R_{34} \quad \text { with } \operatorname{gcd}\left(n, p_{1}, p_{2}\right)=1
$$

As before, the triviality of the stabilisers demands that $p_{i}= \pm 1$, and hence up to rescaling $\xi$ and conjugating by the Weyl group of $\mathrm{SO}(4)$ one has

$$
\xi^{ \pm}=n \boldsymbol{e}_{34}+R_{12} \pm R_{34} \quad \text { with } n=1,2, \ldots
$$


Let us employ the convention that $\gamma_{i}$ denote the $\gamma$-matrices for $\mathrm{C} \ell(2,2)$ and $\Gamma_{a}$ denote those of $\mathrm{C} \ell(4)$. Then the element in $\mathfrak{s o}(2,2) \oplus \mathfrak{s o}(4) \subset \mathrm{C} \ell(2,6)$ corresponding to $\xi^{ \pm}$is given by

$$
X^{ \pm}=n \gamma_{34}+\Gamma_{12} \pm \Gamma_{34}
$$

Exponentiating this element we get

$$
\begin{aligned}
\widehat{g}(t)=\exp \frac{t X^{ \pm}}{2}=\left(\nVdash \cos \frac{n t}{2}+\gamma_{34} \sin \frac{n t}{2}\right)(\nVdash & \left.\cos \frac{t}{2}+\Gamma_{12} \sin \frac{t}{2}\right) \\
& \times\left(\nVdash \cos \frac{t}{2} \pm \Gamma_{34} \sin \frac{t}{2}\right)
\end{aligned}
$$

and this can only possibly equal $-\nVdash$ at $t=2 \pi$, where

$$
\widehat{g}(2 \pi)=(-1)^{n} \nVdash ;
$$

whence only for $n$ even do we have a spin structure in the quotient.

Computing the weights under $\xi^{ \pm}$of the (complexified) Killing spinors we obtain from

$$
\Delta_{+}^{2,2} \otimes \Delta_{+}^{4,0} \otimes \Delta_{+}^{0,4}
$$

$\pm \frac{n}{2}$, each with multiplicity 4 , for $\xi^{+}$and $\pm \frac{n}{2} \pm 1$ with uncorrelated signs, each with multiplicity 2 , for $\xi^{-}$; whereas from

$$
\Delta_{-}^{2,2} \otimes \Delta_{-}^{4,0} \otimes \Delta_{+}^{0,4}
$$

$\pm \frac{n}{2} \pm 1$ with uncorrelated signs, each with multiplicity 2 , for $\xi^{+}$and $\pm \frac{n}{2}$, each with multiplicity 4 , for $\xi^{-}$. Since $n$ is positive and even, we see that we only have zero weights when $n=2$, in which case there are four for either quotient. Therefore the quotient preserves one fourth of the supersymmetry of the background, or a fraction $\nu=\frac{1}{8}$ of the IIB supersymmetry.

As six-dimensional supergravity backgrounds, the $\xi^{+}$quotient is a halfBPS background for $(1,0)$ and $(2,0)$ supergravities, whereas the $\xi^{-}$quotient is not supersymmetric.

\subsection{3 $\xi=\xi_{A}^{(5)}+\xi_{S}$}

Here the Killing vector field is

$$
\xi=e_{13}-e_{34}+\theta_{1} R_{12}+\theta_{2} R_{34} .
$$

This vector fields integrates to the action of $\Gamma \cong \mathbb{R}$. Its orbits are generically non-periodic, except at those points where the action of $\xi_{A}$ vanishes, that 
is, at $x_{1}=x_{3}=x_{4}=0$, which corresponds to the two points $x_{2}= \pm R$, where we must be more careful. In these points, the orbits are either not periodic, in which case they would be dense in a submanifold of dimension at least 2 and hence the quotient would not be Hausdorff, or else they are periodic in which case their points have nontrivial stabiliser, namely the subgroup $\Gamma_{0} \subset \Gamma$ consisting of the periods. In either case the quotient is not smooth. Nevertheless see Section 7.2.2 for a discussion on how such a singular quotient can still preserve some supersymmetry.

\subsection{4 $\xi=\xi_{A}^{(6)}+\xi_{S}$}

In this case the Killing vector is

$$
\xi^{ \pm}=\mp \boldsymbol{e}_{12}-\boldsymbol{e}_{13} \pm \boldsymbol{e}_{24}+\boldsymbol{e}_{34}+\theta_{1} R_{12}+\theta_{2} R_{34},
$$

where the $\theta_{i}$ are nonzero for $\xi$ to be everywhere spacelike. Notice that the anti-de Sitter component is never zero, although its norm vanishes. It integrates to the following action on $\mathbb{R}^{2,2}$ :

$$
\left(\begin{array}{l}
x^{1} \\
x^{2} \\
x^{3} \\
x^{4}
\end{array}\right) \mapsto\left(\begin{array}{l}
x^{1}+t\left(x^{3} \mp x^{2}\right) \\
x^{2} \pm t\left(x^{1}-x^{4}\right) \\
x^{3}+t\left(x^{1}-x^{4}\right) \\
x^{4}+t\left(x^{3} \mp x^{2}\right)
\end{array}\right)
$$

whose orbits are straight lines ruling the quadric. Thus the combined Killing vector $\xi^{ \pm}$integrates to an action of $\Gamma \cong \mathbb{R}$. It is easy to see that it has trivial stabilisers, since it does on $\mathrm{AdS}_{3}$. Therefore the resulting quotient is smooth and, since $\Gamma \cong \mathbb{R}$, also spin.

To determine the amount of preserved supersymmetry, we have to solve the algebraic equation (6.1) for this particular Killing vector field $\xi$. The action of $\xi$ on the Killing spinors defines an algebraic operator which splits into a nilpotent piece

$$
N^{ \pm}=\mp \gamma_{12}-\gamma_{13} \pm \gamma_{24}+\gamma_{34}
$$

and a semisimple piece

$$
S=\theta_{1} \Gamma_{12}+\theta_{2} \Gamma_{34},
$$

which moreover commute with each other. Therefore the equation (6.1) splits into two:

$$
N^{ \pm} \varepsilon=0 \quad \text { and } \quad S \varepsilon=0
$$


Thus, the invariant Killing spinors correspond to the intersection of the kernels of $N^{ \pm}$and $S$ acting on the space

$$
\left(\Delta_{+}^{2,2} \otimes \Delta_{+}^{4,0} \otimes \Delta_{+}^{0,4}\right) \oplus\left(\Delta_{-}^{2,2} \otimes \Delta_{-}^{4,0} \otimes \Delta_{+}^{0,4}\right)
$$

of (complexified) Killing spinors.

First of all, let us determine the dimension of the kernel of the nilpotent operators $N^{ \pm}$. To analyse this question we can simply notice that $N^{ \pm}=$ $N_{1}^{ \pm} N_{2}$ where $N_{1}^{ \pm}= \pm \gamma_{2}+\gamma_{3}$ and $N_{2}=\gamma_{1}+\gamma_{4}$ are commuting nilpotent operators. The kernel of $N^{ \pm}$is therefore the subspace generated by the kernels of $N_{1}$ and $N_{2}$, which has $\frac{3}{4}$ the dimension of the space.

Alternatively, the equation $N^{ \pm} \varepsilon=0$ is equivalent to

$$
\left(\gamma_{14}+\gamma_{23}-\gamma_{1234}\right) \varepsilon=\varepsilon
$$

The solution to this eigenvalue problem shows that the action on $\mathrm{AdS}_{3}$ preserves $\frac{3}{4}$ of the supersymmetry.

It is convenient for the full discussion of the supersymmetry preserved by these quotients to determine how the nilpotent operators act in each of the subspaces of (complexified) Killing spinors. We first observe that $N^{ \pm}$ acts trivially on $\Delta_{\mp}^{2,2}$. Indeed, a simple calculation in $\mathrm{C} \ell(2,2)$ shows that

$$
N^{ \pm}\left(\nVdash \mp \gamma_{1234}\right)=0 \text {. }
$$

Since the kernel of $N^{ \pm}$in $\Delta_{+}^{2,2} \oplus \Delta_{-}^{2,2}$ is three-dimensional, we learn that $N^{ \pm}$ has a one-dimensional kernel on $\Delta_{ \pm}^{2,2}$.

To analyse the equation $S \varepsilon=0$, let us observe that $S$ has weights $\pm \frac{1}{2}\left(\theta_{1}-\right.$ $\left.\theta_{2}\right)$ in $\Delta_{+}^{4,0}$ and $\pm \frac{1}{2}\left(\theta_{1}+\theta_{2}\right)$ in $\Delta_{-}^{4,0}$. Therefore there are only zero weights when $\theta_{1}= \pm \theta_{2}$ in $\Delta_{ \pm}^{4,0}$ and none otherwise.

In summary, we see that the quotient generated by $\xi^{+}$preserves $\nu=\frac{1}{8}$ of the original supersymmetries in type IIB when $\theta_{1}=\theta_{2}$. That is, it has four supercharges which live in

$$
\left(\operatorname{ker} N^{+} \cap \Delta_{+}^{2,2}\right) \otimes\left[\Delta_{+}^{4,0} \otimes \Delta_{+}^{0,4}\right] .
$$

Furthermore, it preserves $\nu=\frac{1}{4}$ in type IIB when $\theta_{1}=-\theta_{2}$. These are all eight supercharges living in

$$
\Delta_{-}^{2,2} \otimes\left[\Delta_{-}^{4,0} \otimes \Delta_{+}^{0,4}\right] .
$$

It has no supercharges whenever $\theta_{1} \neq \pm \theta_{2}$. 
On the other hand, the quotient generated by $\xi^{-}$preserves $\nu=\frac{1}{8}$ of the original supersymmetries in type IIB when $\theta_{1}=-\theta_{2}$. That is, it has four supercharges which live in

$$
\left(\operatorname{ker} N^{-} \cap \Delta_{-}^{2,2}\right) \otimes\left[\Delta_{-}^{4,0} \otimes \Delta_{+}^{0,4}\right] .
$$

Furthermore, it preserves $\nu=\frac{1}{4}$ in type IIB when $\theta_{1}=\theta_{2}$. These are all eight supercharges living in

$$
\Delta_{+}^{2,2} \otimes\left[\Delta_{+}^{4,0} \otimes \Delta_{+}^{0,4}\right] .
$$

It has no supercharges whenever $\theta_{1} \neq \pm \theta_{2}$.

In six-dimensional supergravity, $\xi^{ \pm}$break all supersymmetry when $\theta_{1} \neq$ $-\theta_{2}$, whereas if $\theta_{1}=-\theta_{2}, \xi^{+}$preserves all supersymmetry and $\xi^{-}$preserves $\nu=\frac{1}{2}$. As shown in [50] and conjectured in [67], the quotient by $\xi^{+}$is the near-horizon geometry [68] of the critically over-rotating black hole [69, 70].

\subsection{5 $\xi=\xi_{A}^{(8)}+\xi_{S}$}

In this case, the Killing vector field is

$$
\xi^{ \pm}=\mp \boldsymbol{e}_{12}-\boldsymbol{e}_{13} \pm \boldsymbol{e}_{24}+\boldsymbol{e}_{34}+\varphi\left(\boldsymbol{e}_{34} \pm \boldsymbol{e}_{12}\right)+\theta_{1} R_{12}+\theta_{2} R_{34},
$$

where $\theta_{1} \geq\left|\theta_{2}\right|>\varphi>0$, to ensure that $\xi^{ \pm}$is everywhere spacelike. It integrates to the following free action of $\Gamma \cong \mathbb{R}$ on $\mathbb{R}^{2,2}$ :

$$
\left(\begin{array}{l}
x^{1} \\
x^{2} \\
x^{3} \\
x^{4}
\end{array}\right) \mapsto\left(\begin{array}{l}
x^{1} \cos \varphi t \pm x^{2} \sin \varphi t+t\left(\left(x^{1}-x^{4}\right) \sin \varphi t+\left(\mp x^{2}+x^{3}\right) \cos \varphi t\right) \\
x^{2} \cos \varphi t \mp x^{1} \sin \varphi t \pm t\left(\left(x^{1}-x^{4}\right) \cos \varphi t+\left( \pm x^{2}-x^{3}\right) \sin \varphi t\right) \\
x^{3} \cos \varphi t-x^{4} \sin \varphi t+t\left(\left(x^{1}-x^{4}\right) \cos \varphi t+\left( \pm x^{2}-x^{3}\right) \sin \varphi t\right) \\
x^{4} \cos \varphi t+x^{3} \sin \varphi t+t\left(\left(x^{1}-x^{4}\right) \sin \varphi t+\left(\mp x^{2}+x^{3}\right) \cos \varphi t\right)
\end{array}\right)
$$

which lifts to a free action on $\mathrm{AdS}_{3}$. The resulting quotient is smooth and, since $\Gamma \cong \mathbb{R}$, is also spin.

The analysis of supersymmetry follows closely the one in the previous section. We first observe that the action of $\xi$ on Killing spinors is given by the sum of two commuting operators: the same nilpotent operator as in the previous case,

$$
N^{ \pm}=\mp \gamma_{12}-\gamma_{13} \pm \gamma_{24}+\gamma_{34},
$$

and a semisimple one

$$
S^{ \pm}=\varphi\left(\gamma_{34} \pm \gamma_{12}\right)+\theta_{1} \Gamma_{12}+\theta_{2} \Gamma_{34} .
$$


Once again, the invariant Killing spinors correspond to the intersection of the kernels of $N^{ \pm}$and $S^{ \pm}$acting on the space

$$
\left(\Delta_{+}^{2,2} \otimes \Delta_{+}^{4,0} \otimes \Delta_{+}^{0,4}\right) \oplus\left(\Delta_{-}^{2,2} \otimes \Delta_{-}^{4,0} \otimes \Delta_{+}^{0,4}\right)
$$

of (complexified) Killing spinors.

Since we already know the kernel of $N^{ \pm}$from the previous section, we summarise this discussion as follows. To find the amount of supersymmetry preserved by the quotient along $\xi^{ \pm}$, we need to find the invariants of

$$
\theta_{1} \Gamma_{12}+\theta_{2} \Gamma_{34} \quad \text { acting on } \Delta_{ \pm}^{4,0} \otimes \Delta_{+}^{0,4}
$$

and those of

$$
\varphi\left( \pm \gamma_{12}+\gamma_{34}\right)+\theta_{1} \Gamma_{12}+\theta_{2} \Gamma_{34} \quad \text { acting on } \Delta_{\mp}^{2,2} \otimes \Delta_{\mp}^{4,0} \otimes \Delta_{+}^{0,4} .
$$

Let us consider $\xi^{+}$first. The weights of $\theta_{1} \Gamma_{12}+\theta_{2} \Gamma_{34}$ on $\Delta_{+}^{4,0}$ are $\pm \frac{1}{2}\left(\theta_{1}-\right.$ $\left.\theta_{2}\right)$, whereas those of $\varphi\left(\gamma_{12}+\gamma_{34}\right)+\theta_{1} \Gamma_{12}+\theta_{2} \Gamma_{34}$ on $\Delta_{-}^{2,2} \otimes \Delta_{-}^{4,0}$ are $\pm \varphi \pm$ $\frac{1}{2}\left(\theta_{1}+\theta_{2}\right)$. The zero weights occur when $\theta_{1}=\theta_{2}>\varphi>0$ with multiplicity 4 and when $2 \varphi=\theta_{1}+\theta_{2}$ with $\theta_{1}>-\theta_{2}>\varphi>0$, also with multiplicity 4 . In either case there are 4 supersymmetries and hence the quotient preserves a fraction $\nu=\frac{1}{8}$ of the IIB supersymmetry . No further enhancement of supersymmetry is possible given the range of parameters defined by the Weyl group action, the $\mathrm{SO}(2,2)$ action and the causality constraint on $\xi^{+}$. Notice that the first supersymmetric example $\left(\theta_{1}=\theta_{2}>\varphi>0\right)$ provides an example of the phenomenon of turning on a deformation parameter $(\varphi)$ without decreasing the supersymmetry preserved by the quotient.

For the six-dimensional theories, the quotient along $\xi^{+}$is half-BPS whenever $2 \varphi=\theta_{1}+\theta_{2}$ and non-supersymmetric otherwise.

Going on to $\xi^{-}$, the weights of $\theta_{1} \Gamma_{12}+\theta_{2} \Gamma_{34}$ on $\Delta_{-}^{4,0}$ are $\pm \frac{1}{2}\left(\theta_{1}+\theta_{2}\right)$, whereas those of $\varphi\left(-\gamma_{12}+\gamma_{34}\right)+\theta_{1} \Gamma_{12}+\theta_{2} \Gamma_{34}$ on $\Delta_{+}^{2,2} \otimes \Delta_{+}^{4,0}$ are $\pm \varphi \pm \frac{1}{2}\left(\theta_{1}-\right.$ $\left.\theta_{2}\right)$. The zero weights occur when $\theta_{1}=-\theta_{2}>\varphi>0$ with multiplicity 4 , and when $2 \varphi=\theta_{1}-\theta_{2}$ with $\theta_{1}>\theta_{2}>\varphi>0$, also with multiplicity 4 . In either case there are 4 supersymmetries and hence the quotient preserves a fraction $\nu=\frac{1}{8}$ of the IIB supersymmetry. No further supersymmetry enhancement is allowed in this case either. The same comment on the phenomenon of free turning of deformation parameter, supersymmetry wise, applies to $\theta_{1}=-\theta_{2}$.

Again for the six-dimensional theories, the quotient along $\xi^{-}$is half-BPS whenever $\theta_{1}=-\theta_{2}$ and non-supersymmetric otherwise. 
It should be remarked that for the IIB quotients there exists an enhancement of supersymmetry to $\nu=\frac{1}{4}$ by allowing the norm of $\xi$ to vanish at places.

\subsection{6 $\xi=\xi_{A}^{(10)}+\xi_{S}$}

In this case, the Killing vector field is

$$
\xi=\varphi_{1} \boldsymbol{e}_{12}+\varphi_{2} \boldsymbol{e}_{34}+\theta_{1} R_{12}+\theta_{2} R_{34},
$$

where $\varphi_{1} \geq\left|\varphi_{2}\right| \geq 0$, otherwise $\xi_{A}^{(10)}$ would not be bounded from below and $\theta_{1} \geq\left|\theta_{2}\right|>\left|\varphi_{2}\right|$, so that $\xi$ is everywhere spacelike. By rescaling $\xi$ we may further set $\varphi_{1}=1$. The action of $\xi_{A}^{(10)}$ integrates on $\mathbb{R}^{2,2}$ to

$$
\left(\begin{array}{l}
x^{1} \\
x^{2} \\
x^{3} \\
x^{4}
\end{array}\right) \mapsto\left(\begin{array}{c}
x^{1} \cos \varphi_{1} t+x^{2} \sin \varphi_{1} t \\
x^{2} \cos \varphi_{1} t-x^{1} \sin \varphi_{1} t \\
x^{3} \cos \varphi_{2} t-x^{4} \sin \varphi_{2} t \\
x^{4} \cos \varphi_{2} t+x^{3} \sinh \varphi_{2} t
\end{array}\right)
$$

However in the simply-connected $\mathrm{AdS}_{3}$ these orbits are not periodic and hence $\xi$ integrates to an action of $\Gamma \cong \mathbb{R}$. There are clearly no stabilisers and hence the quotient is smooth, and since $\Gamma \cong \mathbb{R}$ it is also spin. (This would be different in the quadric in $\mathbb{R}^{2,2}$, where the analysis of smoothness would be much more delicate.)

Let us move to discuss the supersymmetry of these quotients, noting that $\varphi_{2}$ is allowed to vanish. The weights of $\xi$ in the (complexified) Killing spinor representation (6.2) are given by

$$
\pm \frac{1}{2}\left(\varphi_{1}-\varphi_{2} \pm\left(\theta_{1}-\theta_{2}\right)\right) \quad \pm \frac{1}{2}\left(\varphi_{1}+\varphi_{2} \pm\left(\theta_{1}+\theta_{2}\right)\right)
$$

respectively, with uncorrelated signs and each with multiplicity two. There are two possible conditions for zero weights consistent with our range of parameters, each giving rise to 4 supersymmetries and hence preserving a fraction $\nu=\frac{1}{8}$ of the IIB supersymmetry:

- $\varphi_{1}-\varphi_{2}=\theta_{1}-\theta_{2}$; or

- $\varphi_{1}+\varphi_{2}=\theta_{1}+\theta_{2}$.

Even though these two conditions cannot be satisfied simultaneously and still comply with the conditions on the parameters coming from demanding that the orbits be everywhere spacelike, it is still possible to have enhancement of supersymmetry for the two cases : 
- $\varphi=\varphi_{2}$ and $\theta_{1}=\theta_{2}$; or

- $\varphi_{1}=-\varphi_{2}$ and $\theta_{1}=-\theta_{2}$.

They preserve $\nu=\frac{1}{4}$.

For both of the two six-dimensional theories under consideration, the condition $\varphi_{1}+\varphi_{2}=\theta_{1}+\theta_{2}$ gives rise to half-BPS quotients, whereas $\varphi_{1}=$ $-\varphi_{2}, \theta_{1}=-\theta_{2}$ preserves all the supersymmetry of the vacuum. All other cases listed above break all supersymmetry. As conjectured in [67] and shown recently in [50], the maximally supersymmetric quotient is the near-horizon geometry [68] of the over-rotating black hole [69, 70].

6.1.7 $\xi=\xi_{A}^{(11)}+\xi_{S}$

We let $\beta_{1}= \pm \beta_{2}=\beta$ in case (11) to arrive at the Killing vector

$$
\xi^{ \pm}=\beta\left(\boldsymbol{e}_{13} \pm \boldsymbol{e}_{24}\right)+\theta_{1} R_{12}+\theta_{2} R_{34},
$$

where now the $\theta_{i}$ are allowed to be zero provided that $\beta$ is not. Since we already discussed the case $\beta=0$, we will now concentrate on $\beta \neq 0$, whence we can rescale $\xi^{ \pm}$such that $\beta=1$. The anti-de Sitter component integrates to the following action on $\mathbb{R}^{2,2}$ :

$$
\left(\begin{array}{l}
x^{1} \\
x^{2} \\
x^{3} \\
x^{4}
\end{array}\right) \mapsto\left(\begin{array}{l}
x^{1} \cosh t-x^{3} \sinh t \\
x^{2} \cosh t \mp x^{4} \sinh t \\
x^{3} \cosh t-x^{1} \sinh t \\
x^{4} \cosh t \mp x^{2} \sinh t
\end{array}\right)
$$

which is clearly a free action of $\Gamma \cong \mathbb{R}$. This also lifts to a free action of $\Gamma$ on $\mathrm{AdS}_{3}$ and hence on the background for all values of $\theta_{i}$. The resulting quotient is therefore smooth and, since $\Gamma \cong \mathbb{R}$, also spin.

The Killing vector defines an action on the (complexified) Killing spinor representation which decomposes into two commuting semisimple pieces:

$$
S_{1}^{ \pm}=\gamma_{13} \pm \gamma_{24}
$$

and

$$
S_{2}=\theta_{1} \Gamma_{12}+\theta_{2} \Gamma_{34} .
$$

On a complex vector space, they are both diagonalisable, but whereas $S_{1}$ has real eigenvalues, those of $S_{2}$ are imaginary. Therefore the supersymmetries of the quotient consist of Killing spinors in the kernel of both. 
We observe that $S_{1}^{ \pm} \varepsilon=0$ if and only if $\varepsilon \in \Delta_{ \pm}^{2,2} \otimes\left[\Delta_{ \pm}^{4,0} \otimes \Delta_{+}^{0,4}\right]$. This means that $S_{2} \varepsilon=0$ if and only if $\theta_{1}= \pm \theta_{2}$. Hence either quotient preserves a fraction $\nu=\frac{1}{4}$ of the IIB supersymmetry. This is still the case if $\theta_{i}=0$, which corresponds to the selfdual orbifolds introduced in [39] and studied recently in [40]. Thus, these are examples of quotients in which by turning on the $\theta_{i}$ in an appropriate way no supersymmetry is lost. Note that this phenomenon originates in the way the Killing vector acts on the Killing spinors and on the space in which the latter live. Therefore this phenomenon depends on dimension and will not be a feature of all $\mathrm{AdS}_{p+1}$ quotients.

Finally we observe that whereas $\xi^{+}$preserves no supersymmetry in the six-dimensional theories, $\xi^{-}$preserves all. Indeed, the quotient by $\xi^{-}$is the near-horizon geometry [68] of the rotating black hole of $[69,70]$.

\subsubsection{Summary}

The smooth spacelike supersymmetric quotients of $\mathrm{AdS}_{3} \times S^{3}$ are summarised in Table 6, where we have also indicated the fraction of supersymmetry preserved by each of the theories under consideration: type IIB and sixdimensional $(1,0)$ and $(2,0)$ supergravities. The $(2,0)$ fraction is the same as the $(1,0)$ fraction and is hence not written explicitly.

\subsection{Supersymmetric quotients of $\mathrm{AdS}_{4} \times S^{7}$}

The $\mathrm{AdS}_{4} \times S^{7}$ vacuum of eleven-dimensional supergravity is such that $\mathrm{AdS}_{4}$ has radius of curvature $R_{A}=R$ and $S^{7}$ has radius of curvature $R_{S}=2 R$. Letting $s>0$ denote the scalar curvature of the sphere, the 4 -form is given by

$$
F=\sqrt{\frac{6}{7} s} \operatorname{dvol}_{A}
$$

where $\mathrm{dvol}_{A}$ is the volume form of $\mathrm{AdS}_{4}$. This vacuum solution has thirtytwo supersymmetries. In this section we will study the smooth supersymmetric quotients.

The situation here is analogous to the one in the previous section. The spherical component $\xi_{S}$ of the Killing vector is given by

$$
\xi_{S}=\theta_{1} R_{12}+\theta_{2} R_{34}+\theta_{3} R_{56}+\theta_{4} R_{78}
$$

whose norm is bounded above and below by

$$
m^{2} R_{S}^{2} \leq\left|\xi_{S}\right|^{2} \leq M^{2} R_{S}^{2}
$$




\begin{tabular}{|c|c|c|c|c|}
\hline \multirow[b]{2}{*}{ Killing vector } & \multicolumn{2}{|c|}{ Conditions for } & \multicolumn{2}{|c|}{$\nu$ in } \\
\hline & Causality/Weyl & Supersymmetry & IIB & $(1,0)$ \\
\hline$\xi_{S}=\theta_{1} R_{12}+\theta_{2} R_{34}$ & $\theta_{1} \geq\left|\theta_{2}\right|>0$ & $\begin{array}{l}\theta_{i}=1 \\
\theta_{i}=(-1)^{i}\end{array}$ & $\begin{array}{l}\frac{1}{4} \\
\frac{1}{4}\end{array}$ & $\begin{array}{l}0 \\
1\end{array}$ \\
\hline$\varphi \boldsymbol{e}_{34}+\xi_{S}$ & $\begin{array}{l}\varphi>0 \\
\theta_{1} \geq\left|\theta_{2}\right|>0\end{array}$ & $\begin{array}{l}\varphi=2, \theta_{i}=1 \\
\varphi=2, \theta_{i}=(-1)^{i}\end{array}$ & $\begin{array}{l}\frac{1}{8} \\
\frac{1}{8} \\
\end{array}$ & $\begin{array}{l}\frac{1}{4} \\
0\end{array}$ \\
\hline $\begin{aligned}-\boldsymbol{e}_{12}- & \boldsymbol{e}_{13} \\
& +\boldsymbol{e}_{24}+\boldsymbol{e}_{34}+\xi_{S}\end{aligned}$ & $\theta_{1} \geq\left|\theta_{2}\right|>0$ & $\begin{array}{l}\theta_{1}=\theta_{2} \\
\theta_{1}=-\theta_{2}\end{array}$ & $\begin{array}{l}\frac{1}{8} \\
\frac{1}{4}\end{array}$ & $\begin{array}{l}0 \\
1\end{array}$ \\
\hline $\begin{array}{l}\boldsymbol{e}_{12}-\boldsymbol{e}_{13} \\
\quad-\boldsymbol{e}_{24}+\boldsymbol{e}_{34}+\xi_{S}\end{array}$ & $\theta_{1} \geq\left|\theta_{2}\right|>0$ & $\begin{array}{l}\theta_{1}=\theta_{2} \\
\theta_{1}=-\theta_{2}\end{array}$ & $\begin{array}{l}\frac{1}{4} \\
\frac{1}{8}\end{array}$ & $\begin{array}{l}0 \\
\frac{1}{2}\end{array}$ \\
\hline $\begin{array}{r}-\boldsymbol{e}_{12}-\boldsymbol{e}_{13}+\boldsymbol{e}_{24}+\boldsymbol{e}_{34} \\
+\varphi\left(\boldsymbol{e}_{34}+\boldsymbol{e}_{12}\right)+\xi_{S} \\
\end{array}$ & $\theta_{1} \geq\left|\theta_{2}\right|>\varphi>0$ & $\begin{array}{l}\theta_{1}=\theta_{2} \\
\varphi=\frac{1}{2}\left(\theta_{1}+\theta_{2}\right)\end{array}$ & $\begin{array}{l}\frac{1}{8} \\
\frac{1}{8}\end{array}$ & $\begin{array}{l}0 \\
\frac{1}{2} \\
\end{array}$ \\
\hline $\begin{array}{l}\boldsymbol{e}_{12}-\boldsymbol{e}_{13}-\boldsymbol{e}_{24}+\boldsymbol{e}_{34} \\
+\varphi\left(\boldsymbol{e}_{34}-\boldsymbol{e}_{12}\right)+\xi_{S}\end{array}$ & $\theta_{1} \geq\left|\theta_{2}\right|>\varphi>0$ & $\begin{array}{l}\theta_{1}=-\theta_{2} \\
\varphi=\frac{1}{2}\left(\theta_{1}-\theta_{2}\right)\end{array}$ & $\begin{array}{l}\frac{1}{8} \\
\frac{1}{8} \\
\end{array}$ & $\begin{array}{l}\frac{1}{2} \\
0\end{array}$ \\
\hline $\boldsymbol{e}_{12}+\varphi \boldsymbol{e}_{34}+\xi_{S}$ & $\begin{array}{l}1 \geq|\varphi| \\
\theta_{1} \geq\left|\theta_{2}\right|>|\varphi|\end{array}$ & $\begin{array}{l}1-\varphi=\theta_{1}-\theta_{2} \\
1+\varphi=\theta_{1}+\theta_{2} \\
\varphi=1, \theta_{1}=\theta_{2} \\
\varphi=-1, \theta_{1}=-\theta_{2}\end{array}$ & $\begin{array}{l}\frac{1}{8} \\
\frac{1}{8} \\
\frac{1}{4} \\
\frac{1}{4} \\
\end{array}$ & $\begin{array}{l}0 \\
\frac{1}{2} \\
0 \\
1\end{array}$ \\
\hline $\boldsymbol{e}_{13}+\boldsymbol{e}_{24}+\xi_{S}$ & $\theta_{1} \geq\left|\theta_{2}\right| \geq 0$ & $\theta_{1}=\theta_{2}$ & $\frac{1}{4}$ & 0 \\
\hline $\boldsymbol{e}_{13}-\boldsymbol{e}_{24}+\xi_{S}$ & $\theta_{1} \geq\left|\theta_{2}\right| \geq 0$ & $\theta_{1}=-\theta_{2}$ & $\frac{1}{4}$ & 1 \\
\hline
\end{tabular}

Table 6: Smooth spacelike supersymmetric quotients of $\mathrm{AdS}_{3} \times S^{3}$

where

$$
m^{2}=\min _{i} \theta_{i}^{2} \quad \text { and } \quad M^{2}=\max _{i} \theta_{i}^{2} .
$$

It is easy to see that these bounds are sharp. The Weyl group acts by permuting the $\theta_{i}$ and changing an even number of their signs (see, e.g., [71, Section 12.1]). This means that we can order them so that $\theta_{1} \geq \theta_{2} \geq \theta_{3} \geq$ 
$\left|\theta_{4}\right|$. Clearly $m^{2}$ and $M^{2}$ are Weyl-invariant.

Provided that $m^{2}>0$, we will be able to use Killing vectors in $\mathrm{AdS}_{4}$ which are not necessarily everywhere spacelike, provided their norms are bounded below. From the results in Section 4.2, the only Killing vectors $\xi_{A}$ on $\mathrm{AdS}_{4}$ which satisfy this condition are the ones labelled (4), (5), (6) and (11) with $\beta_{1}=\beta_{2}$. The resulting Killing vectors are everywhere spacelike with norms bounded below by $m^{2} R_{S}^{2}$ for cases (4), (5) and (6); and by $m^{2} R_{S}^{2}+\beta_{1}^{2} R_{A}^{2}$ for case (11).

\subsection{1 $\xi=\xi_{S}$}

For $\xi$ to be everywhere nonvanishing (and hence spacelike) we require all $\theta_{i}$ to be nonzero. This guarantees that the action of $\Gamma$ is locally free. To analyse the stabilisers it is convenient to think of $S^{7}$ an embedded in $\mathbb{C}^{4}$ with standard coordinates $z_{i}, i=1,2,3,4$ as the quadric

$$
\left|z_{1}\right|^{2}+\left|z_{2}\right|^{2}+\left|z_{3}\right|^{2}+\left|z_{4}\right|^{2}=4 R^{2}
$$

The action of $\xi$ integrates to

$$
\left(z_{1}, z_{2}, z_{3}, z_{4}\right) \mapsto\left(e^{i \theta_{1} t} z_{1}, e^{i \theta_{2} t} z_{2}, e^{i \theta_{3} t} z_{3}, e^{i \theta_{4} t} z_{4}\right) .
$$

The quotient will not be Hausdorff unless the integral curves are periodic (compare with the discussion in [25, Section 3.2]), whence there is a smallest positive number $T$ for which

$$
\left(e^{i \theta_{1} T} z_{1}, e^{i \theta_{2} T} z_{2}, e^{i \theta_{3} T} z_{3}, e^{i \theta_{4} T} z_{4}\right)=\left(z_{1}, z_{2}, z_{3}, z_{4}\right),
$$

for all $\left(z_{1}, z_{2}, z_{3}, z_{4}\right)$. This implies that there are integers $p_{i}$ such that $\theta_{i} T=$ $2 \pi p_{i}$ and since $T$ is the smallest such positive integer, the $p_{i}$ are coprime. Using the freedom to rescale the Killing vector, we can take

$$
\xi=p_{1} R_{12}+p_{2} R_{34}+p_{3} R_{56}+p_{4} R_{78} \quad \text { with } \operatorname{gcd}\left(p_{1}, p_{2}, p_{3}, p_{4}\right)=1,
$$

which integrates to a circle action

$$
\left(z_{1}, z_{2}, z_{3}, z_{4}\right) \mapsto\left(e^{i p_{1} t} z_{1}, e^{i p_{2} t} z_{2}, e^{i p_{3} t} z_{3}, e^{i p_{4} t} z_{4}\right)
$$

with period $2 \pi$. The action has trivial stabilisers if for all $\left(z_{1}, z_{2}, z_{3}, z_{4}\right)$ obeying (6.4), the equation

$$
\left(z_{1}, z_{2}, z_{3}, z_{4}\right)=\left(e^{i p_{1} t} z_{1}, e^{i p_{2} t} z_{2}, e^{i p_{3} t} z_{3}, e^{i p_{4} t} z_{4}\right)
$$

holds only for $t \in 2 \pi \mathbb{Z}$. By looking at the points $(2 R, 0,0,0),(0,2 R, 0,0)$, etc we see that $p_{i}$ have to be \pm 1 giving $2^{4}=16$ possibilities which, taking 
into account the action of the Weyl group, are reduced to only two: $p_{1}=$ $p_{2}=p_{3}=1$ and $p_{4}= \pm 1$. For $p_{4}=1$, the resulting quotient is $\mathbb{C P}^{3}$, which as we saw above, admits a spin structure. For $p_{4}=-1$, we also obtain $\mathbb{C P}^{3}$ but with the opposite orientation. Indeed, notice that the two cases are related by an orientation-reversing isometry in $\mathrm{O}(8)$. In either case we have a quotient with a spin structure. We shall now discuss in which cases $\mathrm{AdS}_{4} \times \mathbb{C P}^{3}$ backgrounds do preserve some amount of supersymmetry $[60,61]$.

Let

$$
\xi^{ \pm}=R_{12}+R_{34}+R_{56} \pm R_{78}
$$

generate a one-parameter subgroup $\Gamma^{ \pm} \subset \operatorname{Spin}(8)$. The Killing spinors in $S^{7}$ are in the representation $\Delta_{-}^{8,0}$ of $\operatorname{Spin}(8)$. The weights of $\xi^{+}$on $\Delta_{-}^{8,0}$ are \pm 1 with multiplicity 4 , whereas those of $\xi^{-}$are $(0, \pm 2)$ with multiplicities 6 and 1 , respectively. Therefore quotienting by $\Gamma^{+}$breaks all supersymmetry, whereas quotienting by $\Gamma^{-}$preserves a fraction $\nu=\frac{3}{4}$.

Alternatively, we can solve (6.1) explicitly and arrive at the same conclusion. Given the one-to-one correspondence between Killing spinors on $S^{7}$ and negative chirality spinors on $\mathbb{R}^{8}$, we can manifestly evaluate (6.1) for the particular case of $\xi=\xi_{S}$ being considered now. This gives rise to

$$
\left(\Gamma_{12}+\Gamma_{34}+\Gamma_{56} \pm \Gamma_{78}\right) \varepsilon=0 .
$$

Since $\Gamma_{12}$ in invertible, the above equation is equivalent to

$$
\left(\Gamma_{1234}+\Gamma_{1256} \pm \Gamma_{1278}\right) \varepsilon=\varepsilon,
$$

where the square of all antisymmetric combinations of gamma matrices equals one. The solution to this kind of eigenvalue problem was described in detail in [72]. There is a subtlety though, which is worth mentioning. The solution $\varepsilon$ to equation (6.5) has to be found in the subspace of negative chirality spinors. In this subspace, the three operators appearing in the left hand side of equation (6.5) are no longer independent. In particular, the third one can be written as a product of the first two ones, that is, $\Gamma_{1278}=\Gamma_{1234} \Gamma_{1256}$. Under these circumstances, as pointed out in [73] and generalised in [72], preservation of an exotic amount of supersymmetry is allowed. An explicit matrix realisation for these operators

$$
\begin{aligned}
& \Gamma_{1234}=\operatorname{diag}(1,1,-1,-1) \otimes \nVdash \\
& \Gamma_{1256}=\operatorname{diag}(1,-1,1,-1) \otimes \nVdash \\
& \Gamma_{1278}=\operatorname{diag}(1,-1,-1,1) \otimes \nVdash,
\end{aligned}
$$

defines an eigenvalue problem given by

$$
\operatorname{diag}\left(M-\lambda_{1}^{ \pm}, M-\lambda_{2}^{ \pm}, M-\lambda_{3}^{ \pm}, M-\lambda_{4}^{ \pm}\right) \otimes \nVdash \varepsilon=0,
$$


where $M=1$, as can be seen from (6.5), and $\lambda_{i}^{ \pm}$are the diagonal elements of the matrix appearing in the left hand side of equation (6.5) in the realisation given above. It is straightforward to check that for $p_{4}=1$, non of the diagonal elements of the above eigenvalue problem vanish. Thus, the corresponding spinor $\varepsilon$ vanishes. Therefore, $\mathbb{C P}^{3}$ breaks all the supersymmetry. On the other hand, when $p_{4}=-1, \lambda_{i}^{-}=1 i=1,2,3$. Thus, there exists a non-trivial spinor solving the eigenvalue problem. The amount of supersymmetry preserved is, indeed, given by $\nu=3 / 4$, since three out of four of the eigenvalues vanish.

We now turn our attention to the four possible families of reductions where the anti-de Sitter component of the Killing vector is nonzero.

\subsection{2 $\xi=\xi_{A}^{(4)}+\xi_{S}$}

This case is very similar to the previous one, with the possibility of adding a spatial rotation $\xi_{A}^{(4)}=\varphi \boldsymbol{e}_{34}$ to $\xi$. The same analysis as before shows that $\varphi T=2 \pi n$ for some integer $n$ which is coprime to the $p_{i}$, whence after rescaling the Killing vector it becomes

$\xi=n \boldsymbol{e}_{34}+p_{1} R_{12}+p_{2} R_{34}+p_{3} R_{56}+p_{4} R_{78} \quad$ with $\operatorname{gcd}\left(n, p_{1}, p_{2}, p_{3}, p_{4}\right)=1$.

As before, the triviality of the stabilisers demands that $p_{i}= \pm 1$, and hence up to rescaling $\xi$ and conjugating by the Weyl group of $\mathrm{SO}(8)$ one has

$$
\xi=n \boldsymbol{e}_{34}+R_{12}+R_{34}+R_{56} \pm R_{78} \quad \text { with } n=1,2, \ldots
$$

Let us employ the convention that $\gamma_{i}$ denote the $\gamma$-matrices for $\mathrm{C} \ell(2,3)$ and $\Gamma_{a}$ denote those of $\mathrm{C} \ell(8)$. Then the element in $\mathfrak{s o}(2,3) \oplus \mathfrak{s o}(8) \subset \mathrm{C} \ell(2,11)$ corresponding to $\xi$ is given by

$$
X=n \gamma_{34}+\Gamma_{12}+\Gamma_{34}+\Gamma_{56} \pm \Gamma_{78} .
$$

Exponentiating this element we get

$$
\begin{aligned}
& \widehat{g}(t)=\exp \frac{t X}{2}=\left(\nVdash \cos \frac{n t}{2}+\gamma_{34} \sin \frac{n t}{2}\right)\left(\nVdash \cos \frac{t}{2}+\Gamma_{12} \sin \frac{t}{2}\right) \\
& \times\left(\nVdash \cos \frac{t}{2}+\Gamma_{34} \sin \frac{t}{2}\right)\left(\nVdash \cos \frac{t}{2}+\Gamma_{56} \sin \frac{t}{2}\right)\left(\nVdash \cos \frac{t}{2} \pm \Gamma_{78} \sin \frac{t}{2}\right)
\end{aligned}
$$

and this can only possibly equal $-\nVdash$ at $t=2 \pi$, where

$$
\widehat{g}(2 \pi)=(-1)^{n} \nVdash ;
$$


whence only for $n$ even do we have a spin structure in the quotient.

The analysis of supersymmetry is analogous to the ones given in the previous subsection. The weights of the element

$$
n \gamma_{34}+\Gamma_{12}+\Gamma_{34}+\Gamma_{56}+\Gamma_{78}
$$

on the representation carried by the Killing spinors are $\pm \frac{n}{2} \pm 1$ with uncorrelated signs and multiplicity 8 . There are 16 zero weights when $n=2$ and none otherwise. The resulting supersymmetric quotient is therefore halfBPS. The element

$$
n \gamma_{34}+\Gamma_{12}+\Gamma_{34}+\Gamma_{56}-\Gamma_{78}
$$

has weights $\pm \frac{n}{2}$ with multiplicity 12 and $\pm \frac{n}{2} \pm 2$ with uncorrelated signs and multiplicity 2 . Since $n \neq 0$, there are 4 zero weights when $n=4$ and none otherwise. The resulting supersymmetric quotient preserves a fraction $\nu=\frac{1}{8}$ of the supersymmetry of the original background.

6.2.3 $\xi=\xi_{A}^{(5)}+\xi_{S}$

Here the Killing vector field is

$$
\xi=e_{13}-e_{34}+\theta_{1} R_{12}+\theta_{2} R_{34}+\theta_{3} R_{56}+\theta_{4} R_{78}
$$

This vector fields integrates to the action of $\Gamma \cong \mathbb{R}$. The orbits are generically non-periodic, but on the hyperbola in $\mathrm{AdS}_{4}$ consisting of points $x_{1}=x_{3}=$ $x_{4}=0$ and $x_{2}^{2}=R^{2}+x_{5}^{2}$, the orbits are either not periodic, in which case they would be dense in a submanifold of dimension at least 2 and hence the quotient would not be Hausdorff, or else they are periodic in which case their points have nontrivial stabiliser, namely the subgroup $\Gamma_{0} \subset \Gamma$ consisting of the periods. In either case the quotient is not smooth.

6.2.4 $\xi=\xi_{A}^{(6)}+\xi_{S}$

In this case the Killing vector is

$$
\xi=-\boldsymbol{e}_{12}-\boldsymbol{e}_{13}+\boldsymbol{e}_{24}+\boldsymbol{e}_{34}+\theta_{1} R_{12}+\theta_{2} R_{34}+\theta_{3} R_{56}+\theta_{4} R_{78},
$$

where the $\theta_{i}$ are nonzero for $\xi$ to be everywhere spacelike. Notice that the anti-de Sitter component is never zero, although its norm vanishes. It 
integrates to the following action on $\mathbb{R}^{2,3}$ :

$$
\left(\begin{array}{c}
x^{1} \\
x^{2} \\
x^{3} \\
x^{4} \\
x^{5}
\end{array}\right) \mapsto\left(\begin{array}{c}
x^{1}+t\left(x^{3}-x^{2}\right) \\
x^{2}+t\left(x^{1}-x^{4}\right) \\
x^{3}+t\left(x^{1}-x^{4}\right) \\
x^{4}+t\left(x^{3}-x^{2}\right) \\
x^{5}
\end{array}\right)
$$

which are straight lines ruling the quadric in $\mathbb{R}^{2,3}$ which is locally isometric to $\mathrm{AdS}_{4}$. The combined Killing vector $\xi$ therefore integrates to an action of $\Gamma \cong \mathbb{R}$. It is easy to see that it has trivial stabilisers, since it does on $\mathrm{AdS}_{4}$. Therefore the resulting background $\left(\mathrm{AdS}_{4} \times S^{7}\right) / \Gamma$ is smooth and, since $\Gamma \cong \mathbb{R}$, also spin.

The action of $\xi$ on the representation carried by the Killing spinors breaks up into a sum of commuting nilpotent and semisimple pieces: $N+S$. The $\Gamma$-invariant Killing spinors are precisely those which are annihilated by both $N$ and $S$.

The nilpotent piece

$$
N=\gamma_{13}-\gamma_{34}+\gamma_{12}-\gamma_{24}
$$

in turn breaks up as a product of two anticommuting nilpotent operators $N=N_{1} N_{2}$, where

$$
N_{1}=\gamma_{2}+\gamma_{3} \quad \text { and } \quad N_{2}=\gamma_{1}+\gamma_{4}
$$

$N$ acts on $\Delta^{2,3}$ and its kernel is the subspace generated by the kernels of $N_{1}$ and $N_{2}$. It is three-dimensional.

The semisimple piece is given by

$$
S=\theta_{1} \Gamma_{12}+\theta_{2} \Gamma_{34}+\theta_{3} \Gamma_{56}+\theta_{4} \Gamma_{78}
$$

and its action on $\Delta_{-}^{8,0}$ is determined by the weights of that representation: $\pm \theta_{1} \pm \theta_{2} \pm \theta_{3} \pm \theta_{4}$ with uncorrelated signs whose product is negative and with multiplicity one. The existence of zero weights define hyperplanes in the space of the $\theta_{i}$. Taking into account the ordering of the $\theta_{i}$ and the fact $\theta_{1,2,3}$ are positive, we find that there are three possible hyperplanes:

- $\theta_{1}-\theta_{2}+\theta_{3}+\theta_{4}=0$

- $\theta_{1}+\theta_{2}-\theta_{3}+\theta_{4}=0$; and

- $\theta_{1}-\theta_{2}-\theta_{3}-\theta_{4}=0$. 
Whenever the $\theta_{i}$ belong to one (and only one) of these hyperplanes, there is a two-dimensional subspace of $\Delta_{-}^{8,0}$ which is annihilated by $S$. The resulting quotient therefore has six supersymmetries, and hence preserves a fraction $\nu=\frac{3}{16}$ of the supersymmetry of the eleven-dimensional vacuum. When the $\theta_{i}$ belong to the intersection of the first and third hyperplanes, which happens when $\theta_{1}=\theta_{2}$ and $\theta_{3}=-\theta_{4}$, the kernel of $S$ is four-dimensional. The resulting quotient has 12 supersymmetries, and hence preserves a fraction $\nu=\frac{3}{8}$ of the supersymmetry of the eleven-dimensional vacuum. Finally when $\theta_{i}$ lies in the intersection of all three hyperplanes, which happens when $\theta_{1}=\theta_{2}=\theta_{3}=-\theta_{4}$, the kernel of $S$ is six-dimensional and the resulting quotient has 18 supersymmetries, corresponding to a fraction $\nu=\frac{9}{16}$.

\subsection{5 $\xi=\xi_{A}^{(11)}+\xi_{S}$}

We let $\beta_{1}=\beta_{2}=\beta$ in case (11) to arrive at the Killing vector

$$
\xi=\beta\left(\boldsymbol{e}_{13}+\boldsymbol{e}_{24}\right)+\theta_{1} R_{12}+\theta_{2} R_{34}+\theta_{3} R_{56}+\theta_{4} R_{78},
$$

where now the $\theta_{i}$ are allowed to be zero provided that $\beta$ is not. Since we already discussed the case $\beta=0$, we will now concentrate on $\beta \neq 0$, whence we can rescale $\xi$ such that $\beta=1$. The anti-de Sitter component integrates to the following action on $\mathbb{R}^{2,3}$ :

$$
\left(\begin{array}{c}
x^{1} \\
x^{2} \\
x^{3} \\
x^{4} \\
x^{5}
\end{array}\right) \mapsto\left(\begin{array}{c}
x^{1} \cosh t-x^{3} \sinh t \\
x^{2} \cosh t-x^{4} \sinh t \\
x^{3} \cosh t-x^{1} \sinh t \\
x^{4} \cosh t-x^{2} \sinh t \\
x^{5}
\end{array}\right)
$$

which is clearly a free action of $\Gamma \cong \mathbb{R}$ on the quadric and hence already on the simply-connected $\mathrm{AdS}_{4}$ for all values of $\theta_{i}$. The resulting background $\left(\mathrm{AdS}_{4} \times S^{7}\right) / \Gamma$ is therefore smooth and, since $\Gamma \cong \mathbb{R}$, also spin.

The action of $\xi$ on the Killing spinors breaks up into two commuting semisimple pieces $S_{1}+S_{2}$, where

$$
S_{1}=\gamma_{13}+\gamma_{24}
$$

and

$$
S_{2}=\theta_{1} \Gamma_{12}+\theta_{2} \Gamma_{34}+\theta_{3} \Gamma_{56}+\theta_{4} \Gamma_{78}
$$

is just as before, except that now the $\theta_{i}$ are allowed to vanish. If we complexify the Killing spinors we may diagonalise $S_{1}$ and $S_{2}$ : with $S_{1}$ having real 
eigenvalues and $S_{2}$ imaginary eigenvalues. Therefore a spinor is annihilated by $S_{1}+S_{2}$ if and only if it is annihilated by $S_{1}$ and $S_{2}$ separately.

The kernel of $S_{1}$ on $\Delta^{2,3}$ is clearly two-dimensional, since it corresponds to those spinors $\varepsilon$ obeying $\gamma_{1234} \varepsilon=\varepsilon$.

The analysis of $S_{2}$ is similar to that in the previous section except for the possibility that one or more of the $\theta_{i}$ may vanish. We have the same cases we had before, but where the fractions of supersymmetry are now two thirds of the previous fractions, namely $\nu=\frac{1}{8}, \nu=\frac{1}{4}$ and $\nu=\frac{3}{8}$. In addition we have the case in which all $\theta_{i}$ vanish, which corresponds to a half-BPS quotient.

\subsubsection{Summary}

The smooth spacelike supersymmetric quotients of $\mathrm{AdS}_{4} \times S^{7}$ are summarised in Table 7, where we have also indicated the fraction $\nu$ of the eleven-dimensional supersymmetry preserved by the quotient.

\subsection{Supersymmetric quotients of $\mathrm{AdS}_{5} \times S^{5}$}

In the $\mathrm{AdS}_{5} \times S^{5}$ vacuum of ten-dimensional type IIB supergravity, both spaces have equal radii of curvature $R$. Letting $s>0$ be the scalar curvature of the sphere, the selfdual 5 -form is given by

$$
F=\sqrt{\frac{1}{80} s}\left(\operatorname{dvol}_{A}+\operatorname{dvol}_{S}\right)
$$

where $\operatorname{dvol}_{A}$ and $\mathrm{dvol}_{S}$ are the volume forms of $\mathrm{AdS}_{5}$ and $S^{5}$, respectively. (The bizarre-looking factor of 80 is a consequence of our chosen value for the (constant) dilaton.) This vacuum solution has thirty-two supersymmetries. In this section we will study the smooth supersymmetric quotients.

The situation here is very close to the one in the previous sections. Since the sphere is odd-dimensional, it also admits Killing vectors which are bounded below by a positive number and therefore we will be able to admit Killing vectors in $\mathrm{AdS}_{5}$ which are not necessarily everywhere spacelike, provided their norms are bounded below. From the results in Section 4.3, the only Killing vectors $\xi_{A}$ on $\mathrm{AdS}_{5}$ which satisfy this condition are those labelled (4), (5), (6), (11) for $\beta_{1}=\beta_{2},(16)$, (17), (20) for $\left|\varphi_{2}\right| \geq \varphi_{1}>0$, (21), (23), (24) for $\varphi_{2} \geq \varphi_{3} \geq\left|\varphi_{1}\right|>0$, and (25) for $\beta_{1}=\beta_{2}$. We now analyse each case in more detail. 


\begin{tabular}{|c|l|l|c|}
\hline \multirow{2}{*}{ Killing vector } & \multicolumn{2}{|c|}{ Conditions for } & \\
\hline \hline$\xi_{S}=\theta_{1} R_{12}+\theta_{2} R_{34}$ & \multicolumn{1}{|c|}{ Causality/Weyl } & \multicolumn{1}{|c|}{ Supersymmetry } & $\nu$ \\
$+\theta_{3} R_{56}+\theta_{4} R_{78}$ & $\theta_{1} \geq \theta_{2} \geq \theta_{3} \geq\left|\theta_{4}\right|>0$ & $\theta_{1,2,3}=1, \theta_{4}=-1$ & \\
\hline $\boldsymbol{e}_{34}+\xi_{S}$ & $\varphi \geq 0$ & $\varphi=2, \theta_{i}=1$ & $\frac{3}{4}$ \\
\hline $\boldsymbol{e}_{12}-\boldsymbol{e}_{13}$ & $\theta_{1} \geq \theta_{2} \geq \theta_{3} \geq\left|\theta_{4}\right|>0$ & $\varphi=4, \theta_{1,2,3}=1, \theta_{4}=-1$ & $\frac{1}{2}$ \\
$+\boldsymbol{e}_{24}+\boldsymbol{e}_{34}+\xi_{S}$ & $\theta_{1} \geq \theta_{2} \geq \theta_{3} \geq\left|\theta_{4}\right|>0$ & $\theta_{1} \mp \theta_{2} \pm \theta_{3}=-\theta_{4}$ & $\frac{3}{16}$ \\
& & $\theta_{1}-\theta_{2}-\theta_{3}=\theta_{4}$ & $\frac{3}{16}$ \\
& & $\theta_{1}=\theta_{2}, \theta_{3}=-\theta_{4}$ & $\frac{3}{8}$ \\
& $\theta_{1} \geq \theta_{2} \geq \theta_{3} \geq\left|\theta_{4}\right| \geq 0$ & $\theta_{1} \mp \theta_{2} \pm \theta_{3}=-\theta_{4}$ & $\frac{9}{16}$ \\
\hline $\boldsymbol{e}_{13}+\boldsymbol{e}_{24}+\xi_{S}$ & & $\theta_{1}-\theta_{2}-\theta_{3}=\theta_{4}$ & $\frac{1}{8}$ \\
& & $\theta_{1}=\theta_{2}, \theta_{3}=-\theta_{4}$ & $\frac{1}{8}$ \\
& & $\theta_{1}=\theta_{2}=\theta_{3}=-\theta_{4}$ & $\frac{1}{4}$ \\
& & $\theta_{i}=0$ & $\frac{3}{8}$ \\
& & & $\frac{1}{2}$ \\
\hline
\end{tabular}

Table 7: Smooth spacelike supersymmetric quotients of $\mathrm{AdS}_{4} \times S^{7}$

Throughout this section the spherical component $\xi_{S}$ of the Killing vector is given by

$$
\xi_{S}=\theta_{1} R_{12}+\theta_{2} R_{34}+\theta_{3} R_{56},
$$

whose norm obeys $\left|\xi_{S}\right|^{2} \geq \min _{i} \theta_{i}^{2} R^{2}$, where $R$ is the radius of curvature of both $S^{5}$ and $\mathrm{AdS}_{5}$. The action of the Weyl group allows us to order them as $\theta_{1} \geq \theta_{2} \geq\left|\theta_{3}\right|$.

\subsection{1 $\xi=\xi_{S}$}

This case is very similar to the analogous reduction of $\mathrm{AdS}_{4} \times S^{7}$, so we will be brief. The action is locally free provided all $\theta_{i}$ are nonzero. To analyse the stabilisers it is convenient to think of $S^{5}$ as embedded in $\mathbb{C}^{3}$ with standard 
coordinates $z_{i}, i=1,2,3$, as the quadric

$$
\left|z_{1}\right|^{2}+\left|z_{2}\right|^{2}+\left|z_{3}\right|^{2}=R^{2}
$$

The action of $\xi$ integrates to

$$
\left(z_{1}, z_{2}, z_{3}\right) \mapsto\left(e^{i \theta_{1} t} z_{1}, e^{i \theta_{2} t} z_{2}, e^{i \theta_{3} t} z_{3}\right),
$$

where we demand periodicity for the quotient to be Hausdorff. Let $T$ be the period, so that there are integers $p_{i}$ such that $\theta_{i} T=2 \pi p_{i}$ and since $T$ is the smallest such positive integer, the $p_{i}$ are coprime. Using the freedom to rescale the Killing vector, we can take

$$
\xi=p_{1} R_{12}+p_{2} R_{34}+p_{3} R_{56} \quad \text { with } \operatorname{gcd}\left(p_{1}, p_{2}, p_{3}\right)=1,
$$

which integrates to a circle action

$$
\left(z_{1}, z_{2}, z_{3}\right) \mapsto\left(e^{i p_{1} t} z_{1}, e^{i p_{2} t} z_{2}, e^{i p_{3} t} z_{3}\right)
$$

with period $2 \pi$. Demanding that the stabilisers be trivial one again obtains that the $p_{i}$ have to be \pm 1 giving $2^{4}=8$ possibilities which, taking into account the action of the Weyl group, are reduced to two: $p_{1}=p_{2}= \pm p_{3}=$ 1. The resulting quotient is in either case $\mathbb{C P}^{2}$ (although with opposite orientations) which, as recalled in Section 5.1, is not spin, whence these reductions do not give rise to any geometrically realised supersymmetry. The same conclusion can be reached by computing the weights of $\xi_{S}$ on the representation

$$
\left(\Delta^{2,4} \otimes \Delta^{6,0}\right) \oplus\left(\bar{\Delta}^{2,4} \otimes \bar{\Delta}^{6,0}\right)
$$

of (complexified) Killing spinors, where the bar denotes complex conjugation. From now on we will adopt the notation $\Delta_{+}^{2,4}$ and $\Delta_{+}^{6,0}$ for $\Delta^{2,4}$ and $\Delta^{6,0}$, respectively, and $\Delta_{-}^{2,4}$ and $\Delta_{-}^{6,0}$ for the complex conjugate representations. The weights are $\pm \frac{3}{2}$ and $\pm \frac{1}{2}$ with multiplicities 4 and 12, respectively. This calculation serves to illustrate the general discussion of Section 5 about the fact that not only is $\mathbb{C P}^{2}$ not spin, but indeed all spinors in the quotient spacetime are charged under the circle subgroup associated with the compact dimension.

We now turn our attention to the eleven possible families of reductions where the anti-de Sitter component of the Killing vector is present.

6.3.2 $\xi=\xi_{A}^{(I)}+\xi_{S}$, for $I=4,16$

Both of these cases can be treated simultaneously since (4) is a specialisation of (16) where one of the angles of rotation in $\mathrm{AdS}_{5}$ is put to zero. The analysis 
is very similar to the one above: it shows that for $j=1,2, \varphi_{j} T=2 \pi n_{j}$ for some integers $n_{j}$ which are coprime to the $p_{i}$, whence after rescaling the Killing vector it becomes

$$
\xi=n_{1} \boldsymbol{e}_{34}+n_{2} \boldsymbol{e}_{56}+p_{1} R_{12}+p_{2} R_{34}+p_{3} R_{56} .
$$

As before, the triviality of the stabilisers demands that $p_{i}= \pm 1$, and hence rescaling $\xi$ and up to conjugation by the Weyl group one has

$$
\xi^{ \pm}=n_{1} \boldsymbol{e}_{34}+n_{2} \boldsymbol{e}_{56}+R_{12}+R_{34} \pm R_{56} \quad \text { with } n_{1} \geq n_{2}=0,1,2, \ldots
$$

The case where $n_{1}=n_{2}=0$ was treated above, so we will assume that they cannot both be zero. Let us once again employ the convention that $\gamma_{i}$ denote the $\gamma$-matrices for $\mathrm{C} \ell(2,4)$ and $\Gamma_{a}$ denote those of $\mathrm{C} \ell(6)$. Then the element in $\mathfrak{s o}(2,4) \oplus \mathfrak{s o}(6) \subset \mathrm{C} \ell(2,10)$ corresponding to $\xi$ is given by

$$
X=n_{1} \gamma_{34}+n_{2} \gamma_{56}+\Gamma_{12}+\Gamma_{34} \pm \Gamma_{56} .
$$

Exponentiating this element we get

$$
\begin{aligned}
& \widehat{g}(t)=\exp \frac{t X}{2}=\left(\nVdash \cos \frac{n_{1} t}{2}+\gamma_{34} \sin \frac{n_{1} t}{2}\right)\left(\nVdash \cos \frac{n_{2} t}{2}+\gamma_{56} \sin \frac{n_{2} t}{2}\right) \\
& \times\left(\nVdash \cos \frac{t}{2}+\Gamma_{12} \sin \frac{t}{2}\right)\left(\nVdash \cos \frac{t}{2}+\Gamma_{34} \sin \frac{t}{2}\right)\left(\nVdash \cos \frac{t}{2} \pm \Gamma_{56} \sin \frac{t}{2}\right)
\end{aligned}
$$

and this can only possibly be $-\nVdash$ at $t=2 \pi$, where we get

$$
\widehat{g}(2 \pi)=(-1)^{n_{1}+n_{2}+1} \nVdash ;
$$

whence only for $n_{1}+n_{2}$ odd do we have a spin structure in the quotient.

In order to analyse the amount of supersymmetry preserved by this set of quotients, we shall the action of $X$ on the Killing spinors. The weights of $X$ in that representation are given by

$$
\pm \frac{n_{1}}{2} \pm \frac{n_{2}}{2} \pm \frac{3}{2} \quad \text { and } \quad \pm \frac{n_{1}}{2} \pm \frac{n_{2}}{2} \pm \frac{1}{2}
$$

with uncorrelated signs and with multiplicities 1 and 3, respectively.

It is now straightforward to find out for which values of $n_{1} \geq n_{2}=$ $0,1,2, \ldots$ are there zero weights and how many. One finds the following values for $\left(n_{1}, n_{2}\right)$ :

- $(1,0)$ with 12 zero weights;

- $(2,1)$ with 8 ; 
- $(n+1, n>1)$ with 6 ;

- $(3,0)$ with 4 ; and

- $(n+3, n>0)$ with 2 .

The resulting quotients have fractions $\nu=\frac{3}{8}, \nu=\frac{1}{4}, \nu=\frac{3}{16}, \nu=\frac{1}{8}$ and $\nu=\frac{1}{16}$, respectively.

\subsection{3 $\xi=\xi_{A}^{(I)}+\xi_{S}$, for $I=5,17$}

These cases can again be treated simultaneously because (5) is the specialisation of (17) in which the angle of the spatial rotation is put to zero. Just as in the case of $\mathrm{AdS}_{4} \times S^{7}$ this case does no lead to any smooth reductions due to some points having nontrivial stabiliser: there are non-periodic orbits, whence $\xi$ integrates to an $\mathbb{R}$-action, but there exist periodic orbits at whose points the stabiliser is not trivial.

\subsection{4 $\xi=\xi_{A}^{(I)}+\xi_{S}$ for $I=6,21,20$}

These cases are all specialisations of (20) where one or both of the rotation angles in the AdS space are put to zero.

The Killing vector is

$\xi=-\boldsymbol{e}_{12}-\boldsymbol{e}_{13}+\boldsymbol{e}_{24}+\boldsymbol{e}_{34}+\varphi_{1}\left(\boldsymbol{e}_{12}+\boldsymbol{e}_{34}\right)+\varphi_{2} \boldsymbol{e}_{56}+\theta_{1} R_{12}+\theta_{2} R_{34}+\theta_{3} R_{56}$,

with $\varphi_{2} \geq\left|\varphi_{1}\right|>0$ and $\theta_{i}>\left|\varphi_{1}\right|$ for all $i=1,2,3$. The anti-de Sitter component breaks up into two commuting vector fields: $-\boldsymbol{e}_{12}-\boldsymbol{e}_{13}+\boldsymbol{e}_{24}+\boldsymbol{e}_{34}$ and $\varphi_{1}\left(\boldsymbol{e}_{12}+\boldsymbol{e}_{34}\right)+\varphi_{2} \boldsymbol{e}_{56}$; whence the combined vector field integrates to the following $\mathbb{R}$-action on $\mathbb{R}^{2,4}$ :

$$
\left(\begin{array}{c}
x^{1} \\
x^{2} \\
x^{3} \\
x^{4} \\
x^{5} \\
x^{6}
\end{array}\right) \mapsto\left(\begin{array}{c}
x^{1} \cos \varphi t+x^{2} \sin \varphi t+t\left(\left(x^{1}-x^{4}\right) \sin \varphi t+\left(-x^{2}+x^{3}\right) \cos \varphi t\right) \\
x^{2} \cos \varphi t-x^{1} \sin \varphi t+t\left(\left(x^{1}-x^{4}\right) \cos \varphi t+\left(x^{2}-x^{3}\right) \sin \varphi t\right) \\
x^{3} \cos \varphi t-x^{4} \sin \varphi t+t\left(\left(x^{1}-x^{4}\right) \cos \varphi t+\left(x^{2}-x^{3}\right) \sin \varphi t\right) \\
x^{4} \cos \varphi t+x^{3} \sin \varphi t+t\left(\left(x^{1}-x^{4}\right) \sin \varphi t+\left(-x^{2}+x^{3}\right) \cos \varphi t\right) \\
x^{5} \cos \varphi_{2} t-x^{6} \sin \varphi_{2} t \\
x^{6} \cos \varphi_{2} t+x^{5} \sin \varphi_{2} t
\end{array}\right)
$$

It is clear that there are no fixed points and that the stabilisers are trivial in the quadric and hence also in the universal cover $\mathrm{AdS}_{5}$, whence the quotient 
is smooth and, since $\Gamma \cong \mathbb{R}$, also spin. Notice that in case (6), when both $\varphi_{1}=\varphi_{2}=0$ the resulting orbits are straight lines ruling the quadric.

The action of $\xi$ on the (complexified) Killing spinors breaks up into a nilpotent piece

$$
N=-\gamma_{12}-\gamma_{13}+\gamma_{24}+\gamma_{34}
$$

and a semisimple piece

$$
S=\varphi_{1}\left(\gamma_{12}+\gamma_{34}\right)+\varphi_{2} \gamma_{56}+\theta_{1} \Gamma_{12}+\theta_{2} \Gamma_{34}+\theta_{3} \Gamma_{56},
$$

whence the invariant Killing spinors lie in the kernel of both $N$ and $S$. The analysis of the kernel of $N$ is very similar to the one carried out in Section 6.1.5. Indeed, $N$ is essentially what we called $N^{+}$there. Let us decompose

$$
\Delta_{ \pm}^{2,4}=\left(\Delta_{+}^{2,2} \otimes \Delta_{ \pm}^{0,2}\right) \oplus\left(\Delta_{-}^{2,2} \otimes \Delta_{\mp}^{0,2}\right)
$$

and observe that as in Section 6.1.5, $N$ acts trivially in $\Delta_{-}^{2,2}$ and has a onedimensional kernel in $\Delta_{+}^{2,2}$ on which the term $\varphi_{1}\left(\gamma_{12}+\gamma_{34}\right)$ acts trivially. It is then easy to compute the weights of $S$ on the kernel of $N$. They are given by the weights of

$$
\varphi_{2} \gamma_{56}+\theta_{1} \Gamma_{12}+\theta_{2} \Gamma_{34}+\theta_{3} \Gamma_{56}
$$

acting on

$$
\left(\Delta_{+}^{0,2} \otimes \Delta_{+}^{6,0}\right) \oplus\left(\Delta_{-}^{0,2} \otimes \Delta_{-}^{6,0}\right)
$$

and the weights of $S$ acting on

$$
\left(\Delta_{-}^{2,2} \otimes \Delta_{-}^{0,2} \otimes \Delta_{+}^{6,0}\right) \oplus\left(\Delta_{-}^{2,2} \otimes \Delta_{+}^{0,2} \otimes \Delta_{-}^{6,0}\right) .
$$

The calculation is routine and we obtain the following 24 weights

$$
\begin{array}{r} 
\pm \frac{1}{2} \varphi_{2} \pm \frac{1}{2} \theta_{1} \pm \frac{1}{2} \theta_{2} \pm \frac{1}{2} \theta_{3} \\
\varphi_{1} \pm \frac{1}{2} \varphi_{2} \pm \frac{1}{2} \theta_{1} \pm \frac{1}{2} \theta_{2} \pm \frac{1}{2} \theta_{3} \\
-\varphi_{1} \pm \frac{1}{2} \varphi_{2} \pm \frac{1}{2} \theta_{1} \pm \frac{1}{2} \theta_{2} \pm \frac{1}{2} \theta_{3}
\end{array}
$$

where the product of the signs is always negative for a total of 8 weights per line. These 24 weights define 12 hyperplanes. For parameters lying in one and only one such hyperplane there are two invariant Killing spinors. Three of the hyperplanes contain no points due to the conditions on the parameters coming from causality and the ordering due to the action of the Weyl group. The other nine hyperplanes are defined by the conditions:

$$
\begin{aligned}
\varphi_{2} \pm\left(\theta_{1}-\theta_{2}\right)+\theta_{3} & =0 \\
\varphi_{2}-\theta_{1}-\theta_{2}-\theta_{3} & =0 \\
\varphi_{2} \pm 2 \varphi_{1}-\theta_{1}-\theta_{2}+\theta_{3} & =0 \\
\varphi_{2} \pm 2 \varphi_{1} \pm\left(\theta_{1}-\theta_{2}\right)-\theta_{3} & =0
\end{aligned}
$$


where the signs in the last line are uncorrelated. Each of these reductions preserve a fraction $\nu=\frac{1}{16}$ of the ten-dimensional supersymmetry.

The analysis of the possible intersections of these hyperplanes has been done with the help of MathematicA. The following conditions, where each line is one condition, guarantee that the parameters lie in the intersection of precisely two hyperplanes:

$$
\begin{aligned}
& \theta_{1}=\theta_{2} \quad \theta_{3}=-\varphi_{2} \\
& \theta_{1}=\theta_{2} \quad \theta_{3}= \pm 2 \varphi_{1}+\varphi_{2} \\
& \theta_{1}=\varphi_{2} \quad \theta_{2}=-\theta_{3} \\
& \theta_{1} \pm \varphi_{1}=\theta_{2} \pm \theta_{3} \quad \varphi_{2}=-\varphi_{1} \\
& \theta_{1} \pm \varphi_{1}=\theta_{2} \mp \theta_{3} \quad \varphi_{2}=\varphi_{1} \\
& \theta_{1}+\varphi_{1}=-\theta_{2}-\theta_{3} \quad \varphi_{2}= \pm \varphi_{1} \\
& \theta_{1}=\theta_{2} \pm 2 \varphi_{1} \quad \theta_{3}=\varphi_{2} \\
& \theta_{1}=\theta_{3} \pm 2 \varphi_{1} \quad \theta_{2}=\varphi_{2} \\
& \theta_{1}=\varphi_{2} \pm 2 \varphi_{1} \quad \theta_{2}=\theta_{3} \\
& \theta_{2}=\theta_{3} \pm 2 \varphi_{1} \quad \theta_{1}=\varphi_{2} \text {. }
\end{aligned}
$$

Each of these reductions preserve $\frac{1}{8}$ of the ten-dimensional supersymmetry.

Similarly, parameters satisfying the following conditions lie in precisely three hyperplanes:

$$
\begin{aligned}
\theta_{1} & =\theta_{2}= \pm \theta_{3}=\varphi_{2} \\
\theta_{1} & =\theta_{2}=\theta_{3}= \pm 2 \varphi_{1}+\varphi_{2} \\
\theta_{1} & =\theta_{2}=\theta_{3} \pm 2 \varphi_{1}=\varphi_{2} \\
\theta_{1} \pm 2 \varphi_{1} & =\theta_{2}=\theta_{3}=\varphi_{2} .
\end{aligned}
$$

Each of these reductions preserves $\frac{3}{16}$ of the supersymmetry. The causality constraints on the parameters, which do not allow them to vanish, forbid intersections of four or more hyperplanes.

\subsection{5 $\xi=\xi_{A}^{(I)}+\xi_{S}$ for $I=11,25$}

These two cases are treated simultaneously because (11) is the specialisation of $(25)$ where $\varphi=0$. The Killing vector is

$$
\xi=\beta\left(\boldsymbol{e}_{13}+\boldsymbol{e}_{24}\right)+\varphi \boldsymbol{e}_{56}+\theta_{1} R_{12}+\theta_{2} R_{34}+\theta_{3} R_{56},
$$

where $\varphi \geq 0$ and where $\theta_{1} \geq \theta_{2} \geq\left|\theta_{3}\right|$ are now allowed to be zero provided that $\beta$ is not. The case with $\beta=0$ was treated before, so we will assume 
$\beta \neq 0$ and hence rescale $\xi$ such that $\beta=1$. The anti-de Sitter component integrates to the following action on $\mathbb{R}^{2,4}$ :

$$
\left(\begin{array}{l}
x^{1} \\
x^{2} \\
x^{3} \\
x^{4} \\
x^{5} \\
x^{6}
\end{array}\right) \mapsto\left(\begin{array}{l}
x^{1} \cosh t-x^{3} \sinh t \\
x^{2} \cosh t-x^{4} \sinh t \\
x^{3} \cosh t-x^{1} \sinh t \\
x^{4} \cosh t-x^{2} \sinh t \\
x^{5} \cos \varphi t-x^{6} \sin \varphi t \\
x^{6} \cos \varphi t+x^{5} \sin \varphi t
\end{array}\right)
$$

which is clearly a free action of $\Gamma \cong \mathbb{R}$ on the quadric and hence on $\operatorname{AdS}_{5} \times S^{5}$ for all values of $\theta_{i}$. The resulting quotient is therefore smooth and, since $\Gamma \cong \mathbb{R}$, also spin.

To analyse the supersymmetry, notice that the action of $\xi$ on the (complexified) Killing spinors breaks up into two commuting semisimple pieces:

$$
S_{1}=\gamma_{13}+\gamma_{24}
$$

having real eigenvalues, and

$$
S_{2}=\varphi \gamma_{56}+\theta_{1} \Gamma_{12}+\theta_{2} \Gamma_{34}+\theta_{3} \Gamma_{56}
$$

having imaginary eigenvalues. Therefore a Killing spinor is invariant under $\xi$ if and only if it is simultaneously annihilated by $S_{1}$ and $S_{2}$. The kernel of $S_{1}$ consists of those spinors satisfying $\gamma_{1234} \varepsilon=\varepsilon$, or equivalently $\gamma_{56} \varepsilon= \pm i \varepsilon$ in $\Delta_{ \pm}^{2,4}$. The weights of $S_{2}$ on the kernel of $S_{1}$ are easy to compute, and one obtains

$$
\pm \frac{1}{2} \varphi \pm \frac{1}{2} \theta_{1} \pm \frac{1}{2} \theta_{2} \pm \frac{1}{2} \theta_{3}
$$

with uncorrelated signs whose product is negative. Their vanishing determines four hyperplanes:

$$
\begin{aligned}
& \varphi+\theta_{1}+\theta_{2}-\theta_{3}=0 \\
& \varphi-\theta_{1}-\theta_{2}-\theta_{3}=0 \\
& \varphi+\theta_{1}-\theta_{2}+\theta_{3}=0 \\
& \varphi-\theta_{1}+\theta_{2}+\theta_{3}=0 .
\end{aligned}
$$

Whenever the parameters are such that they lie on precisely one of these hyperplanes, the reduction preserves four supersymmetries. These reductions preserve $\frac{1}{8}$ of the supersymmetry. This happens whenever the second, third or fourth equations are satisfied, since the first equation implies that $\varphi=\theta_{1}=\theta_{2}=\theta_{3}=0$ due to the constraints on these parameters, and these values satisfy all four equations. 
Whenever the following conditions are satisfied, the parameters belong to precisely two such hyperplanes and the reduction preserves $\frac{1}{4}$ of the supersymmetry:

$$
\begin{gathered}
\varphi=\theta_{1} \neq \theta_{2}=-\theta_{3} \\
\theta_{1}=\theta_{2} \neq \varphi=-\theta_{3} .
\end{gathered}
$$

Similarly, whenever the condition

$$
\theta_{1}=\theta_{2}=\varphi=-\theta_{3} \neq 0
$$

are met, the parameters belong to precisely three such hyperplanes and the reduction preserves $\frac{3}{8}$ of the supersymmetry. Finally, if all the parameters vanish, we obtain a half-BPS reduction.

6.3.6 $\xi=\xi_{A}^{(23)}+\xi_{S}$

The Killing vector in this case is

$$
\xi=\boldsymbol{e}_{13}-\boldsymbol{e}_{34}+\boldsymbol{e}_{25}-\boldsymbol{e}_{56}+\theta_{1} R_{12}+\theta_{2} R_{34}+\theta_{3} R_{56} .
$$

The anti-de Sitter component integrates to the following $\mathbb{R}$-action on $\mathbb{R}^{2,4}$ :

$$
\left(\begin{array}{c}
x^{1} \\
x^{2} \\
x^{3} \\
x^{4} \\
x^{5} \\
x^{6}
\end{array}\right) \mapsto\left(\begin{array}{c}
x^{1}-t x^{3}+\frac{1}{2} t^{2}\left(x^{1}-x^{4}\right) \\
x^{2}-t x^{5}+\frac{1}{2} t^{2}\left(x^{2}-x^{6}\right) \\
x^{3}+t\left(x^{4}-x^{1}\right) \\
x^{4}-t x^{3}+\frac{1}{2} t^{2}\left(x^{1}-x^{4}\right) \\
x^{5}+t\left(x^{6}-x^{2}\right) \\
x^{6}-t x^{5}+\frac{1}{2} t^{2}\left(x^{2}-x^{6}\right)
\end{array}\right)
$$

which is clearly free of fixed points on $\mathrm{AdS}_{5}$ and has trivial stabilisers. The resulting quotient $\left(\operatorname{AdS}_{5} \times S^{5}\right) / \Gamma$ is therefore smooth and, since $\Gamma \cong \mathbb{R}$, it is also spin.

To analyse the supersymmetry of this class of quotients, notice that the action of $\xi$ on the (complexified) Killing spinors splits into two commuting pieces: one nilpotent,

$$
N=\gamma_{13}-\gamma_{34}+\gamma_{25}-\gamma_{56}
$$

and one semisimple

$$
S=\theta_{1} \Gamma_{12}+\theta_{2} \Gamma_{34}+\theta_{3} \Gamma_{56} .
$$

The nilpotent operator is the spinorial representation of a double null rotation and we will now show that it preserves one-half of the AdS supersymmetry. Let us rewrite $N$ as

$$
N=\left(\gamma_{1}+\gamma_{4}\right) \gamma_{3}+\left(\gamma_{2}+\gamma_{6}\right) \gamma_{5},
$$


which suggests the following decomposition of the spinorial representations $\Delta_{ \pm}^{2,4}$

$$
\Delta_{ \pm}^{2,4} \cong V_{ \pm} \oplus\left(\gamma_{1}+\gamma_{4}\right) V_{\mp} \oplus\left(\gamma_{2}+\gamma_{6}\right) V_{\mp} \oplus\left(\gamma_{1}+\gamma_{4}\right)\left(\gamma_{2}+\gamma_{6}\right) V_{ \pm}
$$

where $V_{ \pm} \subset \Delta_{ \pm}^{2,4}$ is the subspace

$$
V_{ \pm}=\operatorname{ker}\left(\gamma_{1}-\gamma_{4}\right) \cap \operatorname{ker}\left(\gamma_{2}-\gamma_{6}\right)
$$

This decomposition is nothing else but the decomposition of the Clifford modules $\Delta_{ \pm}^{2,4}$ as fermionic Fock spaces associated to the annihilation and creation operators $\gamma_{1} \pm \gamma_{4}$ and $\gamma_{2} \pm \gamma_{5}$, with $V_{ \pm}$the one-dimensional subspace spanned by the Clifford vacua. Accordingly, every $\varepsilon \in \Delta_{ \pm}^{2,4}$ has a unique decomposition of the form

$$
\varepsilon=\varepsilon_{1}+\left(\gamma_{1}+\gamma_{4}\right) \varepsilon_{2}+\left(\gamma_{2}+\gamma_{6}\right) \varepsilon_{3}+\left(\gamma_{1}+\gamma_{4}\right)\left(\gamma_{2}+\gamma_{6}\right) \varepsilon_{4},
$$

where $\varepsilon_{1}, \varepsilon_{4} \in V_{ \pm}$and $\varepsilon_{2}, \varepsilon_{3} \in V_{\mp}$. It is now clear that

$$
N \varepsilon=0 \Longleftrightarrow \varepsilon_{1}=0 \text { and } \varepsilon_{3}=\gamma_{35} \varepsilon_{2} .
$$

Notice that if $\varepsilon \in V_{ \pm}$then $\gamma_{35} \varepsilon= \pm i \varepsilon$, whence we can write the most general spinor $\varepsilon \in \Delta_{ \pm}^{2,4}$ in the kernel of $N$ as

$$
\varepsilon=\left(\left(\gamma_{1}+\gamma_{4}\right) \mp i\left(\gamma_{2}+\gamma_{6}\right)\right) \varepsilon_{2}+\left(\gamma_{1}+\gamma_{4}\right)\left(\gamma_{2}+\gamma_{6}\right) \varepsilon_{4}
$$

whence ker $N \subset \Delta_{ \pm}^{2,4}$ is two-dimensional.

The calculation of weights of the semisimple piece $S$ on $\operatorname{ker} N$ is routine, and we obtain the following eight weights,

$$
\pm \frac{1}{2} \theta_{1} \pm \frac{1}{2} \theta_{2} \pm \frac{1}{2} \theta_{3}
$$

with uncorrelated signs and each with multiplicity 2 . Supersymmetry requires the existence of zero weights. These conditions determine four hyperplanes

$$
\theta_{1} \pm \theta_{2} \pm \theta_{3}=0
$$

and if the parameters lie in precisely one of these hyperplanes, the resulting quotient has four supersymmetries. Two of the hyperplanes have no points due to the conditions on the parameters. The remaining two hyperplanes are

$$
\theta_{1}-\theta_{2} \pm \theta_{3}=0
$$

and the resulting quotients preserve a fraction $\nu=\frac{1}{8}$ of the supersymmetry. The intersection of these two hyperplanes consists of parameters satisfying

$$
\theta_{1}=\theta_{2} \quad \text { and } \quad \theta_{3}=0 .
$$


Such reductions preserve $\frac{1}{4}$ of the supersymmetry. Finally, if $\theta_{i}=0$ then the reduction preserves one half of the supersymmetry. The discrete quotient associated to this last case was discussed in $[36]^{1}$

\subsection{7 $\xi=\xi_{A}^{(24)}+\xi_{S}$}

In this case we are adding a timelike rotation to the case $\xi_{A}^{(16)}$ treated above, but in the simply-connected $\mathrm{AdS}_{5}$ these orbits are not periodic. Therefore this integrates to an action of $\Gamma \cong \mathbb{R}$ and as there are no points on which $\xi_{A}^{(24)}=0$, the stabilisers are all trivial. The most general spacelike smooth reduction of this type corresponds to the Killing vector

$$
\xi=\boldsymbol{e}_{12}+\varphi_{1} \boldsymbol{e}_{34}+\varphi_{2} \boldsymbol{e}_{56}+\theta_{1} R_{12}+\theta_{2} R_{34}+\theta_{3} R_{56}
$$

where we have relabelled the $\varphi_{i}$ and rescaled the vector and where $\theta_{1} \geq \theta_{2} \geq$ $\theta_{3}>1$ and $\varphi_{1} \geq \varphi_{2} \geq 1$. Since $\Gamma \cong \mathbb{R}$, the quotient is spin.

To analyse the supersymmetry of this class of quotients, we notice that the action of $\xi$ on the (complexified) Killing spinors is semisimple with weights:

$$
\pm \frac{1}{2} \varphi_{1} \pm \frac{1}{2} \varphi_{2} \pm \frac{1}{2} \varphi_{3} \pm \frac{1}{2} \theta_{1} \pm \frac{1}{2} \theta_{2} \pm \frac{1}{2} \theta_{3}
$$

where the signs are uncorrelated except that their product is positive and where we have reintroduced $\varphi_{3}$, which can be set to 1 as was done before if desired. Supersymmetry requires the vanishing of one or more of these weights. These conditions define 16 hyperplanes, of which 6 have no points for our choice of ordering of the parameters. If the parameters lie in precisely one of the remaining 10 hyperplanes, the corresponding quotient has two supersymmetries. This happens whenever one of the following 10 equations is satisfied:

$$
\begin{aligned}
\varphi_{1} \pm\left(\varphi_{2}-\varphi_{3}\right)-\theta_{1} \pm\left(\theta_{2}+\theta_{3}\right) & =0 \\
\varphi_{1} \pm\left(\varphi_{2}+\varphi_{3}\right)+\theta_{1}-\theta_{2}-\theta_{3} & =0 \\
\varphi_{1} \pm\left(\varphi_{2}+\varphi_{3}\right)-\theta_{1} \pm\left(\theta_{2}-\theta_{3}\right) & =0 .
\end{aligned}
$$

Such reductions preserve a fraction $\nu=\frac{1}{16}$ of the supersymmetry.

The analysis of the possible intersections of these hyperplanes has again been done with the help of Mathematica. The following 16 conditions

\footnotetext{
${ }^{1}$ It was wrongly argued in [36] that the supersymmetry of the double null rotation quotient was $\nu=\frac{1}{4}$.
} 
guarantee that the parameters lie in the intersection of precisely two hyperplanes:

$$
\begin{array}{ll}
\varphi_{2}=\varphi_{3} & \theta_{1}=\varphi_{1} \pm\left(\theta_{2}+\theta_{3}\right) \\
\theta_{1}=\varphi_{1} & \theta_{2}=\varphi_{2} \pm\left(\theta_{3}+\varphi_{3}\right) \\
\theta_{2}=\varphi_{2} & \theta_{1}=\varphi_{1} \pm\left(\theta_{3}+\varphi_{3}\right) \\
\theta_{3}=\varphi_{2} & \theta_{1}=\varphi_{1} \pm\left(\theta_{2}+\varphi_{3}\right) \\
\theta_{1}=\theta_{2} & \theta_{3}=\varphi_{1} \pm\left(\varphi_{2}+\varphi_{3}\right) \\
\theta_{2}=\theta_{3} & \theta_{1}=\varphi_{1} \pm\left(\varphi_{2}+\varphi_{3}\right) \\
\varphi_{1}=\varphi_{2} & \theta_{1}=\theta_{2}+\theta_{3}+\varphi_{3} \\
\theta_{1}=\varphi_{2} & \varphi_{1}=\theta_{2}+\theta_{3}+\varphi_{3} \\
\theta_{2}=\varphi_{1} & \theta_{1}=\theta_{3}+\varphi_{2}+\varphi_{3} \\
\theta_{3}=\varphi_{1} & \theta_{1}=\theta_{2}+\varphi_{2}+\varphi_{3} .
\end{array}
$$

Each of these reductions preserve $\frac{1}{8}$ of the ten-dimensional supersymmetry.

Similarly, parameters satisfying the following 13 conditions lie in precisely three hyperplanes:

$$
\begin{aligned}
& \varphi_{1}=\varphi_{2}=\varphi_{3}=\theta_{1}-\theta_{2}-\theta_{3} \\
& \theta_{1}=\theta_{2}=\theta_{3}=\varphi_{1} \pm\left(\varphi_{2}+\varphi_{3}\right) \\
& \theta_{1}=\varphi_{1}=\varphi_{2}=\theta_{2}+\theta_{3}+\varphi_{3} \\
& \theta_{3}=\varphi_{1}=\varphi_{2}=\theta_{1}-\theta_{2}-\varphi_{3} \\
& \theta_{2}=\varphi_{1}=\varphi_{2}=\theta_{1}-\theta_{3}-\varphi_{3} \\
& \theta_{1}=\theta_{2}=\varphi_{2}=\varphi_{1}-\theta_{3}-\varphi_{3} \\
& \theta_{2}=\theta_{3}=\varphi_{2}= \pm\left(\theta_{1}-\varphi_{1}\right)-\varphi_{3} \\
& \theta_{1}=\theta_{2}=\varphi_{1}=\theta_{3} \pm\left(\varphi_{2}+\varphi_{3}\right) \\
& \theta_{2}=\theta_{3}=\varphi_{1}=\theta_{1} \pm\left(\varphi_{2}+\varphi_{3}\right) .
\end{aligned}
$$

Each of these reductions preserves $\frac{3}{16}$ of the supersymmetry.

Finally there are reductions preserving $\frac{1}{4}$ of the supersymmetry corresponding to parameters lying in precisely four of the hyperplanes, which happens whenever either one of the two conditions hold:

$$
\begin{array}{ll}
\theta_{1}=2 \varphi_{2}+\varphi_{3} & \theta_{2}=\theta_{3}=\varphi_{1}=\varphi_{2} \\
\varphi_{1}=2 \varphi_{2}+\varphi_{3} & \theta_{1}=\theta_{2}=\theta_{3}=\varphi_{2}
\end{array}
$$

The causality constraints on the parameters, which do not allow them to vanish, forbid intersections of five or more hyperplanes. 


\subsubsection{Summary}

The smooth spacelike supersymmetric quotients of $\mathrm{AdS}_{5} \times S^{5}$ are summarised in Table 8, where we have also indicated the fraction $\nu$ of the IIB supersymmetry preserved by the quotient.

\begin{tabular}{|c|c|c|c|}
\hline \multirow[b]{2}{*}{ Killing vector } & \multicolumn{2}{|c|}{ Conditions for } & \multirow[b]{2}{*}{$\nu$} \\
\hline & Causality/Weyl & Supersymmetry & \\
\hline $\begin{aligned} \varphi_{1} \boldsymbol{e}_{34} & +\varphi_{2} \boldsymbol{e}_{56} \\
& +R_{12}+R_{34} \pm R_{56}\end{aligned}$ & $\varphi_{1} \geq \varphi_{2} \geq 0$ & $\begin{array}{l}\varphi_{1}=1, \varphi_{2}=0 \\
\varphi_{1}=2, \varphi_{2}=1 \\
\varphi_{1}=1+n, \varphi_{2}=n>1 \\
\varphi_{1}=3, \varphi_{2}=0 \\
\varphi_{1}=3+n, \varphi_{2}=n>0\end{array}$ & $\begin{array}{l}\frac{3}{8} \\
\frac{1}{4} \\
\frac{3}{16} \\
\frac{1}{8} \\
\frac{1}{16}\end{array}$ \\
\hline $\begin{array}{l}-\boldsymbol{e}_{12}-\boldsymbol{e}_{13}+\boldsymbol{e}_{24}+\boldsymbol{e}_{34} \\
+\varphi_{1}\left(\boldsymbol{e}_{12}+\boldsymbol{e}_{34}\right)+\varphi_{2} \boldsymbol{e}_{56} \\
+\theta_{1} R_{12}+\theta_{2} R_{34}+\theta_{3} R_{56}\end{array}$ & $\begin{array}{l}\varphi_{2} \geq\left|\varphi_{1}\right|>0 \\
\theta_{1} \geq \theta_{2} \geq\left|\theta_{3}\right|>\left|\varphi_{1}\right|\end{array}$ & $\begin{array}{l}(6.6) \\
(6.7) \\
(6.8)\end{array}$ & $\begin{array}{l}\frac{1}{16} \\
\frac{1}{8} \\
\frac{3}{16} \\
\end{array}$ \\
\hline $\begin{array}{l}\boldsymbol{e}_{13}+\boldsymbol{e}_{24}+\varphi \boldsymbol{e}_{56} \\
+\theta_{1} R_{12}+\theta_{2} R_{34}+\theta_{3} R_{56}\end{array}$ & $\begin{array}{l}\theta_{1} \geq \theta_{2} \geq\left|\theta_{3}\right| \\
\varphi \geq 0\end{array}$ & $\begin{aligned} &(6.9) \\
&(6.10) \\
& \varphi= \theta_{1}= \\
& \theta_{2}=-\theta_{3} \neq 0 \\
& \theta_{i}=\varphi=0\end{aligned}$ & $\begin{array}{l}\frac{1}{8} \\
\frac{1}{4} \\
\frac{3}{8} \\
\frac{1}{2} \\
\end{array}$ \\
\hline $\begin{array}{l}\boldsymbol{e}_{13}-\boldsymbol{e}_{34}+\boldsymbol{e}_{25}-\boldsymbol{e}_{56} \\
+\theta_{1} R_{12}+\theta_{2} R_{34}+\theta_{3} R_{56}\end{array}$ & $\theta_{1} \geq \theta_{2} \geq\left|\theta_{3}\right|$ & $\begin{array}{l}\theta_{1}-\theta_{2} \pm \theta_{3}=0 \\
\theta_{1}=\theta_{2}, \theta_{3}=0 \\
\theta_{i}=0\end{array}$ & $\begin{array}{l}\frac{1}{8} \\
\frac{1}{4} \\
\frac{1}{2} \\
\end{array}$ \\
\hline $\begin{array}{l}\boldsymbol{e}_{12}+\varphi_{1} \boldsymbol{e}_{34}+\varphi_{2} \boldsymbol{e}_{56} \\
+\theta_{1} R_{12}+\theta_{2} R_{34}+\theta_{3} R_{56}\end{array}$ & $\begin{array}{l}\varphi_{1} \geq \varphi_{2} \geq 1 \\
\theta_{1} \geq \theta_{2} \geq \theta_{3}>1\end{array}$ & $\begin{array}{l}(6.11) \text { with } \varphi_{3}=1 \\
(6.12) \text { with } \varphi_{3}=1 \\
(6.13) \text { with } \varphi_{3}=1 \\
(6.14) \text { with } \varphi_{3}=1\end{array}$ & $\begin{array}{l}\frac{1}{16} \\
\frac{1}{8} \\
\frac{3}{16} \\
\frac{1}{4}\end{array}$ \\
\hline
\end{tabular}

Table 8: Smooth spacelike supersymmetric quotients of $\mathrm{AdS}_{5} \times S^{5}$ 


\subsection{Supersymmetric quotients of $\mathrm{AdS}_{7} \times S^{4}$}

The $\operatorname{AdS}_{7} \times S^{4}$ vacuum of eleven-dimensional supergravity is such that $S^{4}$ has radius of curvature $R_{S}=R$ and $\mathrm{AdS}_{7}$ has radius of curvature $R_{A}=2 R$. Letting $s>0$ denote the scalar curvature of the sphere, the 4 -form is given by

$$
F=\sqrt{\frac{3}{4} s} \operatorname{dvol}_{S}
$$

where $\mathrm{dvol}_{S}$ is the volume form of $S^{4}$. This vacuum solution has thirty-two supersymmetries. In this section we will study the smooth supersymmetric quotients.

In this case the sphere is even-dimensional, whence every (Killing) vector has zeroes. This means that in contrast to the cases treated previously, we require the component $\xi_{A}$ of the Killing vector tangent to $\mathrm{AdS}_{7}$ to be everywhere spacelike. The results in Section 4.4 tell us that they are the ones labelled by (11), (25) and (39) all three with $\beta_{1}=\beta_{2},(23)$ and (37), which we proceed to investigate.

Throughout this section the spherical component $\xi_{S}$ of the Killing vector is given by

$$
\xi_{S}=\theta_{1} R_{12}+\theta_{2} R_{34}
$$

where we can choose $\theta_{1} \geq \theta_{2} \geq 0$. This vector field clearly vanishes at the points $(0,0,0,0, \pm R)$.

\subsection{1 $\xi=\xi_{A}^{(I)}+\xi_{S}$, for $I=11,25,39$}

These three cases can be treated simultaneously, since they are all specialisations of (39) where one or both of the rotation angles in $\mathrm{AdS}_{7}$ vanish. The Killing vector is given by

$$
\xi=\beta\left(\boldsymbol{e}_{13}+\boldsymbol{e}_{24}\right)+\varphi_{1} \boldsymbol{e}_{56}+\varphi_{2} \boldsymbol{e}_{78}+\theta_{1} R_{12}+\theta_{2} R_{34},
$$

where $\beta>0$, whence we can set it equal to 1 by rescaling $\xi$. The action on $\mathbb{R}^{2,6}$ integrates to

$$
\left(\begin{array}{l}
x^{1} \\
x^{2} \\
x^{3} \\
x^{4} \\
x^{5} \\
x^{6} \\
x^{7} \\
x^{8}
\end{array}\right) \mapsto\left(\begin{array}{c}
x^{1} \cosh t-x^{3} \sinh t \\
x^{2} \cosh t-x^{4} \sinh t \\
x^{3} \cosh t-x^{1} \sinh t \\
x^{4} \cosh t-x^{2} \sinh t \\
x^{5} \cos \varphi_{1} t-x^{6} \sin \varphi_{1} t \\
x^{6} \cos \varphi_{1} t+x^{5} \sin \varphi_{1} t \\
x^{7} \cos \varphi_{2} t-x^{8} \sin \varphi_{2} t \\
x^{8} \cos \varphi_{2} t+x^{7} \sin \varphi_{2} t
\end{array}\right)
$$


which is clearly a free action of $\Gamma \cong \mathbb{R}$ on the quadric and hence on $\mathrm{AdS}_{7} \times S^{4}$ for all values of $\theta_{i}$ and $\varphi_{i}$. The resulting background $\left(\operatorname{AdS}_{7} \times S^{4}\right) / \Gamma$ is therefore smooth and, since $\Gamma \cong \mathbb{R}$, also spin.

Let us discuss the supersymmetry preserved by these quotients. As in previous sections, the action of $\xi$ on Killing spinors given by equation (6.1) splits into two commuting semisimple pieces:

$$
S_{1}=\gamma_{13}+\gamma_{24}
$$

and

$$
S_{2}=\varphi_{1} \gamma_{56}+\varphi_{2} \gamma_{78}+\theta_{1} \Gamma_{12}+\theta_{2} \Gamma_{34}
$$

where $\gamma_{i j}$ are the gamma matrices of $\mathrm{C} \ell(2,6)$ and $\Gamma_{i j}$ those of $\mathrm{C} \ell(5,0)$.

The kernel of $S_{1}$ consists of spinors $\varepsilon$ such that

$$
\gamma_{1234} \varepsilon=\varepsilon
$$

which on $\Delta_{-}^{2,6}$ implies the relation

$$
\gamma_{5678} \varepsilon=-\varepsilon
$$

The weights of $S_{2}$ on ker $S_{1} \otimes \Delta^{5,0} \subset \Delta_{-}^{2,6} \otimes \Delta^{5,0}$ are easily computed to be

$$
\pm \frac{1}{2}\left(\varphi_{1}+\varphi_{2}\right) \pm \frac{1}{2} \theta_{1} \pm \frac{1}{2} \theta_{2}
$$

with uncorrelated signs and each with multiplicity 2. The zero-weight conditions give four linear equations on the parameters. If one (and only one) of these equations is satisfied, the corresponding quotient preserves four supersymmetries. There are cases in which one or more equations can be satisfied simultaneously and there is enhancement of supersymmetry. This is easily analysed yielding the following supersymmetric quotients:

- $\varphi_{1}+\varphi_{2}=\theta_{1} \pm \theta_{2}$, with 4 supersymmetries;

- $\varphi_{1}+\varphi_{2}=0$ and $\theta_{1}=\theta_{2} \neq 0$, with 8 supersymmetries; and

- $\varphi_{1}+\varphi_{2}=0$ and $\theta_{1}=\theta_{2}=0$, with 16 supersymmetries.

Note that the last supersymmetric quotient is another example of the phenomenon first encountered in quotients of $\mathrm{AdS}_{3} \times S^{3}$ in which, supersymmetry wise, one can deform the action of the quotient in a non-trivial way for free, that is, without decreasing the amount of supersymmetry preserved by the quotient. 
6.4.2 $\xi=\xi_{A}^{(I)}+\xi_{S}$, for $I=23,37$

Both of these cases can be treated simultaneously since (23) is the specialisation of (37) where the rotation angle in $\mathrm{AdS}_{7}$ is set to zero. The Killing vector in this case is

$$
\xi=\boldsymbol{e}_{13}-\boldsymbol{e}_{34}+\boldsymbol{e}_{25}-\boldsymbol{e}_{56}+\varphi \boldsymbol{e}_{78}+\theta_{1} R_{12}+\theta_{2} R_{34} .
$$

The anti-de Sitter component integrates to the following $\mathbb{R}$-action on $\mathbb{R}^{2,6}$ :

$$
\left(\begin{array}{c}
x^{1} \\
x^{2} \\
x^{3} \\
x^{4} \\
x^{5} \\
x^{6} \\
x^{7} \\
x^{8}
\end{array}\right) \mapsto\left(\begin{array}{c}
x^{1}-t x^{3}+\frac{1}{2} t^{2}\left(x^{1}-x^{4}\right) \\
x^{2}-t x^{5}+\frac{1}{2} t^{2}\left(x^{2}-x^{6}\right) \\
x^{3}+t\left(x^{4}-x^{1}\right) \\
x^{4}-t x^{3}+\frac{1}{2} t^{2}\left(x^{1}-x^{4}\right) \\
x^{5}+t\left(x^{6}-x^{2}\right) \\
x^{6}-t x^{5}+\frac{1}{2} t^{2}\left(x^{2}-x^{6}\right) \\
x^{7} \cos \varphi t-x^{8} \sin \varphi t \\
x^{8} \cos \varphi t+x^{7} \sin \varphi t
\end{array}\right),
$$

which is clearly free of fixed points on $\mathrm{AdS}_{7}$ and has trivial stabilisers. The resulting quotient is smooth and also spin.

The supersymmetry analysis departs from the observation that the action of $\xi$ on the (complexified) Killing spinors $\Delta_{-}^{2,6} \otimes \Delta^{5,0}$ splits into a nilpotent piece

$$
N=\gamma_{13}-\gamma_{34}+\gamma_{25}-\gamma_{56}
$$

and a semisimple piece

$$
S=\varphi \gamma_{78}+\theta_{1} \Gamma_{12}+\theta_{2} \Gamma_{34}
$$

The invariant Killing spinors are therefore in the intersection of the kernels of both $N$ and $S$. The nilpotent operator $N$ corresponds to a double null rotation and its kernel was analysed already in Section 6.3.6, where we found that it is half-dimensional, independent of chirality. In other words, under the split

$$
\Delta_{-}^{2,6}=\left(\Delta_{+}^{2,4} \otimes \Delta_{+}^{0,2}\right) \oplus\left(\Delta_{-}^{2,4} \otimes \Delta_{-}^{0,2}\right)
$$

the kernels of $N$ restricted to $\Delta_{ \pm}^{2,4}$ are two-dimensional and $S$ acts trivially on them. The weights of $S$ on ker $N$ are therefore those of $S$ restricted to $\Delta^{0,2} \otimes \Delta^{5,0}$ but with multiplicity 2 . These weights are

$$
\pm \frac{1}{2} \varphi \pm \frac{1}{2} \theta_{1} \pm \frac{1}{2} \theta_{2}
$$

with uncorrelated signs. Their vanishing defines four hyperplanes in the parameter space, each contributing four supersymmetries to the supersymmetry of the quotient, with possible enhancement when the parameters lie 
in more than one such hyperplane. The analysis is again routine and yields the following supersymmetric quotients:

- $\varphi=\theta_{1} \pm \theta_{2}$, with four supersymmetries;

- $\varphi=0$ and $\theta_{1}=\theta_{2}$, with eight supersymmetries;

- $\varphi=\theta_{1}$ and $\theta_{2}=0$, with eight supersymmetries; and

- $\varphi=\theta_{i}=0$, with sixteen supersymmetries.

\subsubsection{Summary}

The smooth spacelike supersymmetric quotients of $\mathrm{AdS}_{7} \times S^{4}$ are summarised in Table 9, where we have also indicated the fraction $\nu$ of the eleven-dimensional supersymmetry preserved by the quotient.

\begin{tabular}{|c|l|l|c|}
\hline & \multicolumn{2}{|c|}{ Conditions for } & \\
Killing vector & Causality/Weyl & \multicolumn{1}{|c|}{ Supersymmetry } & $\nu$ \\
\hline \hline $\boldsymbol{e}_{13}+\boldsymbol{e}_{24}+\varphi_{1} \boldsymbol{e}_{56}+\varphi_{2} \boldsymbol{e}_{78}$ & $\varphi_{1} \geq\left|\varphi_{2}\right| \geq 0$ & $\varphi_{1}+\varphi_{2}=\theta_{1} \pm \theta_{2}$ & $\frac{1}{8}$ \\
$+\theta_{1} R_{12}+\theta_{2} R_{34}$ & $\theta_{1} \geq \theta_{2} \geq 0$ & $\varphi_{1}=-\varphi_{2}, \theta_{1}=\theta_{2} \neq 0$ & $\frac{1}{4}$ \\
& & $\varphi_{1}=-\varphi_{2}, \theta_{1}=\theta_{2}=0$ & $\frac{1}{2}$ \\
\hline $\boldsymbol{e}_{13}-\boldsymbol{e}_{34}+\boldsymbol{e}_{25}-\boldsymbol{e}_{56}$ & $\theta_{1} \geq \theta_{2} \geq 0$ & $\varphi=\theta_{1} \pm \theta_{2}$ & $\frac{1}{8}$ \\
$+\varphi \boldsymbol{e}_{78}+\theta_{1} R_{12}+\theta_{2} R_{34}$ & $\varphi \geq 0$ & $\varphi=0, \theta_{1}=\theta_{2}$ & $\frac{1}{4}$ \\
& & $\varphi=\theta_{1}, \theta_{2}=0$ & $\frac{1}{4}$ \\
& & $\varphi=\theta_{i}=0$ & $\frac{1}{2}$ \\
\hline
\end{tabular}

Table 9: Smooth spacelike supersymmetric quotients of $\mathrm{AdS}_{7} \times S^{4}$

\section{Supersymmetry of singular quotients}

Although in the main body of this work we have focused on the supersymmetry preserved by smooth and everywhere spacelike quotients of FreundRubin backgrounds of the form $\operatorname{AdS}_{p+1} \times S^{q}$, the technology we have developed applies equally to quotients with singularities: either of causal or 
differential/topological type. It is beyond the scope of this work to study in detail the singular quotients of such Freund-Rubin backgrounds. Instead we provide in this section an indication of the conditions under which supersymmetry is preserved in a singular quotient.

A preliminary observation is that it does make sense to talk about spin structure and supersymmetry for a quotient $M / \Gamma$ of a spin manifold $M$ by a group $\Gamma$ acting via orientation-preserving isometries, even if the quotient is singular, at least for the geometries of interest. Indeed, it follows from our discussion in Section 5.1 on the existence of spin structures on the quotient of $M=\operatorname{AdS}_{p+1} \times S^{q}$ by $\Gamma$, that the action of $\Gamma$ on $P_{\mathrm{SO}}(M)$ is free even when the action on $M$ is not. This means that we can define the frame bundle $P_{\mathrm{SO}}(M / \Gamma)$ of a singular quotient simply as $P_{\mathrm{SO}}(M) / \Gamma$. This definition agrees with the standard definition when $M / \Gamma$ is regular. In the same way, provided that the action of $\Gamma$ lifts to the spin bundle $P_{\text {Spin }}(M)$, this action will be free, and we can again define a spin structure on $M / \Gamma$ simply by $P_{\text {Spin }}(M) / \Gamma$, which again agrees with the standard definition if the quotient is regular.

As explained in Section 5.1, if $\Gamma \cong \mathbb{R}$, the quotient will always admit a spin structure, whereas if $\Gamma \cong S^{1}$, there is a possible obstruction which can be tested by examining the weights of $\Gamma$ on the (complexified) Killing spinors. These weights can either be integral or half-integral. If the weights are half-integral there is no spin structure on $M / \Gamma$, but only a $\operatorname{Spin}^{c}$ structure, whereas if the weights are integral, there is a spin structure and moreover the (geometrically realised) supersymmetry corresponds to the zero weights.

In this section we will examine the weights of $\Gamma$, or equivalently, of the Lie algebra element generating $\Gamma$, using the classification of adjoint orbits of $\mathfrak{s o}(2, p) \oplus \mathfrak{s o}(q+1)$ in terms of direct sums of elementary blocks. In Section 7.1 we consider the elementary blocks themselves and in Section 7.2 we consider their direct sums. We shall focus on the existence of zero weights; that is, on the conditions for the preservation of supersymmetry.

\subsection{Elementary blocks}

In this section we will examine the action of each elementary block $B^{m, n} \in$ $\mathfrak{s o}(m, n)$ acting on the spinor representation $\Delta^{m, n}$, or in its complexification. We recall that every element $\boldsymbol{e}_{i j} \in \mathfrak{s o}(m, n)$ acts on $\Delta^{m, n}$ as $\frac{1}{2} \gamma_{i j}$. 


\subsection{1 $\quad B^{(0,2)}(\varphi)$}

This block corresponds to a spatial rotation. It is represented by $\frac{1}{2} \varphi \gamma_{34}$, a semisimple operator with eigenvalue $\frac{i \varphi}{2}$ on $\Delta^{0,2} \cong \mathbb{C}$ and eigenvalue $-\frac{i \varphi}{2}$ on the conjugate representation $\bar{\Delta}^{0,2}$.

\subsection{2 $B^{(1,1)}(\beta)$}

This block corresponds to a boost. It is representated by a semisimple operator $\frac{1}{2} \beta \gamma_{13}$, whose eigenvalues are real and equal to $\pm \frac{\beta}{2}$ on $\Delta_{ \pm}^{1,1} \cong \mathbb{R}$.

\subsection{3 $B^{(2,0)}(\varphi)$}

This block is a timelike rotation. It is representated in by $\frac{1}{2} \varphi \gamma_{12}$, a semisimple operator with imaginary eigenvalues $\frac{i \varphi}{2}$ on $\Delta^{2,0} \cong \mathbb{C}$ and $-\frac{i \varphi}{2}$ on $\bar{\Delta}^{2,0}$.

Quotienting any $\mathrm{AdS}_{p+1} \times S^{q}$ by the action of any one of the above three elementary blocks clearly breaks all supersymmetry.

\subsection{4 $B^{(1,2)}$}

This block is a null rotation. It is represented in $\Delta^{1,2} \cong \mathbb{R}^{2}$ by a nilpotent operator $\frac{1}{2}\left(\gamma_{13}-\gamma_{34}\right)$, which has a one-dimensional kernel.

\subsection{5 $\quad B^{(2,1)}$}

This block is also null rotation, but 'timelike'. It is represented in $\Delta^{2,1} \cong \mathbb{R}^{2}$ by a nilpotent operator $\frac{1}{2}\left(\gamma_{12}-\gamma_{23}\right)$, whose kernel, as in the previous case, is one-dimensional.

In summary the quotients of any $\mathrm{AdS}_{p+1} \times S^{q}$ associated to these two blocks preserve half the supersymmetry.

\subsection{6 $B_{ \pm}^{(2,2)}$}

This block is a 'rotation' in a totally null 2-plane. It is represented in $\Delta^{2,2} \cong$ $\mathbb{R}^{2} \oplus \mathbb{R}^{2}$ as a nilpotent operator $N^{ \pm}=\frac{1}{2}\left(\mp \gamma_{12}-\gamma_{13} \pm \gamma_{24}+\gamma_{34}\right)$ which is itself 
the product of two nilpotent operators $N^{ \pm}=\frac{1}{2} N_{2}^{ \pm} N_{1}=\frac{1}{2}\left( \pm \gamma_{2}+\gamma_{3}\right)\left(\gamma_{1}+\gamma_{4}\right)$. As we saw in Section 6.1.4, the kernel is the subspace generated by the kernels of $N_{1}$ and $N_{2}^{ \pm}$, which is three-dimensional. More precisely, since

$$
N^{ \pm}\left(\nVdash \mp \gamma_{1234}\right)=0 \text {, }
$$

$N^{ \pm}$acts trivially in $\Delta_{\mp}^{2,2} \cong \mathbb{R}^{2}$ and hence has a one-dimensional kernel in $\Delta_{ \pm}^{2,2}$

The supersymmetry preserved by such a quotient depends on whether the Killing spinors belong to $\Delta_{ \pm}^{2,2}$ or to $\Delta^{2,2}$. The quotient preserves all the supersymmetry in $\Delta_{\mp}^{2,2}$ and half the supersymmetry in $\Delta_{ \pm}^{2,2}$ and hence three fourths of the one in $\Delta^{2,2}$.

\subsection{7 $B_{ \pm}^{(2,2)}(\beta)$}

This block is a 'deformation' of the previous one by a selfdual (or antiselfdual) boost. It is represented in $\Delta^{2,2}$ by $N^{ \pm}+S^{ \pm}$, where $N^{ \pm}$is as above and $S^{ \pm}=\frac{1}{2} \beta\left(\gamma_{14} \mp \gamma_{23}\right)$ is semisimple. It is easy to see that $\operatorname{ker} S^{ \pm}=\Delta_{ \pm}^{2,2}$ and that $S^{ \pm}$has eigenvalues $\beta$ and $-\beta$ on $\Delta_{\mp}^{2,2}$. Since $N^{ \pm} S^{ \pm}=S^{ \pm} N^{ \pm}=0$, it follows that the kernel of $N^{ \pm}+S^{ \pm}$is a one-dimensional subspace of $\Delta_{ \pm}^{2,2}$.

The quotient preserves half the supersymmetry in $\Delta_{ \pm}^{2,2}$ and none of the supersymmetry in $\Delta_{\mp}^{2,2}$, whence it preserves one fourth of the one in $\Delta^{2,2}$.

\subsection{8 $B_{ \pm}^{(2,2)}(\varphi)$}

This block is a different deformation of $B_{ \pm}^{(2,2)}$, this time by a selfdual (or anti-selfdual) rotation. It is represented in $\Delta^{2,2}$ by $N^{ \pm}+S^{ \pm}$, where $N^{ \pm}$is as above and $S^{ \pm}=\frac{1}{2} \varphi\left( \pm \gamma_{12}+\gamma_{34}\right)$ is semisimple. It is not hard to see that $N^{ \pm} S^{ \pm}=S^{ \pm} N^{ \pm}=0$ and that $\operatorname{ker} S^{ \pm}=\Delta_{ \pm}^{2,2}$. It follows that the kernel of $N^{ \pm}+S^{ \pm}$is a one dimensional subspace of $\Delta_{ \pm}^{2,2}$.

The quotient preserves half the supersymmetry in $\Delta_{ \pm}^{2,2}$ and none of the supersymmetry in $\Delta_{\mp}^{2,2}$ and hence one fourth of the one in $\Delta^{2,2}$.

\subsection{9 $B_{ \pm}^{(2,2)}(\beta, \varphi)$}

This block consists of a selfdual (resp. anti-selfdual) rotation and a commuting anti-selfdual (resp. selfdual) boost. It is represented in $\Delta^{2,2}$ by the sum 
$S_{1}^{ \pm}+S_{2}^{ \pm}$of two commuting semisimple operators, where $S_{1}^{ \pm}=\frac{1}{2} \varphi\left( \pm \gamma_{12}-\gamma_{34}\right)$ and $S_{2}^{ \pm}=\frac{1}{2} \beta\left(\gamma_{14} \mp \gamma_{23}\right)$. On the complexification of $\Delta^{2,2}, S_{1}^{ \pm}$has pure imaginary eigenvalues whereas $S_{2}^{ \pm}$has real eigenvalues, hence the kernel of their sum is the intersection of their kernels. Since the selfduality properties of $S_{1}^{ \pm}$ and $S_{2}^{ \pm}$are opposite, they leave invariant different subspaces: $\operatorname{ker} S_{1}^{ \pm}=\Delta_{\mp}^{2,2}$ whereas $\operatorname{ker} S_{2}^{ \pm}=\Delta_{ \pm}^{2,2}$. In other words, all supersymmetry is broken in any quotient of $\operatorname{AdS}_{p+1} \times S^{q}$ by the action of this block; although as we will see in the next section, there are decomposable blocks containing $B_{ \pm}^{(2,2)}(\beta, \varphi)$ in which supersymmetry is partially restored.

\subsubsection{0 $B^{(2,3)}$}

The block $B^{2,3}$ is represented in $\Delta^{2,3} \cong \mathbb{R}^{4}$ by the nilpotent operator

$$
N=\frac{1}{2}\left(\gamma_{12}+\gamma_{13}+\gamma_{15}-\gamma_{24}-\gamma_{34}-\gamma_{45}\right)
$$

which can be shown to satisfy $N^{4}=0$. We can rewrite $N$ as

$$
N=\frac{1}{2}\left(\gamma_{1}+\gamma_{4}\right)\left(\gamma_{2}+\gamma_{3}\right)+\frac{1}{2}\left(\gamma_{1}-\gamma_{4}\right) \gamma_{5},
$$

which suggests the following decomposition

$$
\Delta^{2,3}=V \oplus\left(\gamma_{2}+\gamma_{3}\right) V \oplus\left(\gamma_{1}+\gamma_{4}\right) V \oplus\left(\gamma_{2}+\gamma_{3}\right)\left(\gamma_{1}+\gamma_{4}\right) V,
$$

analogous to the one in Section 6.3.6, where now $V=\operatorname{ker}\left(\gamma_{1}-\gamma_{4}\right) \cap \operatorname{ker}\left(\gamma_{2}-\right.$ $\left.\gamma_{3}\right)$. Let $\varepsilon \in \Delta^{2,3}$ be decomposed accordingly as

$$
\varepsilon=\varepsilon_{1}+\left(\gamma_{2}+\gamma_{3}\right) \varepsilon_{2}+\left(\gamma_{1}+\gamma_{4}\right) \varepsilon_{3}+\left(\gamma_{2}+\gamma_{3}\right)\left(\gamma_{1}+\gamma_{4}\right) \varepsilon_{4},
$$

where $\varepsilon_{i} \in V$, whence

$$
N \varepsilon=2 \gamma_{5} \varepsilon_{3}+2\left(\gamma_{2}+\gamma_{3}\right) \gamma_{5} \varepsilon_{4}+\frac{1}{2}\left(\gamma_{1}+\gamma_{4}\right)\left(\gamma_{2}+\gamma_{3}\right) \varepsilon_{1},
$$

where $\gamma_{5} \varepsilon_{i} \in V$. Therefore we see that $N \varepsilon=0$ if and only if $\varepsilon_{1}=\varepsilon_{3}=\varepsilon_{4}=$ 0 . In other words, $\varepsilon=\left(\gamma_{2}+\gamma_{3}\right) \varepsilon_{2}$, and ker $N$ is one-dimensional.

Any quotient of $\operatorname{AdS}_{p+1} \times S^{q}$, for $p \geq 3$, by the action of this block, preserves one fourth of the supersymmetry.

\subsubsection{1 $B_{ \pm}^{(2,4)}(\varphi)$}

This block is a deformation of the double null rotation. It is represented in $\Delta^{2,4} \cong \mathbb{C}^{4}$ by a sum of a nilpotent operator

$$
N^{ \pm}=\frac{1}{2}\left(\gamma_{1}-\gamma_{3}\right) \gamma_{5}+\left( \pm \gamma_{2}-\gamma_{4}\right) \gamma_{6}
$$


which is itself the sum of two commuting nilpotent operators, and a commuting semisimple operator

$$
S^{ \pm}=\frac{1}{2} \varphi\left(\mp \gamma_{12}+\gamma_{34}+\gamma_{56}\right) .
$$

The kernel of $N^{ \pm}+S^{ \pm}$is thus the intersection of the kernels of $N^{ \pm}$and $S^{ \pm}$. However it is easy to see that $S^{ \pm}$has no zero weights in $\Delta^{2,4}$. Therefore this quotient breaks all supersymmetry; although as we will see in the next section, there are decomposable blocks containing $B_{ \pm}^{(2,4)}(\varphi)$ in which supersymmetry is again partially restored.

The supersymmetry fractions preserved by quotients corresponding to elementary blocks is summarised in Table 10. The notation is such that $\nu$ is the fraction of the supersymmetry preserved by a block $B^{m, n}$ on $\Delta^{m, n}$, whereas $\nu_{ \pm}$is the fraction relative to $\Delta_{ \pm}^{m, n}$ whenever this refinement is necessary.

\begin{tabular}{|c|c|c|c|c|}
\hline Block & $\nu$ & Block & $\nu$ & $\left(\nu_{+}, \nu_{-}\right)$ \\
\hline$B^{(0,2)}(\varphi)$ & 0 & $B_{+}^{(2,2)}$ & $\frac{3}{4}$ & $\left(\frac{1}{2}, 1\right)$ \\
\hline$B^{(1,1)}(\beta)$ & 0 & $B_{-}^{(2,2)}$ & $\frac{3}{4}$ & $\left(1, \frac{1}{2}\right)$ \\
\hline$B^{(2,0)}(\varphi)$ & 0 & $B_{+}^{(2,2)}(\beta)$ & $\frac{1}{4}$ & $\left(\frac{1}{2}, 0\right)$ \\
\hline$B^{(1,2)}$ & $\frac{1}{2}$ & $B_{-}^{(2,2)}(\beta)$ & $\frac{1}{4}$ & $\left(0, \frac{1}{2}\right)$ \\
\hline$B^{(2,1)}$ & $\frac{1}{2}$ & $B_{+}^{(2,2)}(\varphi)$ & $\frac{1}{4}$ & $\left(\frac{1}{2}, 0\right)$ \\
\hline$B^{(2,3)}$ & $\frac{1}{4}$ & $B_{-}^{(2,2)}(\varphi)$ & $\frac{1}{4}$ & $\left(0, \frac{1}{2}\right)$ \\
\hline$B_{+}^{(2,4)}(\varphi)$ & 0 & $B_{+}^{(2,2)}(\beta, \varphi)$ & 0 & \\
\hline$B_{-}^{(2,4)}(\varphi)$ & 0 & $B_{-}^{(2,2)}(\beta, \varphi)$ & 0 & \\
\hline
\end{tabular}

Table 10: The supersymmetry preserved by the elementary blocks.

\subsection{Decomposable blocks}

As discussed in detail in the paper, generic quotients of $\operatorname{AdS}_{p+1} \times S^{q}$ are not generated simply by elementary blocks, but by sums of elementary blocks acting on orthogonal subspaces giving rise to an element of $\mathfrak{s o}(2, p) \oplus \mathfrak{s o}(q+1)$. The precise blocks which can occur depend crucially on the parameters $p$ and $q$, of course, as we have seen in Section 4. 
To each decomposable block there is associated an operator $B$ acting on the relevant spinorial representation of $\mathfrak{s o}(2, p) \oplus \mathfrak{s} \mathfrak{o}(q+1)$, in whose kernel (if any) are contained the supersymmetries preserved in the corresponding quotient. As we have seen in Section 6 and indeed in Section 7.1, $B$ decomposes into a sum of commuting operators which can be of three types: nilpotent $(N)$, semisimple with real eigenvalues $\left(S_{R}\right)$ and semisimple with pure imaginary eigenvalues $\left(S_{I}\right)$. In all, there are fourteen different types of decomposable blocks, which we arrange according to the form of $B$. Four cases arise: $N+S_{R}+S_{I}, N+S_{I}, S_{R}+S_{I}$ and $S_{I}$, which will be discussed in turn after the following general remark.

The supersymmetries preserved by the quotient corresponding to $B$ are given by the kernel of $B$ acting on the relevant spinorial representation. It is a standard fact in linear algebra that the kernel of $B=N+S_{R}+S_{I}$ is the intersection of the kernels of $N, S_{R}$ and $S_{I}$. The kernel of $S_{I}$ can be determined by a detailed analysis of the weights of $S_{I}$ in the spinorial representation. This has been done for the smooth spacelike quotients in Section 6 and we will not perform this analysis for the singular quotients in what follows. We will simply point out when preservation of supersymmetry is possible provided that the angles in the expression for $S_{I}$ are suitably fine-tuned.

The results are summarised in Tables 11, 12, 13 and 14, in which the notation $\bigoplus_{i} B^{(0,2)}\left(\varphi_{i}\right)$ denotes a block which can contain sub-blocks acting on the sphere. The second column in the tables contain the corresponding adjoint orbits of $\mathfrak{s o}(2,6)$ following the enumeration in Section 4.4. Some of these adjoint orbits also exist in smaller $\mathfrak{s o}(2, p<6)$.

\subsection{1 $B=N+S_{R}+S_{I}$}

In Table 11 we list the possible decomposable blocks giving rise to a spinor operator of the form $N+S_{R}+S_{I}$.

\begin{tabular}{|l|l|}
\hline \multicolumn{1}{|c|}{ Block } & \multicolumn{1}{c|}{ Cases } \\
\hline \hline$B_{ \pm}^{(2,2)}(\beta) \oplus \bigoplus_{i} B^{(0,2)}\left(\varphi_{i}\right)$ & $7,19,34$ \\
$B^{(1,1)}(\beta) \oplus B^{(1,2)} \oplus \bigoplus_{i} B^{(0,2)}\left(\varphi_{i}\right)$ & 15,29 \\
\hline
\end{tabular}

Table 11: Decomposable blocks of the form $N+S_{R}+S_{I}$

The quotient by $B_{ \pm}^{(2,2)}(\beta) \oplus \bigoplus_{i} B^{(0,2)}\left(\varphi_{i}\right)$ preserves supersymmetry if and 
only if $\bigoplus_{i} B^{(0,2)}\left(\varphi_{i}\right)$ has zero weights in the spinorial representation, whereas the boost in $B^{(1,1)}(\beta) \oplus B^{(1,2)} \oplus \bigoplus_{i} B^{(0,2)}\left(\varphi_{i}\right)$ breaks all the supersymmetry.

\subsection{2 $B=N+S_{I}$}

In Table 12 we list the possible decomposable blocks giving rise to a spinor operator of the form $N+S_{I}$.

\begin{tabular}{|l|l|}
\hline \multicolumn{1}{|c|}{ Block } & \multicolumn{1}{c|}{ Cases } \\
\hline \hline$B^{(1,2)} \oplus \bigoplus_{i} B^{(0,2)}\left(\varphi_{i}\right)$ & $5,17,31$ \\
$B^{(1,2)} \oplus B^{(1,2)} \oplus \bigoplus_{i} B^{(0,2)}\left(\varphi_{i}\right)$ & 23,37 \\
$B^{(2,1)} \oplus \bigoplus_{i} B^{(0,2)}\left(\varphi_{i}\right)$ & $3,14,28$ \\
$B_{ \pm}^{(2,2)} \oplus \bigoplus_{i} B^{(0,2)}\left(\varphi_{i}\right)$ & $6,21,33$ \\
$B_{ \pm}^{(2,2)}(\varphi) \oplus \bigoplus_{i} B^{(0,2)}\left(\varphi_{i}\right)$ & $8,20,35$ \\
$B^{(2,3)} \oplus \bigoplus_{i} B^{(0,2)}\left(\varphi_{i}\right)$ & 13,27 \\
$B_{ \pm}^{(2,4)}(\varphi) \oplus \bigoplus_{i} B^{(0,2)}\left(\varphi_{i}\right)$ & 18,32 \\
\hline
\end{tabular}

Table 12: Decomposable blocks of the form $N+S_{I}$

The blocks $B^{(1,2)} \oplus \bigoplus_{i} B^{(0,2)}\left(\varphi_{i}\right), B^{(1,2)} \oplus B^{(1,2)} \oplus \bigoplus_{i} B^{(0,2)}\left(\varphi_{i}\right)$ and $B^{(2,1)} \oplus \bigoplus_{i} B^{(0,2)}\left(\varphi_{i}\right)$ preserve one half of the supersymmetry preserved by $\bigoplus_{i} B^{(0,2)}\left(\varphi_{i}\right)$, if any.

The blocks $B_{ \pm}^{(2,2)} \oplus \bigoplus_{i} B^{(0,2)}\left(\varphi_{i}\right)$ and $B_{ \pm}^{(2,2)}(\varphi) \oplus \bigoplus_{i} B^{(0,2)}\left(\varphi_{i}\right)$ preserve supersymmetry if and only if $\bigoplus_{i} B^{(0,2)}\left(\varphi_{i}\right)$ does. The precise fraction depends on the particular spinorial representations to which the Killing spinors belong.

The block $B^{(2,3)} \oplus \bigoplus_{i} B^{(0,2)}\left(\varphi_{i}\right)$ preserves one fourth of the supersymmetry preserved by $\bigoplus_{i} B^{(0,2)}\left(\varphi_{i}\right)$, if any.

Finally, the block $B_{ \pm}^{(2,4)}(\varphi) \oplus \bigoplus_{i} B^{(0,2)}\left(\varphi_{i}\right)$ deserves special attention. Although the elementary block $B_{ \pm}^{(2,4)}(\varphi)$ breaks all the supersymmetry, it is possible to restore supersymmetry by adding $\bigoplus_{i} B^{(0,2)}\left(\varphi_{i}\right)$ and fine-tuning the parameters $\varphi$ and $\varphi_{i}$ in such a way that the corresponding semisimple operator $S_{I}\left(\varphi, \varphi_{i}\right)$ have zero weights in the relevant spinor representation. 


\subsection{3 $B=S_{R}+S_{I}$}

In Table 13 we list the possible decomposable blocks giving rise to a spinor operator of the form $S_{R}+S_{I}$.

\begin{tabular}{|l|l|}
\hline \multicolumn{1}{|c|}{ Block } & \multicolumn{1}{c|}{ Cases } \\
\hline \hline$B^{(1,1)}(\beta) \oplus \bigoplus_{i} B^{(0,2)}\left(\varphi_{i}\right)$ & $2,12,26$ \\
$B^{(1,1)}\left(\beta_{1}\right) \oplus B^{(1,1)}\left(\beta_{2}\right) \oplus \bigoplus_{i} B^{(0,2)}\left(\varphi_{i}\right)$ & $11,25,39$ \\
$B_{ \pm}^{(2,2)}(\beta, \varphi) \oplus \bigoplus_{i} B^{(0,2)}\left(\varphi_{i}\right)$ & $9,22,36$ \\
\hline
\end{tabular}

Table 13: Decomposable blocks of the form $S_{R}+S_{I}$

The boost in $B^{(1,1)}(\beta) \oplus \bigoplus_{i} B^{(0,2)}\left(\varphi_{i}\right)$ breaks all supersymmetry and so do the boosts in $B^{(1,1)}\left(\beta_{1}\right) \oplus B^{(1,1)}\left(\beta_{2}\right) \oplus \bigoplus_{i} B^{(0,2)}\left(\varphi_{i}\right)$, unless $\beta_{1}=\beta_{2}$, in which case this block preserves one half of the supersymmetry preserved by $\bigoplus_{i} B^{(0,2)}\left(\varphi_{i}\right)$, if any.

Finally, there is the possibility of restoration of supersymmetry in the block $B_{ \pm}^{(2,2)}(\beta, \varphi) \oplus \bigoplus_{i} B^{(0,2)}\left(\varphi_{i}\right)$, provided that the angles $\varphi, \varphi_{i}$ belong to the loci where $S_{I}\left(\varphi, \varphi_{i}\right)$ has zero weights. In this case, the block preserves one half of the supersymmetry preserved by $S_{I}$.

\subsection{4 $B=S_{I}$}

In Table 14 we list the possible decomposable blocks giving rise to a spinor operator of the form $S_{I}$.

\begin{tabular}{|l|l|}
\hline \multicolumn{1}{|c|}{ Block } & \multicolumn{1}{c|}{ Cases } \\
\hline \hline$\bigoplus_{i} B^{(0,2)}\left(\varphi_{i}\right)$ & $4,16,30$ \\
$B^{(2,0)}(\varphi) \oplus \bigoplus_{i} B^{(0,2)}\left(\varphi_{i}\right)$ & $1,10,24,38$ \\
\hline
\end{tabular}

Table 14: Decomposable blocks of the form $S_{I}$

In both of these cases, the supersymmetry preserved by the quotient is dictated by the zero weights of the corresponding spinor operator.

It should be remarked that in the case of $\bigoplus_{i} B^{(0,2)}\left(\varphi_{i}\right)$, the quotient will 
not be Hausdorff unless the orbits of the Killing vector are periodic, which requires the angles $\varphi_{i}$ to be rationally related.

\section{Acknowledgments}

We would like to acknowledge useful conversations with Charles Frances, Chris Hull, Takashi Kimura, Felipe Leitner, Andrei Moroianu and Elmer Rees. We are also grateful to Owen Madden and Simon Ross for making the results of their paper [53] known to us prior to publication.

This work and [41] conclude a project conceived while the authors were participating in the programme Mathematical Aspects of String Theory which took place in the Fall of 2001 at the Erwin Schrödinger Institute in Vienna, and it is again our pleasure to thank them for support and for providing such a stimulating environment in which to do research. JMF's participation in that programme was made possible in part by a travel grant from the UK PPARC. JMF would like to acknowledge the support and hospitality of the Weizmann Institute and CERN which he visited during the time that it took to complete and write up this work. In particular he would like to thank Micha Berkooz for the invitation to visit the Weizmann Institute. JMF is a member of EDGE, Research Training Network HPRN-CT-2000-00101, supported by The European Human Potential Programme, and his research is partially supported by the UK EPSRC grant GR/R62694/01. JS would like to thank the School of Mathematics of the University of Edinburgh and the Aspen Center for Physics for kind hospitality during the initial and intermediate stages, respectively, of this work. During the time it took to complete this work, JS was supported by a Marie Curie Fellowship of the European Community programme 'Improving the Human Research Potential and the Socio-Economic Knowledge Base' under the contract number HPMF-CT-2000-00480, by grants from the United States-Israel Binational Science Foundation (BSF), the European RTN network HPRN-CT-200000122, Minerva, the Phil Zacharia fellowship, and by the United States Department of Energy under the grant number DE-FG02-95ER40893 and the National Science Foundation under Grant No. PHY99-07949.

\section{References}

[1] J. M. Figueroa-O'Farrill and J. Simón, "Generalised supersymmetric fluxbranes," J. High Energy Phys. 12 (2001) 011. arXiv:hep-th/0110170. 
[2] M. A. Melvin, "Pure magnetic and electric geons," Phys. Lett. 8 (1964) 65-68.

[3] G. W. Gibbons and D. L. Wiltshire, "Spacetime as a membrane in higher dimensions," Nucl. Phys. B287 (1987) 717.

arXiv:hep-th/0109093.

[4] G. W. Gibbons and K. Maeda, "Black holes and membranes in higher-dimensional theories with dilaton fields," Nucl. Phys. B298 (1988) 741-775.

[5] F. Dowker, J. P. Gauntlett, S. B. Giddings, and G. T. Horowitz, "On pair creation of extremal black holes and Kaluza-Klein monopoles," Phys. Rev. D50 (1994) 2662-2679. arXiv:hep-th/9312172.

[6] F. Dowker, J. P. Gauntlett, G. W. Gibbons, and G. T. Horowitz, "The decay of magnetic fields in Kaluza-Klein theory," Phys. Rev. D52 (1995) 6929-6940. arXiv:hep-th/9507143.

[7] F. Dowker, J. P. Gauntlett, G. W. Gibbons, and G. T. Horowitz, "Nucleation of $p$-branes and fundamental strings," Phys. Rev. D53 (1996) 7115-7128. arXiv:hep-th/9512154.

[8] C. M. Chen, D. V. Gal'tsov, and S. A. Sharakin, "Intersecting M-fluxbranes," Grav. Cosmol. 5 (1999) 45-48. arXiv:hep-th/9908132.

[9] M. S. Costa and M. Gutperle, "The Kaluza-Klein Melvin solution in M-theory," J. High Energy Phys. 03 (2001) 027. arXiv:hep-th/0012072.

[10] M. Gutperle and A. Strominger, "Fluxbranes in string theory," J. High Energy Phys. 06 (2001) 035. arXiv:hep-th/0104136.

[11] P. M. Saffin, "Gravitating fluxbranes," Phys. Rev. D64 (2001) 024014. arXiv:gr-qc/0104014.

[12] M. S. Costa, C. Herdeiro, and L. Cornalba, "Fluxbranes and the dielectric effect in string theory," Nucl. Phys. B619 (2001) 155-190. arXiv:hep-th/0105023.

[13] R. Emparan, "Tubular branes in fluxbranes," Nucl. Phys. B610 (2001) 169-189. arXiv:hep-th/0105062.

[14] D. Brecher and P. M. Saffin, "A note on the supergravity description of dielectric branes," Nucl. Phys. B613 (2001) 218-236. arXiv:hep-th/0106206. 
[15] A. M. Uranga, "Wrapped fluxbranes." arXiv:hep-th/0108196.

[16] R. Emparan and M. Gutperle, "From $p$-branes to fluxbranes and back," J. High Energy Phys. 12 (2001) 023. arXiv:hep-th/0111177.

[17] J. G. Russo and A. A. Tseytlin, "Supersymmetric fluxbrane intersections and closed string tachyons," JHEP 11 (2001) 065. arXiv:hep-th/0110107.

[18] J. Simón, "The geometry of null rotation identifications," J. High Energy Phys. 06 (2002) 001. arXiv:hep-th/0203201.

[19] H. Liu, G. Moore, and N. Seiberg, "Strings in a time-dependent orbifold," J. High Energy Phys. 06 (2002) 045.

arXiv:hep-th/0204168.

[20] H. Liu, G. Moore, and N. Seiberg, "Strings in time-dependent orbifolds," J. High Energy Phys. 10 (2002) 031. arXiv:hep-th/0206182.

[21] M. Fabinger and J. McGreevy, "On smooth time-dependent orbifolds and null singularities," J. High Energy Phys. 06 (2003) 042. arXiv:hep-th/0206196.

$[22]$ G. T. Horowitz and J. Polchinski, "Instability of spacelike and null orbifold singularities," Phys. Rev. D66 (2002) 103512. arXiv:hep-th/0206228.

[23] G. T. Horowitz and A. R. Steif, "Singular string solutions with nonsingular initial data," Phys. Lett. B258 (1991) 91.

[24] J. M. Figueroa-O'Farrill and J. Simón, "Supersymmetric Kaluza-Klein reductions of M2 and M5-branes," Adv. Theor. Math. Phys. 6 (2002) 703-793. arXiv:hep-th/0208107.

[25] J. M. Figueroa-O'Farrill and J. Simón, "Supersymmetric Kaluza-Klein reductions of M-waves and MKK-monopoles," Class. Quant. Grav. 19 (2002) 6147-6174. (Erratum: ibid. 21 (2004) 337) arXiv:hep-th/0208108.

[26] L. Maoz and J. Simón, "Killing spectroscopy of closed timelike curves,". arXiv:hep-th/0310255.

[27] J. M. Figueroa-O'Farrill, H. Rajaniemi, and J. Simón, "Supersymmetric Kaluza-Klein reductions of intersecting M-branes." in preparation. 
[28] J. M. Maldacena, "The large $N$ limit of superconformal field theories and supergravity," Adv. Theor. Math. Phys. 2 (1998) 231-252.

arXiv:hep-th/9711200.

[29] A. Bergman and O. J. Ganor, "Dipoles, twists and noncommutative gauge theory," J. High Energy Phys. 10 (2000) 018. arXiv:hep-th/0008030.

[30] K. Dasgupta, O. J. Ganor, and G. Rajesh, "Vector deformations of $N=4$ super-Yang-Mills theory, pinned branes, and arched strings," $J$. High Energy Phys. 04 (2001) 034. arXiv:hep-th/0010072.

[31] A. Bergman, K. Dasgupta, O. J. Ganor, J. L. Karczmarek, and G. Rajesh, "Nonlocal field theories and their gravity duals," Phys. Rev. D65 (2002) 066005. arXiv:hep-th/0103090.

[32] K. Dasgupta and M. M. Sheikh-Jabbari, "Noncommutative dipole field theories," J. High Energy Phys. 02 (2002) 002. arXiv:hep-th/0112064.

[33] M. Alishahiha and H. Yavartanoo, "Supergravity description of the large N noncommutative dipole field theories," J. High Energy Phys. 04 (2002) 031. arXiv:hep-th/0202131.

[34] R.-G. Cai, J.-X. Lu, and N. Ohta, "NCOS and D-branes in time-dependent backgrounds," Phys. Lett. B551 (2003) 178-186. arXiv:hep-th/0210206.

[35] A. Hashimoto and S. Sethi, "Holography and string dynamics in time-dependent backgrounds," Phys. Rev. Lett. 89 (2002) 261601. arXiv:hep-th/0208126.

[36] J. Simón, "Null orbifolds in AdS, time dependence and holography," JHEP 10 (2002) 036. arXiv:hep-th/0208165.

[37] M. Bañados, C. Teitelboim, and J. Zanelli, "The black hole in three-dimensional spacetime," Phys. Rev. Lett. 69 (1992) 1849.

[38] M. Bañados, M. Henneaux, C. Teitelboim, and J. Zanelli, "Geometry of the 2+1 black hole," Phys. Rev. D48 (1993) 1506.

arXiv:gr-qc: 9302012.

[39] O. Coussaert and M. Henneaux, "Self-dual solutions of $2+1$ einstein gravity with a negative cosmological constant."

arXiv:hep-th/9407181. 
[40] V. Balasubramanian, A. Naqvi, and J. Simón, "A multi-boundary AdS orbifold and DLCQ holography: a universal holographic description of extremal black hole horizons." arXiv:hep-th/0311237.

[41] J. M. Figueroa-O'Farrill, O. Madden, S. F. Ross, and J. Simón, "Quotients of $\operatorname{AdS}_{p+1} \times S^{q}$ : causally well-behaved spaces and black holes." arXiv:hep-th/0402094.

[42] G. T. Horowitz and D. Marolf, "A new approach to string cosmology," JHEP 07 (1998) 014. arXiv:hep-th/9805207.

[43] K. Behrndt and D. Lüst, "Branes, waves and AdS orbifolds," JHEP 07 (1999) 019. arXiv:hep-th/9905180.

[44] B. Ghosh and S. Mukhi, "Killing spinors and supersymmetric AdS orbifolds," JHEP 10 (1999) 021. arXiv:hep-th/9908192.

[45] R.-G. Cai, "Constant curvature black hole and dual field theory," Phys. Lett. B544 (2002) 176-182. arXiv:hep-th/0206223.

[46] P. Bieliavsky, S. Detournay, M. Herquet, M. Rooman, and P. Spindel, "Global geometry of the $2+1$ rotating black hole," Phys. Lett. B570 (2003) 231-236. arXiv: hep-th/0306293.

[47] P. Bieliavsky, M. Rooman, and P. Spindel, "Regular poisson structures on massive non-rotating btz black holes," Nucl. Phys. B645 (2002) 349-364. arXiv:hep-th/0206189.

[48] B. McInnes, "Orbifold physics and de sitter spacetime." arXiv:hep-th/0311055.

[49] B. McInnes, "String theory and the shape of the universe." arXiv:hep-th/0401035.

[50] B. Fiol, C. Hofman, and E. Lozano-Tellechea, "Causal structure of $d=5$ vacua and axisymmetric spacetimes." arXiv:hep-th/0312209.

[51] C. Boubel, Sur l'holonomie des variétés pseudo-riemanniennes. PhD thesis, Université Henri Poincaré, Nancy I, 2000.

[52] S. Holst and P. Peldán, "Black holes and causal structure in anti-de Sitter isometric spacetimes," Class. Quant. Grav. 14 (1997) 3433-3452. arXiv:gr-qc/9705067.

[53] O. Madden and S. F. Ross, "Quotients of anti-de sitter space." arXiv:hep-th/0401205. 
[54] C. Bär, "Real Killing spinors and holonomy," Comm. Math. Phys. 154 (1993) 509-521.

[55] I. Kath, "Killing spinors on pseudo-riemannian manifolds," 1999. Habilitationsschrift, Humboldt-Universität zu Berlin.

[56] J. M. Figueroa-O'Farrill, F. Leitner, and J. Simón, "Supersymmetric Freund-Rubin backgrounds." in preparation.

[57] J. M. Figueroa-O'Farrill, "On the supersymmetries of Anti-de Sitter vacua," Class. Quant. Grav. 16 (1999) 2043-2055.

arXiv:hep-th/9902066.

[58] T. Neukirchner, "Normalform eines schief- oder selbstadjungierten Endomorphismus auf einem pseudo-euklidischen oder symplektischen Vektorraum über $\mathbb{R}$ oder $\mathbb{C}$ - eine Arbeit von Charles Boubel." Humboldt Universität preprint.

[59] F. Leitner, "Imaginary Killing spinors in lorentzian geometry." arXiv:math.DG/0302024.

[60] M. J. Duff, H. Lü, and C. N. Pope, "Supersymmetry without supersymmetry," Phys. Lett. B409 (1997) 136-144. arXiv:hep-th/9704186.

[61] M. J. Duff, H. Lü, and C. N. Pope, "AdS ${ }_{5} \times S^{5}$ untwisted," Nucl. Phys. B532 (1998) 181-209. arXiv:hep-th/9803061.

[62] C. N. Pope, A. Sadrzadeh, and S. R. Scuro, "Timelike Hopf duality and type IIA* string solutions," Class. Quant. Grav. 17 (2000) 623-641. arXiv:hep-th/9905161.

[63] C. M. Hull, "Holonomy and symmetry in M-theory." arXiv:hep-th/0305039.

[64] A. Moroianu, Opérateur de Dirac et submersions riemanniennes. $\mathrm{PhD}$ thesis, École Polytechnique, Palaiseau, 1996.

[65] B. Ammann and C. Bär, "The Dirac operator on nilmanifolds and collapsing circle bundles," Ann. Global Anal. Geom. 16 (1998) $221-253$.

[66] Y. Kosmann, "Dérivées de Lie des spineurs," Annali di Mat. Pura Appl. (IV) 91 (1972) 317-395.

[67] A. H. Chamseddine, J. M. Figueroa-O'Farrill, and W. A. Sabra, "Vacuum solutions of six-dimensional supergravities and lorentzian Lie groups." arXiv:hep-th/0306278. 
[68] J. P. Gauntlett, R. C. Myers, and P. K. Townsend, "Supersymmetry of rotating branes," Phys. Rev. D59 (1999) 025001.

arXiv:hep-th/9809065.

[69] J. C. Breckenridge, R. C. Myers, A. W. Peet, and C. Vafa, "D-branes and spinning black holes," Phys. Lett. B391 (1997) 93-98.

arXiv:hep-th/9602065.

[70] R. Kallosh, A. Rajaraman, and W. K. Wong, "Supersymmetric rotating black holes and attractors," Phys. Rev. D55 (1997) 3246. arXiv:hep-th/9611094.

[71] J. E. Humphreys, Introduction to Lie algebras and representation theory, vol. 9 of Graduate Texts in Mathematics. Springer-Verlag, New York, 1978. Second printing, revised.

[72] J. Molins and J. Simón, "BPS states and automorphisms," Phys. Rev. D62 (2000) 125019. arXiv:hep-th/0007253.

[73] J. P. Gauntlett and C. M. Hull, "BPS states with extra supersymmetry," JHEP 01 (2000) 004. arXiv:hep-th/9909098. 